BULLETIN (New Series) OF THE

AMERICAN MATHEMATICAL SOCIETY

Volume 47, Number 3, July 2010, Pages 483-552

S 0273-0979(10)01294-2

Article electronically published on March 11, 2010

\title{
THE ALGEBRA OF GRAND UNIFIED THEORIES
}

\author{
JOHN BAEZ AND JOHN HUERTA
}

\begin{abstract}
The Standard Model is the best tested and most widely accepted theory of elementary particles we have today. It may seem complicated and arbitrary, but it has hidden patterns that are revealed by the relationship between three "grand unified theories": theories that unify forces and particles by extending the Standard Model symmetry group $\mathrm{U}(1) \times \mathrm{SU}(2) \times \mathrm{SU}(3)$ to a larger group. These three are Georgi and Glashow's SU(5) theory, Georgi's theory based on the group Spin(10), and the Pati-Salam model based on the group $\mathrm{SU}(2) \times \mathrm{SU}(2) \times \mathrm{SU}(4)$. In this expository account for mathematicians, we explain only the portion of these theories that involves finite-dimensional group representations. This allows us to reduce the prerequisites to a bare minimum while still giving a taste of the profound puzzles that physicists are struggling to solve.
\end{abstract}

\section{INTRODUCTION}

The Standard Model of particle physics is one of the greatest triumphs of physics. This theory is our best attempt to describe all the particles and all the forces of nature. . except gravity. It does a great job of fitting experiments we can do in the lab. But physicists are dissatisfied with it. There are three main reasons. First, it leaves out gravity: that force is described by Einstein's theory of general relativity, which has not yet been reconciled with the Standard Model. Second, astronomical observations suggest that there may be forms of matter not covered by the Standard Model-most notably, "dark matter". Third, the Standard Model is complicated and seemingly arbitrary. This goes against the cherished notion that the laws of nature, when deeply understood, are simple and beautiful.

For the modern theoretical physicist, looking beyond the Standard Model has been an endeavor both exciting and frustrating. Most modern attempts are based on string theory. There are also other interesting approaches, such as loop quantum gravity and theories based on noncommutative geometry. But back in the mid 1970 s, before any of the currently popular approaches rose to prominence, physicists pursued a program called "grand unification". This sought to unify the forces and particles of the Standard Model using the mathematics of Lie groups, Lie algebras, and their representations. Ideas from this era remain influential, because grand unification is still one of the most fascinating attempts to find order and beauty lurking within the Standard Model.

Received by the editors May 8, 2009, and, in revised form, October 16, 2009.

2000 Mathematics Subject Classification. Primary 20C35, 81R05; Secondary 81-02.

Key words and phrases. Grand unified theory, standard model, representation theory.

This research was supported by a grant from the Foundational Questions Institute. 
This paper is a gentle introduction to the group representations that describe particles in the Standard Model and the most famous grand unified theories. To make the material more approachable for mathematicians, we limit our scope by not discussing particle interactions or "symmetry breaking", i.e., the way a theory with a large symmetry group can mimic one with a smaller group at low energies. These topics lie at the very heart of particle physics. But by omitting them, we can focus on ideas from algebra that many mathematicians will find familiar, while introducing the unfamiliar ways that physicists use these ideas.

In fact, the essential simplicity of the representation theory involved in the Standard Model and grand unified theories is quite striking. The usual textbook approach to particle physics proceeds through quantum field theory and gauge theory. While these subjects are very important to modern mathematics, learning them is a major undertaking. We have chosen to focus on the algebra of grand unified theories because many mathematicians have the prerequisites to understand it with only a little work.

A full-fledged treatment of particle physics requires quantum field theory, which uses representations of a noncompact Lie group called the Poincaré group on infinite-dimensional Hilbert spaces. This brings in a lot of analytical subtleties, which make it hard to formulate theories of particle physics in a mathematically rigorous way. In fact, no one has yet succeeded in doing this for the Standard Model. But by neglecting the all-important topic of particle interactions, we can restrict attention to finite-dimensional Hilbert spaces: that is, finite-dimensional complex inner product spaces. This makes our discussion purely algebraic in flavor.

Every theory we consider has an "internal symmetry group" or "gauge group". This is a compact Lie group, say $G$. Particles then live in representations of $G$ on a finite-dimensional Hilbert space $V$. More precisely: $V$ can always be decomposed as a direct sum of irreducible representations, or irreps, and for our limited purposes, particles are basis vectors of irreps. This provides a way to organize particles, which physicists have been exploiting since the 1960s.

The idea of "unification" has a clear meaning in these terms. Suppose $V$ is a representation, not only of $G$, but also of some larger group $H$ having $G$ as a subgroup. Then we expect $V$ to decompose into fewer irreps as a representation of $H$ than as a representation of $G$, because elements of $H$ can mix different irreps of $G$. So, by introducing a larger symmetry group, particles can be unified into larger irreps.

"Grand unification" occurs when the compact Lie group $G$ is simple, and thus not a product of other groups. A gauge theory based on $G$ requires an invariant inner product on its Lie algebra. When $G$ is simple, this form is unique up to a scale factor, which physicists call a "coupling constant": this measures the strength of the force corresponding to $G$. When $G$ is the product of simple factors, there is one coupling constant for each factor of $G$. So, by using a simple Lie group as a gauge group, we minimize the number of coupling constants.

In this paper, we give an account of the algebra behind the Standard Model and three attempts at unification: Georgi and Glashow's SU(5) theory, Georgi's theory based on the group Spin(10) (physicists call this the $\mathrm{SO}(10)$ theory), and the Pati-Salam model. All three date to around 1974. The first two are known as grand unified theories, or GUTs, because they are based on simple Lie groups. The Pati-Salam model is different: while it is called a GUT by some authors, and 
does indeed involve unification, it is based on the Lie group $\mathrm{SU}(2) \times \mathrm{SU}(2) \times \mathrm{SU}(4)$, which is merely semisimple.

It is important to note that these theories have their problems. The $\mathrm{SU}(5)$ theory predicts that protons will decay more quickly than they do, and it requires certain trends to hold among the relative strengths of forces at high energies - trends which the data do not support. The $\mathrm{SO}(10)$ theory may still be viable, especially if at low enough energies it reduces to the Pati-Salam model. However, the issues involved are complex. For details, see the paper by Bertolini et al. and the many references therein [4].

Nonetheless, it is still very much worthwhile for mathematicians to study the algebra of grand unified theories. First, even apart from their physical significance, these theories are intrinsically beautiful mathematical structures. Second, they provide a nice way for mathematicians to get some sense of the jigsaw puzzle that physicists are struggling to solve. It is certainly hopeless trying to understand what physicists are trying to accomplish with string theory without taking a look at grand unified theories. Finally, grand unified theories can be generalized by adding "supersymmetry", and the resulting generalizations are considered serious contenders for describing the real world. For some recent overviews of their prospects, see Pati [26, 27] and Peskin 29].

This is how we shall proceed. In Section 2 we start by describing the Standard Model. After a brief nod to the electron and photon, we explain some nuclear physics in Section 2.1. We start with Heisenberg's old attempt to think of the proton and neutron as two states of a single particle, the "nucleon", described by a two-dimensional representation of $\mathrm{SU}(2)$. The idea of unification through representation theory traces its origins back to this notion.

After this warmup we tour the Standard Model in its current form. In Section 2.2 we describe the particles called "fundamental fermions", which constitute matter. In Section 2.3 we describe the particles called "gauge bosons", which carry forces. Apart from the elusive Higgs boson, all particles in the Standard Model are of these two kinds. In Section 2.4 we give a more mathematical treatment of these ideas: the gauge bosons are determined by the Standard Model gauge group

$$
G_{\mathrm{SM}}=\mathrm{U}(1) \times \mathrm{SU}(2) \times \mathrm{SU}(3),
$$

while the fundamental fermions and their antiparticles are basis vectors of a highly reducible representation of this group, which we call $F \oplus F^{*}$. Here $F$ describes the fermions, while $F^{*}$ describes their antiparticles.

Amazingly, using the ideas of gauge theory and quantum field theory, plus the "Higgs mechanism" for symmetry breaking, we can recover the dynamical laws obeyed by these particles from the representation of $G_{\mathrm{SM}}$ on $F \oplus F^{*}$. This information is enough to decode the physics of these particles and make predictions about what is seen in the gigantic accelerators that experimental physicists use to probe the natural world at high energies. Unfortunately, to explain all this would go far beyond the modest goals of this paper. For a guide to further study, see Section 1.1.

Having acquainted the reader with the Standard Model of particle physics in Section 2, we then go on to talk about grand unified theories in Section 3. These theories go beyond the Standard Model by "extending" the gauge group. That is, we pick a way to include $G_{\mathrm{SM}}$ in some larger group $G$ and choose a representation $V$ of $G$ which reduces to the Standard Model representation $F \oplus F^{*}$ when we restrict 
it to $G_{\mathrm{SM}}$. We describe how this works for the $\mathrm{SU}(5)$ theory (Section 3.1), the $\mathrm{SO}(10)$ theory (Section 3.2), and the Pati-Salam model (Section 3.3). Of course, since we do not discuss the dynamics, a lot will go unsaid about these GUTs.

As we proceed, we explain how the SU(5) theory and the Pati-Salam model are based on two distinct visions about how to extend the Standard Model. However, we will see that the $\mathrm{SO}(10)$ theory is an extension of both the $\mathrm{SU}(5)$ theory (Section 3.2) and the Pati-Salam model (Section 3.4). Moreover, these two routes to the $\mathrm{SO}(10)$ theory are compatible in a precise sense: we get a commuting square of groups, and a commuting square of representations, which fit together to form a commuting cube (Section 3.5).

In Section 4, we conclude by discussing what this means for physics: namely, how the Standard Model reconciles the two visions of physics lying behind the SU(5) theory and the Pati-Salam model. In a sense, it is the intersection of the SU(5) theory and the Pati-Salam model within their common unification, $\mathrm{SO}(10)$.

Throughout the course of the paper, we occasionally summarize our progress in theorems, most phrased in terms of commutative diagrams:

- Section 3.1, Theorem 1 the SU(5) theory extends the Standard Model.

- Section 3.2, Theorem 2 the Spin(10) theory extends the SU(5) theory.

- Section 3.3, Theorem3 the Pati-Salam model extends the Standard Model.

- Section 3.4, Theorem 4 the Pati-Salam model is isomorphic to a theory involving $\operatorname{Spin}(4) \times \operatorname{Spin}(6)$.

- Section 3.4, Theorem 5 the $\operatorname{Spin}(4) \times \operatorname{Spin}(6)$ theory extends the Standard Model.

- Section 3.4, Theorem [6 the $\operatorname{Spin}(10)$ theory extends the $\operatorname{Spin}(4) \times \operatorname{Spin}(6)$ theory.

- Section 3.5, Theorem7 the Standard Model, the SU(5) theory, the Spin(4) $\times \operatorname{Spin}(6)$ theory and the $\operatorname{Spin}(10)$ theory fit together in a commutative cube.

- Section 4, Theorem 8 The true gauge group of the Standard Model is the intersection of $\mathrm{SU}(5)$ and $\mathrm{SO}(4) \times \mathrm{SO}(6)$ in $\mathrm{SO}(10)$.

- Section 4, Theorem 9, The true gauge group of the Standard Model is the intersection of $\mathrm{SU}(5)$ and $(\operatorname{Spin}(4) \times \operatorname{Spin}(6)) / \mathbb{Z}_{2}$ in $\operatorname{Spin}(10)$.

1.1. Guide to further reading. We have tried to limit our prerequisites to the bare minimum. This includes basic facts about Lie groups, Lie algebras, and their representations, especially finite-dimensional unitary representations of compact Lie groups. We will not need the structure theory for simple Lie groups. We do, however, assume a little familiarity with the classical Lie groups $\operatorname{GL}(n), \operatorname{SL}(n)$, $\mathrm{O}(n), \mathrm{SO}(n), \mathrm{U}(n)$, and $\mathrm{SU}(n)$, as well as their Lie algebras.

There are countless books on Lie groups, Lie algebras and their representations, but the text by Hall 14 has everything we need, and more. Sternberg's introduction to group theory and physics 34 includes an excellent account of applications to particle physics. To see the subject more through the eyes of a physicist, try the books by Lipkin [20] or Tinkham [38. Georgi's text [11] shows how the subject looks to one of the inventors of grand unified theories.

Starting in Section 3.1 we assume familiarity with exterior algebras, and in Section 3.2 we also use Clifford algebras. For what we need, Chevalley's book [6] is more than sufficient. 
For the interested reader, there are many introductions to particle physics where one can learn the dynamics after getting a taste of the algebra here. It might be good to start by reading Griffiths' introductory book [13. together with Sudbery's text specially designed for mathematicians [36]. The book by Huang [17] delves as deep as one can go into the Standard Model without a heavy dose of quantum field theory, and the book by Lee [19] is full of practical wisdom. For more information on grand unified theories, see the textbooks by Ross [31] and Mohapatra [21.

Particle physics relies heavily on quantum field theory. There are many books on this subject, none of which make it easy. Prerequisites include a good understanding of classical mechanics, classical field theory and quantum mechanics. Many physicists consider the books by Brown [5] and Ryder [32] to be the most approachable. The text by Peskin and Schroeder [30] offers a lot of physical insight, and we have also found Zee's book [40] very useful in this respect. Srednicki's text 35] is clear about many details that other books gloss over, and it costs nothing! Of course, these books are geared toward physicists: mathematicians may find the lack of rigor frustrating. Ticciati [37] provides a nice introduction for mathematicians, but anyone serious about this subject should quickly accept the fact that quantum field theory has not been made rigorous: this is a project for the century to come.

Particle physics also relies heavily on geometry, especially gauge theory. This subject is easier to develop in a rigorous way, so there are plenty of texts that describe the applications to physics, but which a mathematician can easily understand. Naber's books are a great place to start [22, 23], and one of us has also written an elementary introduction [3]. Isham's text is elegant and concise [18], and many people swear by Nakahara 24. The quantum field theory texts mentioned above also discuss gauge theory, but in language less familiar to mathematicians.

Finally, few things are more enjoyable than the history of nuclear and particle physics, a romantic tale full of heroic figures and tightly linked to the dark drama of World War II, the Manhattan Project, and the ensuing Cold War. Crease and Mann [8] give a very readable introduction. To dig deeper, try the book by Segrè [33, or the still more detailed treatments by Pais [25] and Hoddeson et al. [16].

\section{The StAndard MOdel}

Today, most educated people know that the world is made of atoms, and that atoms, in turn, are made of electrons, protons, and neutrons. The electrons orbit a dense nucleus made of protons and neutrons, and as the outermost layer of any atom's structure, they are responsible for all chemistry. They are held close to the nucleus by electromagnetic forces: the electrons carry a negative electric charge, and protons carry a positive charge. Opposite charges attract, and this keeps the electrons and the nucleus together.

At one point in time, electrons, protons, and neutrons were all believed to be fundamental and without any constituent parts, just as atoms themselves were once believed to be, before the discovery of the electron. Electrons are the only one of these subatomic particles still considered fundamental, and it is with this venerable particle that we begin a table of the basic constituents of matter, called "fundamental fermions". We will see more soon.

\begin{tabular}{lcc}
\hline \multicolumn{3}{l}{ Fundamental Fermions (first try) } \\
\hline Name & Symbol & Charge \\
\hline Electron & $e^{-}$ & -1 \\
\hline
\end{tabular}


Since the electron is charged, it participates in electromagnetic interactions. From the modern perspective of quantum field theory, electromagnetic interactions are mediated by the exchange of virtual photons, particles of light that we never see in the lab, but whose effects we witness whenever like charges are repelled or opposite charges are attracted. We depict this process with a diagram:

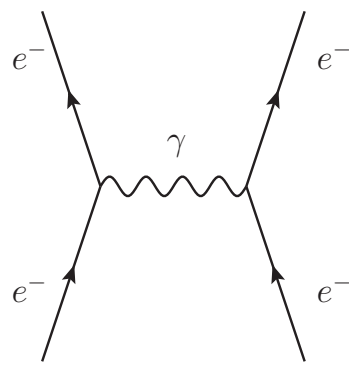

Here, time runs up the page. Two electrons come in, exchange a photon, and leave, slightly repelled from each other by the process.

The photon is our next example of a fundamental particle, though it is of a different character than the electron and quarks. As a mediator of forces, the photon is known as a gauge boson in modern parlance. It is massless and interacts only with charged particles, though it carries no charge itself. So, we begin our list of gauge bosons as follows:

\begin{tabular}{llc}
\hline \multicolumn{3}{c}{ Gauge Bosons (first try) } \\
\hline Force & Gauge Boson & Symbol \\
\hline Electromagnetism & Photon & $\gamma$ \\
\hline
\end{tabular}

2.1. Isospin and $\mathbf{S U}(\mathbf{2})$. Because like charges repel, it is remarkable that the atomic nucleus stays together. After all, the protons are all positively charged and are repelled from each other electrically. To hold these particles so closely together, physicists hypothesized a new force, the strong force, strong enough to overcome the electric repulsion of the protons. It must be strongest only at short distances (about $10^{-15} \mathrm{~m}$ ), and then it must fall off rapidly, for protons are repelled electrically unless their separation is that small. Neutrons must also experience it, because they are bound to the nucleus as well.

Physicists spent several decades trying to understand the strong force; it was one of the principal problems in physics in the mid-twentieth century. About 1932, Werner Heisenberg, pioneer in quantum mechanics, discovered one of the first clues to its nature. He proposed, in [15, that the proton and neutron might really be two states of the same particle, now called the nucleon. In modern terms, he attempted to unify the proton and neutron.

To understand how, we need to know a little quantum mechanics. In quantum mechanics, the state of any physical system is given by a unit vector in a complex Hilbert space, and it is possible to take complex linear combinations of the system in different states. For example, the state for a quantum system, like a particle on a line, is a complex-valued function

$$
\psi \in L^{2}(\mathbb{R}),
$$

or if the particle is confined to a one-dimensional box, so that its position lies in the unit interval $[0,1]$, then its state lives in the Hilbert space $L^{2}([0,1])$. 
We have special rules for combining quantum systems. If, say, we have two particles in a box, particle 1 and particle 2 , then the state is a function of both particle 1's position and particle 2's:

$$
\psi \in L^{2}([0,1] \times[0,1]),
$$

but this is isomorphic to the tensor product of particle 1's Hilbert space with particle 2's:

$$
L^{2}([0,1] \times[0,1]) \cong L^{2}([0,1]) \otimes L^{2}([0,1]) .
$$

This is how we combine systems in general. If a system consists of one part with Hilbert space $V$ and another part with Hilbert space $W$, their tensor product $V \otimes W$ is the Hilbert space of the combined system. Heuristically,

$$
\text { and }=\otimes \text {. }
$$

We just discussed the Hilbert space for two particles in a single box. We now consider the Hilbert space for a single particle in two boxes, by which we mean a particle that is in one box, say $[0,1]$, or in another box, say $[2,3]$. The Hilbert space here is

$$
L^{2}([0,1] \cup[2,3]) \cong L^{2}([0,1]) \oplus L^{2}([2,3]) .
$$

In general, if a system's state can lie in a Hilbert space $V$ or in a Hilbert space $W$, the total Hilbert space is then

$$
V \oplus W
$$

Heuristically,

$$
\text { or }=\oplus \text {. }
$$

Returning to nucleons: according to Heisenberg's theory, a nucleon is a proton or a neutron. If we use the simplest nontrivial Hilbert space for both the proton and neutron, namely $\mathbb{C}$, then the Hilbert space for the nucleon should be

$$
\mathbb{C}^{2} \cong \mathbb{C} \oplus \mathbb{C} .
$$

The proton and neutron then correspond to basis vectors of this Hilbert space:

$$
p=\left(\begin{array}{l}
1 \\
0
\end{array}\right) \in \mathbb{C}^{2}
$$

and

$$
n=\left(\begin{array}{l}
0 \\
1
\end{array}\right) \in \mathbb{C}^{2} .
$$

But, we can also have a nucleon in a linear combination of these states. More precisely, the state of the nucleon can be represented by any unit vector in $\mathbb{C}^{2}$.

The inner product in $\mathbb{C}^{2}$ then allows us to compute probabilities, using the following rule coming from quantum mechanics: the probability that a system in state $\psi \in H$, a given Hilbert space, will be observed in state $\phi \in H$ is

$$
|\langle\psi, \phi\rangle|^{2} \text {. }
$$

Since $p$ and $n$ are orthogonal, there is no chance of seeing a proton as a neutron or vice versa, but for a nucleon in the state

$$
\alpha p+\beta n \in \mathbb{C}^{2},
$$

there is probability $|\alpha|^{2}$ that measurement will result in finding a proton, and $|\beta|^{2}$ that measurement will result in finding a neutron. The condition that our state be a unit vector ensures that these probabilities add to 1 . 
In order for this to be interesting, however, there must be processes that can turn protons and neutrons into different states of the nucleon. Otherwise, there would be no point in having the full $\mathbb{C}^{2}$ space of states. Conversely, if there are processes which can change protons into neutrons and back, it turns out we need all of $\mathbb{C}^{2}$ to describe them.

Heisenberg believed in such processes, because of an analogy between nuclear physics and atomic physics. The analogy turned out to be poor, based on the faulty notion that the neutron was composed of a proton and an electron, but the idea of the nucleon with states in $\mathbb{C}^{2}$ proved to be a breakthrough.

The reason is that in 1936 a paper by Cassen and Condon [7] appeared suggesting that the nucleon's Hilbert space $\mathbb{C}^{2}$ is acted on by the symmetry group $\mathrm{SU}(2)$. They emphasized the analogy between this and the spin of the electron, which is also described by vectors in $\mathbb{C}^{2}$, acted on by the double cover of the $3 \mathrm{~d}$ rotation group, which is also $\mathrm{SU}(2)$. In keeping with this analogy, they invented a concept called "isospin". The proton was declared the isospin up state or $I_{3}=\frac{1}{2}$ state, and the neutron was declared the isospin down or $I_{3}=-\frac{1}{2}$ state. Cassen and Condon's paper put isospin on its way to becoming a useful tool in nuclear physics.

Isospin proved useful because it formalized the following idea, which emerged from empirical data around the time of Cassen and Condon's paper. Namely: the strong force, unlike the electromagnetic force, is the same whether the particles involved are protons or neutrons. Protons and neutrons are interchangeable, as long as we neglect the small difference in their mass, and most importantly, as long as we neglect electromagnetic effects. One can phrase this idea in terms of group representation theory as follows: the strong force is invariant under the action of $\mathrm{SU}(2)$.

Though this idea was later seen to be an oversimplification, it foreshadowed modern ideas about unification. The proton, living in the representation $\mathbb{C}$ of the trivial group, and the neutron, living in a different representation $\mathbb{C}$ of the trivial group, were unified into the nucleon, with representation $\mathbb{C}^{2}$ of $\mathrm{SU}(2)$. These symmetries hold for the strong force, but not for electromagnetism: we say this force "breaks" SU(2) symmetry.

But what does it mean, exactly, to say that a force is invariant under the action of some group? It means that when we are studying particles interacting via this force, the Hilbert space of each particle should be equipped with a unitary representation of this group. Moreover, any physical process caused by this force should be described by an "intertwining operator": that is, a linear operator that respects the action of this group. A bit more precisely, suppose $V$ and $W$ are finite-dimensional Hilbert spaces on which some group $G$ acts as unitary operators. Then a linear operator $F: V \rightarrow W$ is an intertwining operator if

$$
F(g \psi)=g F(\psi)
$$

for every $\psi \in V$ and $g \in G$.

Quite generally, symmetries give rise to conserved quantities. In quantum mechanics this works as follows. Suppose that $G$ is a Lie group with a unitary representation on the finite-dimensional Hilbert spaces $V$ and $W$. Then $V$ and $W$ automatically become representations of $\mathfrak{g}$, the Lie algebra of $G$, and any intertwining operator $F: V \rightarrow W$ respects the action of $\mathfrak{g}$. In other words,

$$
F(T \psi)=T F(\psi)
$$


for every $\psi \in V$ and $T \in \mathfrak{g}$. Next suppose that $\psi \in V$ is an eigenvector of $T$ :

$$
T \psi=i \lambda \psi
$$

for some real number $\lambda$. Then it is easy to check that $F(\psi)$ is again an eigenvector of $T$ with the same eigenvalue:

$$
T F(\psi)=i \lambda F(\psi) .
$$

So, the number $\lambda$ is "conserved" by the operator $F$.

The element $T \in \mathfrak{g}$ will act as a skew-adjoint operator on any unitary representation of $G$. Physicists prefer to work with selfadjoint operators since these have real eigenvalues. In quantum mechanics, selfadjoint operators are called "observables". We can get an observable by dividing $T$ by $i$.

In Casson and Condon's isospin theory of the strong interaction, the symmetry group $G$ is $\mathrm{SU}(2)$. The Lie algebra $\mathfrak{s u}(2)$ has a basis consisting of three elements, and the quantity $I_{3}$ arises as above: it is just the eigenvalue of one of these elements, divided by $i$ to get a real number. Because any physical process caused by the strong force is described by an intertwining operator, $I_{3}$ is conserved. In other words, the total $I_{3}$ of any system remains unchanged after a process that involves only strong interactions.

Nevertheless, for the states in $\mathbb{C}^{2}$ that mix protons and neutrons to have any meaning, there must be a mechanism which can convert protons into neutrons and vice versa. Mathematically, we have a way to do this: the action of $\mathrm{SU}(2)$. What does this correspond to, physically?

The answer originates in the work of Hideki Yukawa. In the early 1930s, he predicted the existence of a particle that mediates the strong force, much as the photon mediates the electromagnetic force. From known properties of the strong force, he was able to predict that this particle should be about 200 times as massive as the electron, or about a tenth the mass of a proton. He predicted that experimentalists would find a particle with a mass in this range, and that it would interact strongly when it collided with nuclei.

Partially because of the intervention of World War II, it took over ten years for Yukawa's prediction to be vindicated. After a famous false alarm (see Section 2.5), it became clear by 1947 that a particle with the expected properties had been found. It was called the pion and it came in three varieties: one with positive charge, the $\pi^{+}$, one neutral, the $\pi^{0}$, and one with negative charge, the $\pi^{-}$.

The pion proved to be the mechanism that can transform nucleons. To wit, we observe processes such as those in Figure 1, where we have drawn the Feynman diagrams which depict the nucleons absorbing pions, transforming where they are allowed to by charge conservation.

Because of isospin conservation, we can measure the $I_{3}$ of a pion by looking at these interactions with the nucleons. It turns out that the $I_{3}$ of a pion is the same as its charge:

\begin{tabular}{c|r}
\hline Pion & $I_{3}$ \\
\hline$\pi^{+}$ & +1 \\
$\pi^{0}$ & 0 \\
$\pi^{-}$ & -1 \\
\hline
\end{tabular}

Here we pause, because we can see the clearest example of a pattern that lies at the heart of the Standard Model. It is the relationship between isospin $I_{3}$ and charge 

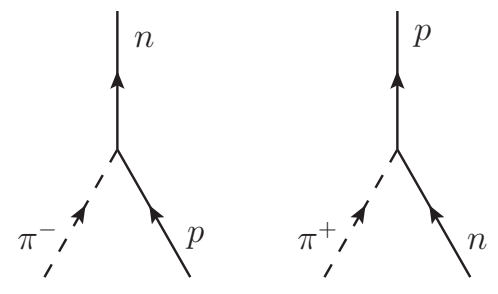

$$
\pi^{-}+p \rightarrow n
$$

$$
\pi^{+}+n \rightarrow p
$$
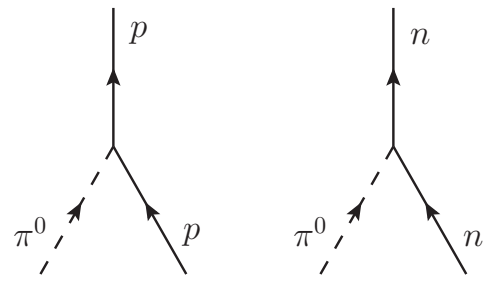

$$
\pi^{0}+p \rightarrow p \quad \pi^{0}+n \rightarrow n
$$

FigurE 1. The nucleons absorbing pions.

$Q$. For the pion, they are equal:

$$
Q(\pi)=I_{3}(\pi) .
$$

But they are also related for the nucleon, though in a subtler way:

\begin{tabular}{c|r|c}
\hline Nucleon & $I_{3}$ & Charge \\
\hline$p$ & $\frac{1}{2}$ & 1 \\
$n$ & $-\frac{1}{2}$ & 0 \\
\hline
\end{tabular}

The relationship for nucleons is

$$
Q(N)=I_{3}(N)+\frac{1}{2}
$$

This is nearly the most general relationship. It turns out that, for any given family of particles that differ only by $I_{3}$, we have the Gell-Mann-Nishijima formula:

$$
Q=I_{3}+Y / 2
$$

where $Q$ and $I_{3}$ depend on the particle, but a new quantity, the hypercharge $Y$, depends only on the family. For example, pions all have hypercharge $Y=0$, while nucleons both have hypercharge $Y=1$.

Mathematically, $Y$ being constant on "families" just means it is constant on representations of the isospin symmetry group, $\mathrm{SU}(2)$. The three pions, like the proton and neutron, are nearly identical in terms of mass and their strong interactions. In Heisenberg's theory, the different pions are just different isospin states of the same particle. Since there are three, they have to span a three-dimensional representation of $\mathrm{SU}(2)$. 
Up to isomorphism, there is only one three-dimensional complex irrep of $\mathrm{SU}(2)$, which is $\mathrm{Sym}^{2} \mathbb{C}^{2}$, the symmetric tensors of rank 2 . In general, the unique $(n+1)$ dimensional irrep of $\mathrm{SU}(2)$ is given by $\mathrm{Sym}^{n} \mathbb{C}^{2}$. Physicists call this the spin- $\boldsymbol{n} / \mathbf{2}$ representation of $\mathrm{SU}(2)$, or in the present context, the "isospin- $n / 2$ representation". This representation has a basis of vectors where $I_{3}$ ranges from $-n / 2$ to $n / 2$ in integer steps. Nucleons lie in the isospin- $\frac{1}{2}$ representation, while pions lie in the isospin-1 representation.

This sets up an interesting puzzle. We know two ways to transform nucleons: the mathematical action of SU(2), and their physical interactions with pions. How are these related?

The answer lies in the representation theory. Just as the two nucleons span the two-dimensional irrep of $\mathbb{C}^{2}$ of $\mathrm{SU}(2)$, the pions span the three-dimensional irrep $\mathrm{Sym}^{2} \mathbb{C}^{2}$ of $\mathrm{SU}(2)$. But there is another way to write this representation which sheds light on the pions and the way they interact with nucleons: because $\mathrm{SU}(2)$ is itself a three-dimensional real manifold, its Lie algebra $\mathfrak{s u}(2)$ is a three-dimensional real vector space. $\mathrm{SU}(2)$ acts on itself by conjugation, which fixes the identity and thus induces linear transformations of $\mathfrak{s u}(2)$, giving a representation of $\mathrm{SU}(2)$ on $\mathfrak{s u}(2)$ called the adjoint representation.

For simple Lie groups such as $\mathrm{SU}(2)$, the adjoint representation is irreducible. Thus $\mathfrak{s u}(2)$ is a three-dimensional real irrep of $\mathrm{SU}(2)$. This is different from the three-dimensional complex irrep $\mathrm{Sym}^{2} \mathbb{C}^{2}$, but very related. Indeed, $\mathrm{Sym}^{2} \mathbb{C}^{2}$ is just the complexification of $\mathfrak{s u}(2)$ :

$$
\operatorname{Sym}^{2} \mathbb{C}^{2} \cong \mathbb{C} \otimes \mathfrak{s u}(2) \cong \mathfrak{s l}(2, \mathbb{C})
$$

The pions thus live in $\mathfrak{s l}(2, \mathbb{C})$, a complex Lie algebra, and this acts on $\mathbb{C}^{2}$ because $\mathrm{SU}(2)$ does. To be precise, Lie group representations induce Lie algebra representations, so the real Lie algebra $\mathfrak{s u}(2)$ has a representation on $\mathbb{C}^{2}$. This then extends to a representation of the complex Lie algebra $\mathfrak{s l}(2, \mathbb{C})$. This representation is even familiar. It is the fundamental representation of $\mathfrak{s l}(2, \mathbb{C})$ on $\mathbb{C}^{2}$.

Quite generally, whenever $\mathfrak{g}$ is the Lie algebra of a Lie group $G$, and $\rho: G \times V \rightarrow$ $V$ is a representation of $G$ on some finite-dimensional vector space $V$, we get a representation of the Lie algebra $\mathfrak{g}$ on $V$, which we can think of as a linear map

$$
d \rho: \mathfrak{g} \otimes V \rightarrow V
$$

This map is actually an intertwining operator, meaning that it commutes with the action of $G$ : since $\mathfrak{g}$ and $V$ are both representations of $G$, this is a sensible thing to say, and it is easy to check.

Pions act on nucleons via precisely such an intertwining operator:

$$
\mathfrak{s l}(2, \mathbb{C}) \otimes \mathbb{C}^{2} \rightarrow \mathbb{C}^{2}
$$

So, the interaction between pions and nucleons arises naturally from the action of $\mathrm{SU}(2)$ on $\mathbb{C}^{2}$ after we complexify the Lie algebra of this group! 
Physicists have invented a nice way to depict such intertwining operators, namely, Feynman diagrams:

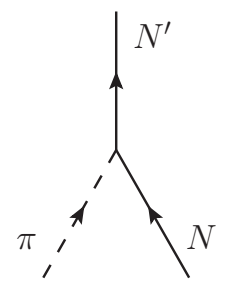

Figure 2. A nucleon absorbs a pion.

Here we see a nucleon coming in, absorbing a pion, and leaving. That is, this diagram depicts a basic interaction between pions and nucleons.

Feynman diagrams are calculational tools in physics, though to actually use them as such, we need quantum field theory. Then, instead of just standing for intertwining operators between representations of compact groups such as $\mathrm{SU}(2)$, they depict intertwining operators between representations of the product of this group and the Poincaré group, which describes the symmetries of spacetime. Unfortunately, the details are beyond the scope of this paper. By ignoring the Poincaré group, we are, in the language of physics, restricting our attention to "internal degrees of freedom", and their "internal" (i.e., gauge) symmetries.

Nonetheless, we can put basic interactions such as the one in Figure 2 together to form more complicated ones, such as this:

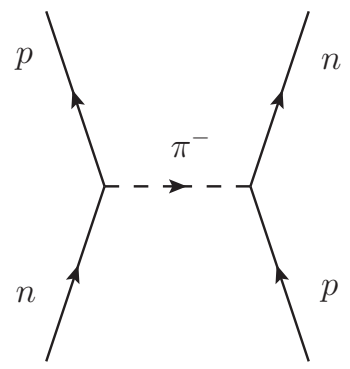

Here, two nucleons interact by exchanging pions. This is the mechanism for the strong force proposed by Yukawa, still considered approximately right today. Better, though, it depicts all the representation-theoretic ingredients of a modern gauge theory in physics. That is, it shows two nucleons, which live in a representation $\mathbb{C}^{2}$ of the gauge group $\mathrm{SU}(2)$, interacting by the exchange of a pion, which lives in the complexified adjoint representation $\mathbb{C} \otimes \mathfrak{s u}(2)$. In the coming sections we will see how these ideas underlie the Standard Model.

\subsection{The fundamental fermions.}

2.2.1. Quarks. In the last section, we learned how Heisenberg unified the proton and neutron into the nucleon, and that Yukawa proposed that nucleons interact by exchanging pions. This viewpoint turned out to be at least approximately true, but 
it was based on the idea that the proton, neutron and pions were all fundamental particles without internal structure, which was not ultimately supported by the evidence.

Protons and neutrons are not fundamental. They are made of particles called quarks. There are a number of different types of quarks, called flavors. However, it takes only two flavors to make protons and neutrons: the up quark, $u$, and the down quark, $d$. The proton consists of two up quarks and one down:

$$
p=u u d
$$

while the neutron consists of one up quark and two down:

$$
n=u d d
$$

Protons have an electric charge of +1 , exactly opposite the electron, while neutrons are neutral, with 0 charge. These two conditions are enough to determine the charge

\begin{tabular}{|c|c|c|}
\hline \multicolumn{3}{|c|}{ Fundamental Fermions (second try) } \\
\hline Name & Symbol & Charge \\
\hline Electron & $e^{-}$ & -1 \\
\hline Up quark & $u$ & $+\frac{2}{3}$ \\
\hline Down quark & $d$ & $-\frac{1}{3}$ \\
\hline
\end{tabular}
of their constituents, which are fundamental fermions much like the electron:

There are more quarks than these, but these are the lightest ones, comprising the first generation. They are all we need to make protons and neutrons, and so, with the electron in tow, the above list contains all the particles we need to make atoms.

Yet quarks, fundamental as they are, are never seen in isolation. They are always bunched up into particles such as the proton and neutron. This phenomenon is called confinement. It makes the long, convoluted history of how we came to understand quarks, despite the fact that they are never seen, all the more fascinating. Unfortunately, we do not have space for this history here, but it can be found in the books by Crease and Mann [8, Segrè [33, and Pais 25].

It is especially impressive how physicists were able to discover that each flavor of quark comes in three different states, called colors: red $r$, green $g$, and blue $b$. These "colors" have nothing to do with actual colors; they are just cute namesthough as we shall see, the names are quite well chosen. Mathematically, all that matters is that the Hilbert space for a single quark is $\mathbb{C}^{3}$; we call the standard basis vectors $r, g$ and $b$. The color symmetry group $\mathrm{SU}(3)$ acts on this Hilbert space in the obvious way, via its fundamental representation.

Since both up and down quarks come in three color states, there are really six kinds of quarks in the matter we see around us. Three up quarks, spanning a copy of $\mathbb{C}^{3}$ :

$$
u^{r}, u^{g}, u^{b} \in \mathbb{C}^{3} .
$$

and three down quarks, spanning another copy of $\mathbb{C}^{3}$ :

$$
d^{r}, d^{g}, d^{b} \in \mathbb{C}^{3} .
$$

The group SU(3) acts on each space. All six quarks taken together span this vector space:

$$
\mathbb{C}^{3} \oplus \mathbb{C}^{3} \cong \mathbb{C}^{2} \otimes \mathbb{C}^{3}
$$


where $\mathbb{C}^{2}$ is spanned by the flavors $u$ and $d$. Put another way, a first-generation quark comes in one of six flavor-color states.

How could physicists discover the concept of color, given that quarks are confined? In fact, confinement was the key to this discovery! Confinement amounts to the following decree: all observed states must be white, i.e., invariant under the action of $\mathrm{SU}(3)$. It turns out that this has many consequences.

For starters, this decree implies that we cannot see an individual quark, because they all transform nontrivially under $\mathrm{SU}(3)$. Nor do we ever see a particle built from two quarks, since no unit vectors in $\mathbb{C}^{3} \otimes \mathbb{C}^{3}$ are fixed by $\mathrm{SU}(3)$. But we do see particles made of three quarks: namely, nucleons! This is because there are unit vectors in

$$
\mathbb{C}^{3} \otimes \mathbb{C}^{3} \otimes \mathbb{C}^{3}
$$

fixed by $\mathrm{SU}(3)$. Indeed, as a representation of $\mathrm{SU}(3), \mathbb{C}^{3} \otimes \mathbb{C}^{3} \otimes \mathbb{C}^{3}$ contains precisely one copy of the trivial representation: the antisymmetric rank three tensors, $\Lambda^{3} \mathbb{C}^{3} \subseteq$ $\mathbb{C}^{3} \otimes \mathbb{C}^{3} \otimes \mathbb{C}^{3}$. This one-dimensional vector space is spanned by the wedge product of all three basis vectors:

$$
r \wedge b \wedge g \in \Lambda^{3} \mathbb{C}^{3} .
$$

So, up to normalization, this must be the color state of a nucleon. Now we see why the "color" terminology is well chosen: an equal mixture of red, green and blue light is white. This is just a coincidence, but it is too cute to resist.

So, color is deeply related to confinement. Flavor, on the other hand, is deeply related to isospin. Indeed, the flavor $\mathbb{C}^{2}$ is suspiciously like the isospin $\mathbb{C}^{2}$ of the nucleon. We even call the quark flavors "up" and "down". This is no accident. The proton and neutron, which are the two isospin states of the nucleon, differ only by their flavors, and only the flavor of one quark at that. If one could interchange $u$ and $d$, one could interchange protons and neutrons.

Indeed, we can use quarks to explain the isospin symmetry of Section 2.1. Protons and neutrons are so similar, with nearly the same mass and strong interactions, because $u$ and $d$ quarks are so similar, with nearly the same mass and truly identical colors.

So as in Section 2.1, let $\mathrm{SU}(2)$ act on the flavor states $\mathbb{C}^{2}$. By analogy with that section, we call this $\mathrm{SU}(2)$ the isospin symmetries of the quark model. Unlike the color symmetries $\mathrm{SU}(3)$, these symmetries are not exact, because $u$ and $d$ quarks have different mass and charge. Nevertheless, they are useful.

The isospin of the proton and neutron then arises from the isospin of its quarks. Define $I_{3}(u)=\frac{1}{2}$ and $I_{3}(d)=-\frac{1}{2}$, making $u$ and $d$ the isospin up and down states at which their names hint. To find the $I_{3}$ of a composite, such as a proton or neutron, add the $I_{3}$ for its constituents. This gives the proton and neutron the right $I_{3}$ :

$$
\begin{aligned}
& I_{3}(p)=\frac{1}{2}+\frac{1}{2}-\frac{1}{2}=\frac{1}{2} \\
& I_{3}(n)=\frac{1}{2}-\frac{1}{2}-\frac{1}{2}=-\frac{1}{2} .
\end{aligned}
$$

Of course, having the right $I_{3}$ is not the whole story for isospin. The states $p$ and $n$ must still span a copy of the fundamental representation $\mathbb{C}^{2}$ of $\mathrm{SU}(2)$. Whether or not this happens depends on how the constituent quark flavors transform under $\mathrm{SU}(2)$. 
The states $u \otimes u \otimes d$ and $u \otimes d \otimes d$ do not span a copy of the fundamental representation of $\mathrm{SU}(2)$ inside $\mathbb{C}^{2} \otimes \mathbb{C}^{2} \otimes \mathbb{C}^{2}$. So, as with color, the equations

$$
p=u u d, \quad n=u d d
$$

fail to give us the whole story. For the proton, we actually need some linear combination of the $I_{3}=\frac{1}{2}$ flavor states, which are made of two $u$ 's and one $d$ :

$$
u \otimes u \otimes d, \quad u \otimes d \otimes u, \quad d \otimes u \otimes u \quad \in \quad \mathbb{C}^{2} \otimes \mathbb{C}^{2} \otimes \mathbb{C}^{2} .
$$

For the neutron, we need some linear combination of the $I_{3}=-\frac{1}{2}$ flavor states, which are made of one $u$ and two d's:

$$
u \otimes d \otimes d, \quad d \otimes u \otimes d, \quad d \otimes d \otimes u \quad \in \quad \mathbb{C}^{2} \otimes \mathbb{C}^{2} \otimes \mathbb{C}^{2} .
$$

Writing $p=u u d$ and $n=u d d$ is just a sort of shorthand for saying that $p$ and $n$ are made from basis vectors with those quarks in them.

In physics, the linear combination required to make $p$ and $n$ work also involves the "spin" of the quarks, which lies outside of our scope. We will content ourselves with showing that it can be done. That is, we will show that $\mathbb{C}^{2} \otimes \mathbb{C}^{2} \otimes \mathbb{C}^{2}$ really does contain a copy of the fundamental representation $\mathbb{C}^{2}$ of $\mathrm{SU}(2)$. To do this, we use the fact that any rank 2 tensor can be decomposed into symmetric and antisymmetric parts; for example,

$$
\mathbb{C}^{2} \otimes \mathbb{C}^{2} \cong \operatorname{Sym}^{2} \mathbb{C}^{2} \oplus \Lambda^{2} \mathbb{C}^{2}
$$

and this is actually how $\mathbb{C}^{2} \otimes \mathbb{C}^{2}$ decomposes into irreps. $\mathrm{Sym}^{2} \mathbb{C}^{2}$, as we noted in Section 2.1, is the unique three-dimensional irrep of $\mathrm{SU}(2)$; its orthogonal complement $\Lambda^{2} \mathbb{C}^{2}$ in $\mathbb{C}^{2} \otimes \mathbb{C}^{2}$ is thus also a subrepresentation, but this space is one-dimensional, and must therefore be the trivial irrep, $\Lambda^{2} \mathbb{C}^{2} \cong \mathbb{C}$. In fact, for any $\mathrm{SU}(n)$, the top exterior power of its fundamental representation, $\Lambda^{n} \mathbb{C}^{n}$, is trivial.

As a representation of $\mathrm{SU}(2)$, we thus have

$$
\begin{aligned}
\mathbb{C}^{2} \otimes \mathbb{C}^{2} \otimes \mathbb{C}^{2} & \cong \mathbb{C}^{2} \otimes\left(\mathrm{Sym}^{2} \mathbb{C}^{2} \oplus \mathbb{C}\right) \\
& \cong \mathbb{C}^{2} \otimes \mathrm{Sym}^{2} \mathbb{C}^{2} \oplus \mathbb{C}^{2} .
\end{aligned}
$$

So indeed, $\mathbb{C}^{2}$ is a subrepresentation of $\mathbb{C}^{2} \otimes \mathbb{C}^{2} \otimes \mathbb{C}^{2}$.

As in the last section, there is no reason to have the full $\mathbb{C}^{2}$ of isospin states for nucleons unless there is a way to change protons into neutrons. There, we discussed how the pions provide this mechanism. The pions live in $\mathfrak{s l}(2, \mathbb{C})$, the complexification of the adjoint representation of $\mathrm{SU}(2)$, and this acts on $\mathbb{C}^{2}$ :

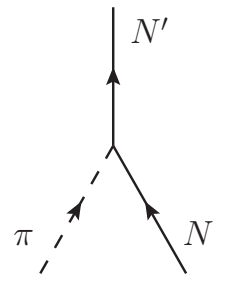

This Feynman diagram is a picture of the intertwining operator $\mathfrak{s l}(2, \mathbb{C}) \otimes \mathbb{C}^{2} \rightarrow$ $\mathbb{C}^{2}$ given by the representation of $\mathfrak{s l}(2, \mathbb{C})$ on $\mathbb{C}^{2}$. Now we know that nucleons are made of quarks and that isospin symmetry comes from their flavor symmetry. What about pions? 
Pions also fit into this model, but they require more explanation, because they are made of quarks and "antiquarks". To every kind of particle, there is a corresponding antiparticle, which is just like the original particle but with opposite charge and isospin. The antiparticle of a quark is called an antiquark.

In terms of group representations, passing from a particle to its antiparticle corresponds to taking the dual representation. Since the quarks live in $\mathbb{C}^{2} \otimes \mathbb{C}^{3}$, a representation of $\mathrm{SU}(2) \times \mathrm{SU}(3)$, the antiquarks live in the dual representation $\mathbb{C}^{2 *} \otimes \mathbb{C}^{3 *}$. Since $\mathbb{C}^{2}$ has basis vectors called up and down:

$$
u=\left(\begin{array}{l}
1 \\
0
\end{array}\right) \in \mathbb{C}^{2}, \quad d=\left(\begin{array}{l}
0 \\
1
\end{array}\right) \in \mathbb{C}^{2},
$$

the space $\mathbb{C}^{2 *}$ has a dual basis

$$
\bar{u}=(1,0) \in \mathbb{C}^{2 *}, \quad \bar{d}=(0,1) \in \mathbb{C}^{2 *},
$$

called antiup and antidown. Similarly, since the standard basis vectors for $\mathbb{C}^{3}$ are called red, green and blue, the dual basis vectors for $\mathbb{C}^{3 *}$ are known as anticolors: namely antired $\bar{r}$, antigreen $\bar{g}$, and antiblue $\bar{b}$. When it comes to actual colors of light, antired is called "cyan": this is the color of light which blended with red gives white. Similarly, antigreen is magenta, and antiblue is yellow. But few physicists dare speak of "magenta antiquarks"; apparently this would be taking the joke too far.

All pions are made from one quark and one antiquark. The flavor state of the pions must therefore live in

$$
\mathbb{C}^{2} \otimes \mathbb{C}^{2 *}
$$

We can use the fact that pions live in $\mathfrak{s l}(2, \mathbb{C})$ to find out how they decompose into quarks and antiquarks, since

$$
\mathfrak{s l}(2, \mathbb{C}) \subseteq \operatorname{End}\left(\mathbb{C}^{2}\right)
$$

First, express the pions as matrices:

$$
\pi^{+}=\left(\begin{array}{ll}
0 & 1 \\
0 & 0
\end{array}\right), \quad \pi^{0}=\left(\begin{array}{cc}
1 & 0 \\
0 & -1
\end{array}\right), \quad \pi^{-}=\left(\begin{array}{ll}
0 & 0 \\
1 & 0
\end{array}\right) .
$$

We know they have to be these matrices, up to normalization, because these act the right way on nucleons in $\mathbb{C}^{2}$ :

$$
\begin{gathered}
\pi^{-}+p \rightarrow n \\
\pi^{+}+n \rightarrow p \\
\pi^{0}+p \rightarrow p \\
\pi^{0}+n \rightarrow n .
\end{gathered}
$$

Now, apply the standard isomorphism $\operatorname{End}\left(\mathbb{C}^{2}\right) \cong \mathbb{C}^{2} \otimes \mathbb{C}^{2 *}$ to write these matrices as linear combinations of quarks and antiquarks:

$$
\pi^{+}=u \otimes \bar{d}, \quad \pi^{0}=u \otimes \bar{u}-d \otimes \bar{d}, \quad \pi^{-}=d \otimes \bar{u} .
$$

Note these all have the right $I_{3}$, because isospins reverse for antiparticles. For example, $I_{3}(\bar{d})=+\frac{1}{2}$, so $I_{3}\left(\pi^{+}\right)=1$.

In writing these pions as quarks and antiquarks, we have once again neglected to write the color, because this works the same way for all pions. As far as color goes, pions live in

$$
\mathbb{C}^{3} \otimes \mathbb{C}^{3 *}
$$


Confinement says that pions need to be white, just like nucleons, and there is only a one-dimensional subspace of $\mathbb{C}^{3} \otimes \mathbb{C}^{3 *}$ on which $\mathrm{SU}(3)$ acts trivially, spanned by

$$
r \otimes \bar{r}+g \otimes \bar{g}+b \otimes \bar{b} \in \mathbb{C}^{3} \otimes \mathbb{C}^{3 *}
$$

So, this must be the color state of all pions.

Finally, the Gell-Mann-Nishijima formula also still works for quarks, provided we define the hypercharge for both quarks to be $Y=\frac{1}{3}$ :

$$
\begin{aligned}
& Q(u)=I_{3}(u)+Y / 2=\frac{1}{2}+\frac{1}{6}=\frac{2}{3} \\
& Q(d)=I_{3}(d)+Y / 2=-\frac{1}{2}+\frac{1}{6}=-\frac{1}{3} .
\end{aligned}
$$

Since nucleons are made of three quarks, their total hypercharge is $Y=1$, just as before.

2.2.2. Leptons. With the quarks and electron, we have met all the fundamental fermions required to make atoms, and almost all of the particles we need to discuss

\begin{tabular}{|c|c|c|}
\hline \multicolumn{3}{|c|}{ The First Generation of Fermions-Charge } \\
\hline Name & Symbol & Charge \\
\hline Neutrino & $\nu$ & 0 \\
\hline Electron & $e^{-}$ & -1 \\
\hline Up quark & $u$ & $+\frac{2}{3}$ \\
\hline Down quark & $d$ & $-\frac{1}{3}$ \\
\hline
\end{tabular}
the Standard Model. Only one player remains to be introduced: the neutrino, $\nu$. This particle completes the first generation of fundamental fermions:

Neutrinos are particles which show up in certain interactions, such as the decay of a neutron into a proton, an electron, and an antineutrino

$$
n \rightarrow p+e^{-}+\bar{\nu} .
$$

Indeed, neutrinos $\nu$ have antiparticles $\bar{\nu}$, just like quarks and all other particles. The electron's antiparticle, denoted $e^{+}$, was the first discovered, so it wound up subject to an inconsistent naming convention: the "antielectron" is called a positron.

Neutrinos carry no charge and no color. They interact very weakly with other particles, so weakly that they were not observed until the 1950s, over 20 years after they were hypothesized by Pauli. Collectively, neutrinos and electrons, the fundamental fermions that do not feel the strong force, are called leptons.

In fact, the neutrino only interacts via the weak force. Like the electromagnetic force and the strong force, the weak force is a fundamental force, hypothesized to explain the decay of the neutron, and eventually required to explain other phenomena.

The weak force cares about the "handedness" of particles. It seems that every particle that we have discussed comes in left- and right-handed varieties, which (quite roughly speaking) spin in opposite ways. There are are left-handed leptons, which we denote as

$$
\nu_{L} \quad e_{L}^{-}
$$

and left-handed quarks, which we denote as

$$
u_{L} \quad d_{L}
$$


and similarly for right-handed fermions, which we will denote with a subscript $R$. As the terminology suggests, looking in a mirror interchanges left and right - in a mirror, the left-handed electron $e_{L}^{-}$looks like a right-handed electron, $e_{R}^{-}$, and vice versa. More precisely, applying any of the reflections in the Poincaré group to the (infinite-dimensional) representation we use to describe these fermions interchanges left and right.

Remarkably, the weak force interacts only with left-handed particles and righthanded antiparticles. For example, when the neutron decays, we always have

$$
n_{L} \rightarrow p_{L}+e_{L}^{-}+\bar{\nu}_{R}
$$

and never

$$
n_{R} \rightarrow p_{R}+e_{R}^{-}+\bar{\nu}_{L}
$$

This fact about the weak force, first noticed in the 1950s, left a deep impression on physicists. No other physical law is asymmetric in left and right. That is, no other physics, classical or quantum, looks different when viewed in a mirror. Why the weak force, and only the weak force, exhibits this behavior is a mystery.

Since neutrinos only feel the weak force, and the weak force only involves lefthanded particles, the right-handed neutrino $\nu_{R}$ has never been observed directly. For a long time, physicists believed this particle did not even exist, but recent observations of neutrino oscillations suggest otherwise. In this paper, we will assume there are right-handed neutrinos, but the reader should be aware that this is still open to some debate. In particular, even if they do exist, we know very little about them.

Note that isospin is not conserved in weak interactions. After all, we saw in the last section that $I_{3}$ is all about counting the number of $u$ quarks over the number of $d$ quarks. In a weak process such as neutron decay

$$
u d d \rightarrow u u d+e^{-}+\bar{\nu}
$$

the right-hand side has $I_{3}=-\frac{1}{2}$, while the left has $I_{3}=\frac{1}{2}$.

Yet maybe we are not being sophisticated enough. Perhaps isospin can be extended beyond quarks, and leptons can also carry $I_{3}$. Indeed, if we define $I_{3}\left(\nu_{L}\right)=\frac{1}{2}$ and $I_{3}\left(e^{-}\right)=-\frac{1}{2}$, we get

$$
\begin{aligned}
n_{L} & \rightarrow p_{L}+e_{L}^{-}+\bar{\nu}_{R} \\
I_{3}:-\frac{1}{2} & =\frac{1}{2}-\frac{1}{2}-\frac{1}{2}
\end{aligned}
$$

where we have used the rule that $I_{3}$ reverses sign for antiparticles.

This extension of isospin is called weak isospin since it extends the concept to weak interactions. Indeed, it turns out to be fundamental to the theory of weak interactions. Unlike regular isospin symmetry, which is only approximate, weak isospin symmetry turns out to be exact.

So from now on we shall discuss only weak isospin, and call it simply isospin. For this, $I_{3}$ equals zero for right-handed particles, and $\pm \frac{1}{2}$ for left-handed particles: 


\begin{tabular}{lccc}
\hline \multicolumn{3}{l}{ The First Generation of Fermions- } & Charge and Isospin \\
\hline Name & Symbol & $Q$ & $I_{3}$ \\
\hline Left-handed neutrino & $\nu_{L}$ & 0 & $\frac{1}{2}$ \\
Left-handed electron & $e_{L}^{-}$ & -1 & $-\frac{1}{2}$ \\
Left-handed up quark & $u_{L}$ & $+\frac{2}{3}$ & $\frac{1}{2}$ \\
Left-handed down quark & $d_{L}$ & $-\frac{1}{3}$ & $-\frac{1}{2}$ \\
Right-handed neutrino & $\nu_{R}$ & 0 & 0 \\
Right-handed electron & $e_{R}^{-}$ & -1 & 0 \\
Right-handed up quark & $u_{R}$ & $+\frac{2}{3}$ & 0 \\
Right-handed down quark & $d_{R}$ & $-\frac{1}{3}$ & 0 \\
\hline
\end{tabular}

The antiparticle of a left-handed particle is right-handed, and the antiparticle of a right-handed particle is left-handed. The isospins also change sign. For example, $I_{3}\left(e_{R}^{+}\right)=+\frac{1}{2}$, while $I_{3}\left(e_{L}^{+}\right)=0$.

In Section 2.3.2, we will see that the Gell-Mann-Nishijima formula, when applied to weak isospin, defines a fundamental quantity, the "weak hypercharge", that is vital to the Standard Model. But first, in Section 2.3.1, we discuss how to generalize the $\mathrm{SU}(2)$ symmetries from isospin to weak isospin.

\subsection{The fundamental forces.}

2.3.1. Isospin and $S U$ (2), Redux. The tale we told of isospin in Section 2.1 only concerned the strong force, which binds nucleons together into nuclei. We learned about an approximation in which nucleons live in the fundamental representation $\mathbb{C}^{2}$ of the isospin symmetry group $\mathrm{SU}(2)$, and that they interact by exchanging pions, which live in the complexified adjoint representation of this group, namely $\mathfrak{s l}(2, \mathbb{C})$.

But this tale is a mere prelude to the modern story, where weak isospin, defined in Section 2.2.2 is the star of the show. This story is not about the strong force, but rather the weak force. This story parallels the old one, but it involves lefthanded fermions instead of nucleons. The left-handed fermions, with $I_{3}= \pm \frac{1}{2}$, are paired up into fundamental representations of $\mathrm{SU}(2)$, the weak isospin symmetry group. There is one spanned by left-handed leptons:

$$
\nu_{L}, e_{L}^{-} \in \mathbb{C}^{2},
$$

and one spanned by each color of left-handed quarks:

$$
u_{L}^{r}, d_{L}^{r} \in \mathbb{C}^{2}, \quad u_{L}^{g}, d_{L}^{g} \in \mathbb{C}^{2}, \quad u_{L}^{b}, d_{L}^{b} \in \mathbb{C}^{2} .
$$

The antiparticles of the left-handed fermions, the right-handed antifermions, span the dual representation $\mathbb{C}^{2 *}$.

Because these particles are paired up in the same $\mathrm{SU}(2)$ representation, physicists often write them as doublets:

$$
\left(\begin{array}{c}
\nu_{L} \\
e_{L}^{-}
\end{array}\right) \quad\left(\begin{array}{c}
u_{L} \\
d_{L}
\end{array}\right)
$$

with the particle of higher $I_{3}$ written on top. Note that we have suppressed color on the quarks. This is conventional and is done because $\mathrm{SU}(2)$ acts the same way on all colors. 
The particles in these doublets then interact via the exchange of $W$ bosons, which are the weak isospin analogues of the pions. Like the pions, there are three $W$ bosons:

$$
W^{+}=\left(\begin{array}{ll}
0 & 1 \\
0 & 0
\end{array}\right), \quad W^{0}=\left(\begin{array}{cc}
1 & 0 \\
0 & -1
\end{array}\right), \quad W^{-}=\left(\begin{array}{ll}
0 & 0 \\
1 & 0
\end{array}\right) .
$$

They span the complexified adjoint representation of $\mathrm{SU}(2), \mathfrak{s l}(2, \mathbb{C})$, and they act on each of the doublets like the pions act on the nucleons, via the action of $\mathfrak{s l}(2, \mathbb{C})$ on $\mathbb{C}^{2}$. For example,

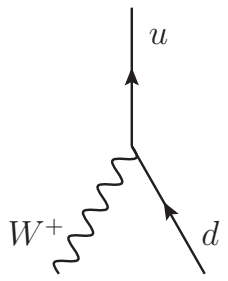

Again, Feynman diagrams are the physicists' way of drawing intertwining operators. Since all the $\mathbb{C}^{2}$ 's are acted on by the same $\mathrm{SU}(2)$, they can interact with each other via $W$ boson exchange. For example, quarks and leptons can interact via $W$ 's:

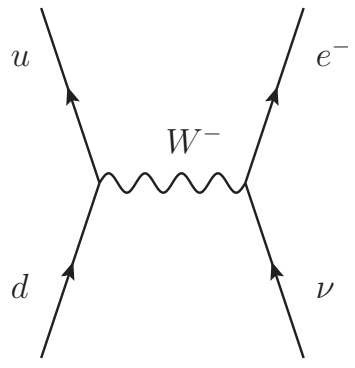

This is in sharp contrast to the old isospin theory. In the new theory, it is processes such as these that are responsible for the decay of the neutron:

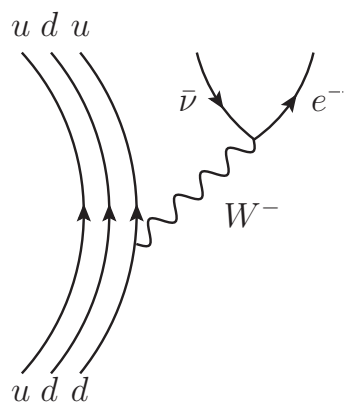

The fact that only left-handed particles are combined into doublets reflects the fact that only they take part in weak interactions. Every right-handed fermion, on the other hand, is trivial under $\mathrm{SU}(2)$. Each one spans the trivial representation C. An example is the right-handed electron

$$
e_{R}^{-} \in \mathbb{C}
$$


Physicists call these particles singlets, meaning they are trivial under $\mathrm{SU}(2)$. This is just the representation-theoretic way of saying that the right-handed electron, $e_{R}^{-}$, does not participate in weak interactions.

In summary, left-handed fermions are grouped into doublets (nontrivial representations of $\mathrm{SU}(2)$ on $\mathbb{C}^{2}$ ), while right-handed fermions are singlets (trivial representations on $\mathbb{C}$ ). So, the left-handed ones interact via the exchange of $W$ bosons, while the right-handed ones do not.

\begin{tabular}{|c|c|c|c|}
\hline \multicolumn{4}{|c|}{ The First Generation of Fermions-SU(2) Representations } \\
\hline Name & Symbol & $I_{3}$ & $\mathrm{SU}(2)$ representation \\
\hline Left-handed leptons & $\nu_{L}$ & $\pm \frac{1}{2}$ & $\mathbb{C}^{2}$ \\
\hline Left-handed quarks & $\begin{array}{l}u_{L} \\
d_{I}\end{array}$ & $\pm \frac{1}{2}$ & $\mathbb{C}^{2}$ \\
\hline Right-handed neutrino & $\nu_{R}$ & 0 & $\mathbb{C}$ \\
\hline Right-handed electron & $e_{R}^{-}$ & 0 & $\mathbb{C}$ \\
\hline Right-handed up quark & $u_{R}$ & 0 & $\mathbb{C}$ \\
\hline Right-handed down quark & $d_{R}$ & 0 & $\mathbb{C}$ \\
\hline
\end{tabular}

2.3.2. Hypercharge and $U(1)$. In Section 2.2.2 we saw how to extend the notion of isospin to weak isospin, which proved to be more fundamental, since we saw in Section 2.3.1 how this gives rise to interactions among left-handed fermions mediated via $W$ bosons.

We grouped all the fermions into $\mathrm{SU}(2)$ representations. When we did this in Section 2.1, we saw that the $\mathrm{SU}(2)$ representations of particles were labeled by a quantity, the hypercharge $Y$, which relates the isospin $I_{3}$ to the charge $Q$ via the Gell-Mann-Nishijima formula

$$
Q=I_{3}+Y / 2 \text {. }
$$

We can use this formula to extend the notion of hypercharge to weak hypercharge, a quantity which labels the weak isospin representations. For left-handed quarks, this notion, like weak isospin, coincides with the old isospin and hypercharge. We have weak hypercharge $Y=\frac{1}{3}$ for these particles:

$$
\begin{aligned}
& Q\left(u_{L}\right)=I_{3}\left(u_{L}\right)+Y / 2=\frac{1}{2}+\frac{1}{6}=\frac{2}{3} \\
& Q\left(d_{L}\right)=I_{3}\left(d_{L}\right)+Y / 2=-\frac{1}{2}+\frac{1}{6}=-\frac{1}{3} .
\end{aligned}
$$

But just as weak isospin extended isospin to leptons, weak hypercharge extends hypercharge to leptons. For left-handed leptons the Gell-Mann-Nishijima formula holds if we set $Y=-1$ :

$$
\begin{aligned}
& Q\left(\nu_{L}\right)=I_{3}\left(\nu_{L}\right)+Y / 2=\frac{1}{2}-\frac{1}{2}=0, \\
& Q\left(e_{L}^{-}\right)=I_{3}\left(e_{L}^{-}\right)+Y / 2=-\frac{1}{2}-\frac{1}{2}=-1 .
\end{aligned}
$$

Note that the weak hypercharge of quarks comes in units one-third the size of the weak hypercharge for leptons, a reflection of the fact that quark charges come in units one-third the size of lepton charges. Indeed, thanks to the Gell-MannNishijima formula, these facts are equivalent. 
For right-handed fermions, weak hypercharge is even simpler. Since $I_{3}=0$ for these particles, the Gell-Mann-Nishijima formula reduces to

$$
Q=Y / 2 .
$$

So, the hypercharge of a right-handed fermion is twice its charge. In summary, the fermions have these hypercharges:

\begin{tabular}{lcc}
\hline \multicolumn{2}{l}{ The First Generation of Fermions- } & Hypercharge \\
\hline Name & Symbol & $Y$ \\
\hline Left-handed leptons & $\left(\begin{array}{c}\nu_{L} \\
e_{L}^{-}\end{array}\right.$ & -1 \\
Left-handed quarks & $\left(\begin{array}{c}u_{L} \\
d_{L}\end{array}\right)$ & $\frac{1}{3}$ \\
Right-handed neutrino & $\nu_{R}$ & 0 \\
Right-handed electron & $e_{R}^{-}$ & -2 \\
Right-handed up quark & $u_{R}$ & $\frac{4}{3}$ \\
Right-handed down quark & $d_{R}$ & $-\frac{2}{3}$ \\
\hline
\end{tabular}

But what is the meaning of hypercharge? We can start by reviewing our answer for the quantity $I_{3}$. This quantity, as we have seen, is related to how particles interact via $W$ bosons, because particles with $I_{3}= \pm \frac{1}{2}$ span the fundamental representation of $\mathrm{SU}(2)$, while the $W$ bosons span the complexified adjoint representation, which acts on any other representation. Yet there is a deeper connection.

In quantum mechanics, observables such as $I_{3}$ correspond to selfadjoint operators. We will denote the operator corresponding to an observable with a caret; for example, $\hat{I}_{3}$ is the operator corresponding to $I_{3}$. A state of specific $I_{3}$, such as $\nu_{L}$, which has $I_{3}=\frac{1}{2}$, is an eigenvector,

$$
\hat{I}_{3} \nu_{L}=\frac{1}{2} \nu_{L}
$$

with an eigenvalue that is the $I_{3}$ of the state. This makes it easy to write $\hat{I}_{3}$ as a matrix when we let it act on the $\mathbb{C}^{2}$ with basis $\nu_{L}$ and $e_{L}^{-}$, or any other doublet. We get

$$
\hat{I}_{3}=\left(\begin{array}{cc}
\frac{1}{2} & 0 \\
0 & -\frac{1}{2}
\end{array}\right)
$$

Note that this is an element of $\mathfrak{s u}(2)$ divided by $i$. So, it lies in $\mathfrak{s l}(2, \mathbb{C})$, the complexified adjoint representation of $\mathrm{SU}(2)$. In fact it equals $\frac{1}{2} W^{0}$, one of the gauge bosons. So, up to a constant of proportionality, the observable $\hat{I}_{3}$ is one of the gauge bosons!

Similarly, corresponding to hypercharge $Y$ is an observable $\hat{Y}$. This is also, up to proportionality, a gauge boson, though this gauge boson lives in the complexified adjoint representation of $\mathrm{U}(1)$.

Here are the details. Particles with hypercharge $Y$ span irreps $\mathbb{C}_{Y}$ of $\mathrm{U}(1)$. Since $\mathrm{U}(1)$ is abelian, all of its irreps are one-dimensional. By $\mathbb{C}_{Y}$ we denote the one-dimensional vector space $\mathbb{C}$ with action of $\mathrm{U}(1)$ given by

$$
\alpha \cdot z=\alpha^{3 Y} z \text {. }
$$


The factor of 3 takes care of the fact that $Y$ might not be an integer, but is only guaranteed to be an integral multiple of $\frac{1}{3}$. For example, the left-handed leptons $\nu_{L}$ and $e_{L}^{-}$both have hypercharge $Y=-1$, so each one spans a copy of $\mathbb{C}_{-1}$ :

$$
\nu_{L} \in \mathbb{C}_{-1}, \quad e_{L}^{-} \in \mathbb{C}_{-1}
$$

or, more compactly,

$$
\nu_{L}, e_{L}^{-} \in \mathbb{C}_{-1} \otimes \mathbb{C}^{2}
$$

where $\mathbb{C}^{2}$ is trivial under $\mathrm{U}(1)$.

\begin{tabular}{|c|c|c|}
\hline The First Generation 0 & Fermior & U(1) Representati \\
\hline Name & Symbol & $\mathrm{U}(1)$ representation \\
\hline Left-handed leptons & $\nu_{L}$ & $\mathbb{C}_{-1}$ \\
\hline Left-handed quarks & $\left.\begin{array}{c}u_{L} \\
d_{L}\end{array}\right)$ & $\mathbb{C}_{\frac{1}{3}}$ \\
\hline Right-handed neutrino & $\nu_{R}$ & $\mathbb{C}_{0}$ \\
\hline Right-handed electron & $e_{R}^{-}$ & $\mathbb{C}_{-2}$ \\
\hline Right-handed up quark & $u_{R}$ & $\mathbb{C}_{\frac{4}{3}}$ \\
\hline Right-handed down quark & $d_{R}$ & $\mathbb{C}_{-\frac{2}{3}}$ \\
\hline
\end{tabular}

In summary, the fermions we have met thus far lie in these $\mathrm{U}(1)$ representations:

Now, the adjoint representation $\mathfrak{u}(1)$ of $U(1)$ is just the tangent space to the unit circle in $\mathbb{C}$ at 1 . It is thus parallel to the imaginary axis and can be identified as $i \mathbb{R}$. It is generated by $i$. $i$ also generates the complexification, $\mathbb{C} \otimes \mathfrak{u}(1) \cong \mathbb{C}$, though this also has other convenient generators, such as 1 . Given a particle $\psi \in \mathbb{C}_{Y}$ of hypercharge $Y$, we can differentiate the action of $\mathrm{U}(1)$ on $\psi$,

$$
e^{i \theta} \cdot \psi=e^{3 i Y \theta} \psi
$$

and set $\theta=0$ to find out how $\mathfrak{u}(1)$ acts:

$$
i \cdot \psi=3 i Y \psi
$$

Dividing by $i$ we obtain

$$
1 \cdot \psi=3 Y \psi
$$

In other words, we have

$$
\hat{Y}=\frac{1}{3} \in \mathbb{C}
$$

as an element of the complexified adjoint representation of $\mathrm{U}(1)$.

Particles with hypercharge interact by exchange of a boson, called the $B$ boson, which spans the complexified adjoint representation of $U(1)$. Of course, since $\mathbb{C}$ is one-dimensional, any nonzero element spans it. Up to a constant of proportionality, the $B$ boson is just $\hat{Y}$, and we might as well take it to be equal to $\hat{Y}$, but calling it $B$ is standard in physics.

The $B$ boson is a lot like another, more familiar $\mathrm{U}(1)$ gauge boson-the photon! The hypercharge force which the $B$ boson mediates is a lot like electromagnetism, which is mediated by photons, but its strength is proportional to hypercharge rather than charge. 
2.3.3. Electroweak symmetry breaking. In the Standard Model, electromagnetism and the weak force are unified into the electroweak force. This is a $\mathrm{U}(1) \times \mathrm{SU}(2)$ gauge theory, and without saying so, we just told you all about it in sections 2.3 .1 and 2.3.2. The fermions live in representations of hypercharge $\mathrm{U}(1)$ and weak isospin $\mathrm{SU}(2)$, exactly as we described in those sections, and we tensor these together to get representations of $\mathrm{U}(1) \times \mathrm{SU}(2)$ :

\begin{tabular}{|c|c|c|c|c|}
\hline \multicolumn{5}{|c|}{ The First Generation of Fermions $-\mathrm{U}(1) \times \mathrm{SU}(2)$ Representations } \\
\hline Name & Symbol & $Y$ & $I_{3}$ & $\mathrm{U}(1) \times \mathrm{SU}(2)$ representation \\
\hline Left-handed leptons & $\nu_{L}$ & -1 & $\pm \frac{1}{2}$ & $\mathbb{C}_{-1} \otimes \mathbb{C}^{2}$ \\
\hline Left-handed quarks & $\begin{array}{l}u_{L} \\
d_{L}\end{array}$ & $\frac{1}{3}$ & $\pm \frac{1}{2}$ & $\mathbb{C}_{\frac{1}{3}} \otimes \mathbb{C}^{2}$ \\
\hline Right-handed neutrino & $\nu_{R}$ & 0 & 0 & $\mathbb{C}_{0} \otimes \mathbb{C}$ \\
\hline Right-handed electron & $e_{R}^{-}$ & -2 & 0 & $\mathbb{C}_{-2} \otimes \mathbb{C}$ \\
\hline Right-handed up quark & $u_{R}$ & $\frac{4}{3}$ & 0 & $\mathbb{C}_{\frac{4}{3}} \otimes \mathbb{C}$ \\
\hline Right-handed down quark & $d_{R}$ & $-\frac{2}{3}$ & 0 & $\mathbb{C}_{-\frac{2}{3}} \otimes \mathbb{C}$ \\
\hline
\end{tabular}

These fermions interact by exchanging $B$ and $W$ bosons, which span $\mathbb{C} \oplus \mathfrak{s l}(2, \mathbb{C})$, the complexified adjoint representation of $\mathrm{U}(1) \times \mathrm{SU}(2)$.

Yet despite the electroweak unification, electromagnetism and the weak force are very different at low energies, including most interactions in the everyday world. Electromagnetism is a force of infinite range that we can describe by a $U(1)$ gauge theory, with the photon as gauge boson. The photon lives in $\mathbb{C} \oplus \mathfrak{s l}(2, \mathbb{C})$, alongside the $B$ and $W$ bosons. It is given by a linear combination

$$
\gamma=W^{0}+B / 2
$$

that parallels the Gell-Mann-Nishijima formula, $Q=I_{3}+Y / 2$. The weak force is of very short range and mediated by the $W$ and $Z$ bosons. The $Z$ boson lives in $\mathbb{C} \oplus \mathfrak{s l}(2, \mathbb{C})$ and is given by the linear combination

$$
Z=W^{0}-B / 2
$$

which is in some sense "perpendicular" to the photon. So, we can expand our chart of gauge bosons to include a basis for all of $\mathbb{C} \oplus \mathfrak{s l}(2, \mathbb{C})$ as follows:

\begin{tabular}{llc}
\hline \multicolumn{3}{c}{ Gauge Bosons (second try) } \\
\hline Force & Gauge boson & Symbol \\
\hline Electromagnetism & Photon & $\gamma$ \\
Weak force & $W$ and $Z$ bosons & $W^{+}, W^{-}$and $Z$ \\
\hline
\end{tabular}

What makes the photon (and electromagnetism) so different from the $W$ and $Z$ bosons (and the weak force)? It is symmetry breaking. Symmetry breaking allows the full electroweak $\mathrm{U}(1) \times \mathrm{SU}(2)$ symmetry group to be hidden away at high energy, replaced with the electromagnetic subgroup $U(1)$ at lower energies. This electromagnetic $U(1)$ is not the obvious factor of $U(1)$ given by $U(1) \times 1$. It is another copy, one which wraps around inside $\mathrm{U}(1) \times \mathrm{SU}(2)$ in a manner given by the Gell-Mann-Nishijima formula. 
The dynamics behind symmetry breaking are beyond the scope of this paper. We will just mention that, in the Standard Model, electroweak symmetry breaking is believed to be due to the "Higgs mechanism". In this mechanism, all particles in the Standard Model, including the photon and the $W$ and $Z$ bosons, interact with a particle called the "Higgs boson", and it is their differing interactions with this particle that makes them appear so different at low energies.

The Higgs boson has yet to be observed and remains one of the most mysterious parts of the Standard Model. As of this writing, the Large Hadron Collider at CERN is beginning operations; searching for the Higgs boson is one of its primary aims.

For the details on symmetry breaking and the Higgs mechanism, which is essential to understanding the Standard Model, see Huang [17. For a quick overview, see Zee [40].

2.3.4. Color and $S U(3)$. There is one more fundamental force in the Standard Model: the strong force. We have already met this force, as the force that keeps the nucleus together, but we discussed it before we knew that protons and neutrons are made of quarks. Now we need a force to keep quarks together inside the nucleons, and quark confinement tells us it must be a very strong force indeed. It is this force that, in modern parlance, is called the strong force and is considered fundamental. The force between nucleons is a side effect of these more fundamental interactions among quarks.

Like all three forces in the Standard Model, the strong force is explained by a gauge theory, this time with gauge group $\mathrm{SU}(3)$, the color symmetry group of the quarks. The picture is simpler than that of electromagnetism and the weak force, however, because this symmetry is "unbroken".

By now you can guess how this goes. Every kind of quark spans the fundamental representation $\mathbb{C}^{3}$ of $\mathrm{SU}(3)$. For example, the left-handed up quark, with its three colors, lives in

$$
u_{L}^{r}, u_{L}^{g}, u_{L}^{b} \in \mathbb{C}^{3}
$$

and the left-handed down quark, with its three colors, spans another copy of $\mathbb{C}^{3}$,

$$
d_{L}^{r}, d_{L}^{g}, d_{L}^{b} \in \mathbb{C}^{3}
$$

Together, these span the $\mathrm{SU}(3)$ representation

$$
\mathbb{C}^{2} \otimes \mathbb{C}^{3}
$$

where $\mathbb{C}^{2}$ is trivial under $\mathrm{SU}(3)$.

The quarks interact by the exchange of gluons, the gauge bosons of the strong force. These gauge bosons live in $\mathbb{C} \otimes \mathfrak{s u}(3) \cong \mathfrak{s l}(3, \mathbb{C})$, the complexified adjoint representation of $\mathrm{SU}(3)$. The interactions are drawn as Feynman diagrams, which now depict intertwining operators between representations of $\mathrm{SU}(3)$ :

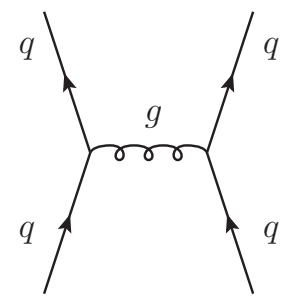


The gluons are fundamental particles, gauge bosons of the strong force, and they complete our table of gauge bosons:

\begin{tabular}{llc}
\hline & Gauge Bosons & \\
\hline Force & Gauge Boson & Symbol \\
\hline Electromagnetism & Photon & $\gamma$ \\
Weak force & $W$ and $Z$ bosons & $W^{+}, W^{-}$and $Z$ \\
Strong force & Gluons & $g$ \\
\hline
\end{tabular}

On the other hand, the leptons are "white": they transform trivially under $\mathrm{SU}(3)$. So, they do not exchange gluons. In other words, they are not affected by the strong force. We can capture all of this information in a table, where we give the $\mathrm{SU}(3)$ representations in which all our fermions live.

\begin{tabular}{|c|c|c|c|}
\hline \multicolumn{4}{|c|}{ The First Generation of Fermions-SU(3) Representations } \\
\hline Name & Symbol & Colors & $\mathrm{SU}(3)$ representation \\
\hline Left-handed neutrino & $\nu_{L}$ & white & $\mathbb{C}$ \\
\hline Left-handed electron & $e_{L}^{-}$ & white & $\mathbb{C}$ \\
\hline Left-handed up quarks & $u_{L}^{r}, u_{L}^{g}, u_{L}^{b}$ & $r, g, b$ & $\mathbb{C}^{3}$ \\
\hline Left-handed down quarks & $d_{L}^{r}, d_{L}^{g}, d_{L}^{b}$ & $r, g, b$ & $\mathbb{C}^{3}$ \\
\hline Right-handed electron & $e_{R}^{-}$ & white & $\mathbb{C}$ \\
\hline Right-handed neutrino & $\nu_{R}$ & white & $\mathbb{C}$ \\
\hline Right-handed up quarks & $u_{R}^{r}, u_{R}^{g}, u_{R}^{b}$ & $r, g, b$ & $\mathbb{C}^{3}$ \\
\hline Right-handed down quarks & $d_{R}^{r}, d_{R}^{g}, d_{R}^{b}$ & $r, g, b$ & $\mathbb{C}^{3}$ \\
\hline
\end{tabular}

2.4. The Standard Model representation. We are now in a position to put the entire Standard Model together in a single picture, much as we combined the isospin $\mathrm{SU}(2)$ and hypercharge $\mathrm{U}(1)$ into the electroweak gauge group, $\mathrm{U}(1) \times \mathrm{SU}(2)$, in Section 2.3.3. We then tensored the hypercharge $\mathrm{U}(1)$ representations with the isospin $\mathrm{SU}(2)$ representations to get the electroweak representations.

Now let us take this process one step further, by bringing in a factor of $\mathrm{SU}(3)$, for the color symmetry, and tensoring the representations of $\mathrm{U}(1) \times \mathrm{SU}(2)$ with the representations of SU(3). Doing this, we get the Standard Model. The Standard Model has this gauge group:

$$
G_{\mathrm{SM}}=\mathrm{U}(1) \times \mathrm{SU}(2) \times \mathrm{SU}(3) .
$$

The fundamental fermions described by the Standard Model combine to form representations of this group. We know what these are, and we describe all of them in Table 1 . 
TABLE 1. Fundamental fermions as representations of $G_{\mathrm{SM}}=$ $\mathrm{U}(1) \times \mathrm{SU}(2) \times \mathrm{SU}(3)$

\begin{tabular}{lcc}
\hline \multicolumn{3}{c}{ The Standard Model Representation } \\
\hline Name & Symbol & $\mathrm{U}(1) \times \mathrm{SU}(2) \times \mathrm{SU}(3)$ representation \\
\hline Left-handed leptons & $\left(\begin{array}{c}\nu_{L} \\
e_{L}^{-}\end{array}\right)$ & $\mathbb{C}_{-1} \otimes \mathbb{C}^{2} \otimes \mathbb{C}$ \\
Left-handed quarks & $\left(\begin{array}{c}u_{L}^{r}, u_{L}^{g}, u_{L}^{b} \\
d_{L}^{r}, d_{L}^{g}, d_{L}^{b}\end{array}\right)$ & $\mathbb{C}_{\frac{1}{3}} \otimes \mathbb{C}^{2} \otimes \mathbb{C}^{3}$ \\
Right-handed neutrino & $\nu_{R}$ & $\mathbb{C}_{0} \otimes \mathbb{C} \otimes \mathbb{C}$ \\
Right-handed electron & $e_{R}^{-}$ & $\mathbb{C}_{-2} \otimes \mathbb{C} \otimes \mathbb{C}$ \\
Right-handed up quarks & $\left(u_{R}^{r}, u_{R}^{g}, u_{R}^{b}\right)$ & $\mathbb{C}_{\frac{4}{3}} \otimes \mathbb{C} \otimes \mathbb{C}^{3}$ \\
Right-handed down quarks & $\left(d_{R}^{r}, d_{R}^{g}, d_{R}^{b}\right)$ & $\mathbb{C}_{-\frac{2}{3}} \otimes \mathbb{C} \otimes \mathbb{C}^{3}$ \\
\hline
\end{tabular}

All of the representations of $G_{\mathrm{SM}}$ in the left-hand column are irreducible, since they are made by tensoring irreps of this group's three factors, $\mathrm{U}(1), \mathrm{SU}(2)$ and $\mathrm{SU}(3)$. This is a general fact: if $V$ is an irrep of $G$, and $W$ is an irrep of $H$, then $V \otimes W$ is an irrep of $G \times H$. Moreover, all irreps of $G \times H$ arise in this way.

On the other hand, if we take the direct sum of all these irreps,

$$
F=\left(\mathbb{C}_{-1} \otimes \mathbb{C}^{2} \otimes \mathbb{C}\right) \quad \oplus \quad \cdots \quad \oplus \quad\left(\mathbb{C}_{-\frac{2}{3}} \otimes \mathbb{C} \otimes \mathbb{C}^{3}\right),
$$

we get a reducible representation containing all the first-generation fermions in the Standard Model. We call $F$ the fermion representation. If we take the dual of $F$, we get a representation describing all the antifermions in the first generation, and taking the direct sum of these spaces

$$
F \oplus F^{*},
$$

we get a representation of $G_{\mathrm{SM}}$ that we will call the Standard Model representation. It contains all the first-generation elementary particles in the Standard Model. It does not contain the gauge bosons or the mysterious Higgs.

The fermions living in the Standard Model representation interact by exchanging gauge bosons that live in the complexified adjoint representation of $G_{\mathrm{SM}}$. We have already met all of these, and we collect them in Table 2 .

TABLE 2. Gauge bosons

\begin{tabular}{llc}
\hline \multicolumn{3}{l}{ Gauge Bosons } \\
\hline Force & Gauge Boson & Symbol \\
\hline Electromagnetism & Photon & $\gamma$ \\
Weak force & $W$ and $Z$ bosons & $W^{+}, W^{-}$and $Z$ \\
Strong force & Gluons & $g$ \\
\hline
\end{tabular}

Of all the particles and antiparticles in $F \oplus F^{*}$, exactly two of them are fixed by the action of $G_{\mathrm{SM}}$. These are the right-handed neutrino

$$
\nu_{R} \in \mathbb{C}_{0} \otimes \mathbb{C} \otimes \mathbb{C}
$$


and its antiparticle

$$
\bar{\nu}_{L} \in\left(\mathbb{C}_{0} \otimes \mathbb{C} \otimes \mathbb{C}\right)^{*},
$$

both of which are trivial representations of $G_{\mathrm{SM}}$; they thus do not participate in any forces mediated by the gauge bosons of the Standard Model. They might interact with the Higgs boson, but very little about right-handed neutrinos is known with certainty at this time.

2.5. Generations. Our description of the Standard Model is almost at an end. We have told you about its gauge group, $G_{\mathrm{SM}}$, its representation $F \oplus F^{*}$ on the firstgeneration of fermions and antifermions, and a bit about how these fermions interact by exchanging gauge bosons, which live in the complexified adjoint representation of $G_{\mathrm{SM}}$. For the grand unified theories we are about to discuss, that is all we need. The stage is set.

Yet we would be derelict in our duty if we did not mention the second and third generation of fermions. The first evidence for these came in the 1930s, when a charged particle 207 times as heavy as the electron was found. At first researchers thought it was the particle predicted by Yukawa - the one that mediates the strong force between nucleons. But then it turned out the newly discovered particle was not affected by the strong force. This came as a complete surprise. As the physicist Rabi quipped at the time: "Who ordered that?"

Dubbed the muon and denoted $\mu^{-}$, this new particle turned out to act like an overweight electron. Like the electron, it feels only the electromagnetic and weak force - and like the electron, it has its own neutrino! So, the neutrino we have been discussing so far is now called the electron neutrino, $\nu_{e}$, to distinguish it from the muon neutrino, $\nu_{\mu}$. Together, the muon and the muon neutrino comprise the second generation of leptons. The muon decays via the weak force into an electron, a muon neutrino, and an electron antineutrino:

$$
\mu^{-} \rightarrow e^{-}+\nu_{\mu}+\bar{\nu}_{e}
$$

Much later, in the 1970s, physicists realized there was also a second generation of quarks: the charm quark, $c$, and the strange quark, $s$. This was evidence of another pattern in the Standard Model: there are as many flavors of quark as there are leptons. In Section 3.3, we will learn about the Pati-Salam model, which explains this pattern by unifying quarks and leptons.

\begin{tabular}{|c|c|c|c|c|c|}
\hline \multicolumn{6}{|c|}{ Quarks by Generation } \\
\hline \multicolumn{2}{|c|}{ 1st Generation } & \multicolumn{2}{|c|}{ 2nd Generation } & \multicolumn{2}{|c|}{ 3rd Generation } \\
\hline Name & Symbol & Name & Symbol & Name & Symbol \\
\hline $\mathrm{Up}$ & $u$ & Charm & $c$ & Top & $t$ \\
\hline Down & $d$ & Strange & $s$ & Bottom & $b$ \\
\hline
\end{tabular}

Today, we know about three generations of fermions. Three of quarks:

and three of leptons: 


\begin{tabular}{lc|cc|lc}
\hline \multicolumn{5}{c}{ Leptons by Generation } \\
\hline \multicolumn{1}{c}{ 1st Generation } & \multicolumn{2}{c}{ 2nd Generation } & \multicolumn{2}{c}{ 3rd Generation } \\
\hline Name & Symbol & Name & Symbol & Name & Symbol \\
\hline Electron & $\nu_{e}$ & Muon & $\nu_{\mu}$ & Tau & $\nu_{\tau}$ \\
neutrino & & neutrino & & neutrino & \\
Electron & $e^{-}$ & Muon & $\mu^{-}$ & Tau & $\tau^{-}$ \\
\hline
\end{tabular}

The second and third generations of quarks and charged leptons differ from the first by being more massive and able to decay into particles of the earlier generations. The various neutrinos do not decay, and for a long time it was thought they were massless, but now it is known that some and perhaps all of them are massive. This allows them to change back and forth from one type to another, a phenomenon called "neutrino oscillation".

The Standard Model explains all of this by something called the Higgs mechanism. Apart from how they interact with the Higgs boson, the generations are identical. For instance, as representations of $G_{\mathrm{SM}}$, each generation spans another copy of $F$. Each generation of fermions has corresponding antifermions, spanning a copy of $F^{*}$.

All told, we thus have three copies of the Standard Model representation, $F \oplus F^{*}$. We will only need to discuss one generation, so we find it convenient to speak as if $F \oplus F^{*}$ contains particles of the first generation. No one knows why the Standard Model is this redundant, with three sets of very similar particles. It remains a mystery.

\section{GRAND UNIFIED THEORIES}

Not all of the symmetries of $G_{\mathrm{SM}}$, the gauge group of the Standard Model, are actually seen in ordinary life. This is because some of the symmetries are "spontaneously broken". This means that while they are symmetries of the laws of physics, they are not symmetries of the vacuum. To see these symmetries we need to do experiments at very high energies, where the asymmetry of the vacuum has less effect. So, the behavior of particles at lower energies is like a shadow of the fundamental laws of physics, cast down from on high: a cryptic clue we must struggle to interpret.

It is reasonable to ask if this process continues. Could the symmetries of the Standard Model be just a subset of all the symmetries in nature? Could they be the low energy shadows of laws still more symmetric?

A grand unified theory, or GUT, constitutes a guess at what these "more symmetric" laws might be. It is a theory with more symmetry than the Standard Model, which reduces to the Standard Model at lower energies. It is also, therefore, an attempt to describe the physics at higher energies.

GUTs are speculative physics. The Standard Model has been tested in countless experiments. There is a lot of evidence that it is an incomplete theory, and some vague clues about what the next theory might be like, but so far there is no empirical evidence that any GUT is correct - and even some empirical evidence that some GUTs, like SU(5), are incorrect.

Nonetheless, GUTs are interesting to theoretical physicists, because they allow us to explore some very definite ideas about how to extend the Standard Model. Since they are based almost entirely on the representation theory of compact Lie groups, 
the underlying physical ideas provide a marvelous playground for this beautiful area of mathematics.

Amazingly, this beauty then becomes a part of the physics. The representation of $G_{\mathrm{SM}}$ used in the Standard Model seems ad hoc. Why this one? Why are all those seemingly arbitrary hypercharges floating around, mucking up some otherwise simple representations? Why do both leptons and quarks come in left- and righthanded varieties, which transform so differently? Why do quarks come in charges which are in units $\frac{1}{3}$ times an electron's charge? Why are there the same number of quarks and leptons? GUTs can shed light on these questions, using only group representation theory.

3.1. The SU(5) GUT. The SU(5) grand unified theory appeared in a 1974 paper by Howard Georgi and Sheldon Glashow 12. It was the first grand unified theory and is still considered the prototypical example. As such, there are many accounts of it in the physics literature. The textbooks by Ross 31 and Mohapatra 21] both devote an entire chapter to the $\mathrm{SU}(5)$ theory, and a lucid summary can be found in a review article by Witten [39], which also discusses the supersymmetric generalization of this theory.

In this section, we will limit our attention to the nonsupersymmetric version of $\mathrm{SU}(5)$ theory, which is how it was originally proposed. Unfortunately, this theory has since been ruled out by experiment; it predicts that protons will decay faster than the current lower bound on proton lifetime [26]. Nevertheless, because of its prototypical status and intrinsic interest, we simply must talk about the $\mathrm{SU}(5)$ theory.

The core idea behind the SU(5) grand unified theory is that because the Standard Model representation $F \oplus F^{*}$ is 32-dimensional, each particle or antiparticle in the first generation of fermions can be named by a 5 -bit code. Roughly speaking, these bits are the answers to five yes-or-no questions:

- Is the particle isospin up?

- Is it isospin down?

- Is it red?

- Is it green?

- Is it blue?

There are subtleties involved when we answer "yes" to both of the first two questions, or "yes" to more than one of the last three, but let us start with an example where these issues do not arise: the bit string 01100. This names a particle that is down and red. So, it refers to a red quark whose isospin is down, meaning $-\frac{1}{2}$. Glancing at Table 1, we see just one particle meeting this description: the red left-handed down quark, $d_{L}^{r}$.

We can flesh out this scheme by demanding that the operation of taking antiparticles correspond to switching 0's for 1's in the code. So the code for the antiparticle of $d_{L}^{r}$, the "antired right-handed antidown antiquark", is 10011. This is cute: it means that being antidown is the same as being up, while being antired is the same as being both green and blue.

Furthermore, in this scheme all antileptons are "black" (the particles with no color, ending in 000), while leptons are "white" (the particles with every color, ending in 111). Quarks have exactly one color, and antiquarks have exactly two. 
We are slowly working our way to the SU(5) theory. Next let us bring Hilbert spaces into the game. We can take the basic properties of being up, down, red, green or blue, and treat them as basis vectors for $\mathbb{C}^{5}$. Let us call these vectors $u, d, r, g, b$. The exterior algebra $\Lambda \mathbb{C}^{5}$ has a basis given by wedge products of these 5 vectors. This exterior algebra is 32 -dimensional, and it has a basis labelled by 5-bit strings. For example, the bit string 01100 corresponds to the basis vector $d \wedge r$, while the bit string 10011 corresponds to $u \wedge g \wedge b$.

Next we bring in representation theory. The group SU(5) has an obvious representation on $\mathbb{C}^{5}$. Since the operation of taking exterior algebras is functorial, this group also has a representation on $\Lambda \mathbb{C}^{5}$. In the $\mathrm{SU}(5)$ grand unified theory, this is the representation we use to describe a single generation of fermions and their antiparticles.

Just by our wording, though, we are picking out a splitting of $\mathbb{C}^{5}$ into $\mathbb{C}^{2} \oplus \mathbb{C}^{3}$ : the isospin and color parts, respectively. Choosing such a splitting of $\mathbb{C}^{5}$ picks out a subgroup of SU(5), the set of all group elements that preserve this splitting. This subgroup consists of block diagonal matrices with a $2 \times 2$ block and a $3 \times 3$ block, both unitary, such that the determinant of the whole matrix is 1 . Let us denote this subgroup as $\mathrm{S}(\mathrm{U}(2) \times \mathrm{U}(3))$.

Now for the miracle: the subgroup $\mathrm{S}(\mathrm{U}(2) \times \mathrm{U}(3))$ is isomorphic to the Standard Model gauge group (at least modulo a finite subgroup), and, when we restrict the representation of $\mathrm{SU}(5)$ on $\Lambda \mathbb{C}^{5}$ to $\mathrm{S}(\mathrm{U}(2) \times \mathrm{U}(3))$, we get the Standard Model representation!

There are two great things about this. The first is that it gives a concise and mathematically elegant description of the Standard Model representation. The second is that the seemingly ad hoc hypercharges in the Standard Model must be exactly what they are for this description to work. So, physicists say the SU(5) theory explains the fractional charges of quarks: the fact that quark charges come in units $\frac{1}{3}$ the size of the electron charge pops right out of this theory.

With this foretaste of the fruits the SU(5) theory will bear, let us get to work and sow the seeds. Our work will have two parts. First we need to check that

$$
\mathrm{S}(\mathrm{U}(2) \times \mathrm{U}(3)) \cong G_{\mathrm{SM}} / N
$$

where $N$ is some finite normal subgroup that acts trivially on $F \oplus F^{*}$. Then we need to check that indeed

$$
\Lambda \mathbb{C}^{5} \cong F \oplus F^{*}
$$

as representations of $\mathrm{S}(\mathrm{U}(2) \times \mathrm{U}(3))$.

First, we check the group isomorphism. Naively, one might seek to build the $\mathrm{SU}(5)$ theory by including $G_{\mathrm{SM}}$ as a subgroup of SU(5). Can this be done? Clearly, we can include $\mathrm{SU}(2) \times \mathrm{SU}(3)$ as block diagonal matrices in $\mathrm{SU}(5)$ :

$$
\begin{array}{clc}
\mathrm{SU}(2) \times \mathrm{SU}(3) & \rightarrow & \mathrm{SU}(5) \\
(g, h) & \longmapsto & \left(\begin{array}{cc}
g & 0 \\
0 & h
\end{array}\right),
\end{array}
$$

but this is not enough, because $G_{\mathrm{SM}}$ also has that pesky factor of $\mathrm{U}(1)$, related to the hypercharge. How can we fit that in?

The first clue is that elements of $\mathrm{U}(1)$ must commute with the elements of $\mathrm{SU}(2) \times$ $\mathrm{SU}(3)$. But the only elements of $\mathrm{SU}(5)$ that commute with everybody in the $\mathrm{SU}(2) \times$ $\mathrm{SU}(3)$ subgroup are diagonal, since they must separately commute with $\mathrm{SU}(2) \times 1$ 
and $1 \times \mathrm{SU}(3)$, and the only elements doing so are diagonal. Moreover, they must be scalars on each block. So, they have to look like this:

$$
\left(\begin{array}{ll}
\alpha & 0 \\
0 & \beta
\end{array}\right),
$$

where $\alpha$ stands for the $2 \times 2$ identity matrix times the complex number $\alpha \in \mathrm{U}(1)$, and similarly for $\beta$ in the $3 \times 3$ block. For the above matrix to lie in $\mathrm{SU}(5)$, it must have determinant 1 , so $\alpha^{2} \beta^{3}=1$. This condition cuts the group of such matrices from $\mathrm{U}(1) \times \mathrm{U}(1)$ down to $\mathrm{U}(1)$. In fact, all such matrices are of the form

$$
\left(\begin{array}{cc}
\alpha^{3} & 0 \\
0 & \alpha^{-2}
\end{array}\right)
$$

where $\alpha$ runs over $\mathrm{U}(1)$.

So if we throw in elements of this form, do we get $\mathrm{U}(1) \times \mathrm{SU}(2) \times \mathrm{SU}(3)$ ? More precisely, does this map:

$$
\begin{aligned}
\phi: \quad G_{\mathrm{SM}} & \rightarrow \\
(\alpha, g, h) & \longmapsto\left(\begin{array}{cc}
\alpha^{3} g & 0 \\
0 & \alpha^{-2} h
\end{array}\right)
\end{aligned}
$$

give an isomorphism between $G_{\mathrm{SM}}$ and $\mathrm{S}(\mathrm{U}(2) \times \mathrm{U}(3))$ ? It is clearly a homomorphism. It clearly maps $G_{\mathrm{SM}}$ into the subgroup $\mathrm{S}(\mathrm{U}(2) \times \mathrm{U}(3))$, and it is easy to check that it maps $G_{\mathrm{SM}}$ onto this subgroup. But is it one-to-one?

The answer is no: the map $\phi$ has a kernel, $\mathbb{Z}_{6}$. The kernel is the set of all elements of the form

$$
\left(\alpha, \alpha^{-3}, \alpha^{2}\right) \in \mathrm{U}(1) \times \mathrm{SU}(2) \times \mathrm{SU}(3)
$$

and this is $\mathbb{Z}_{6}$, because scalar matrices $\alpha^{-3}$ and $\alpha^{2}$ live in $\mathrm{SU}(2)$ and $\mathrm{SU}(3)$, respectively, if and only if $\alpha$ is a sixth root of unity. So, all we get is

$$
G_{\mathrm{SM}} / \mathbb{Z}_{6} \cong \mathrm{S}(\mathrm{U}(2) \times \mathrm{U}(3)) \hookrightarrow \mathrm{SU}(5) .
$$

This sets up a nerve-wracking test that the SU(5) theory must pass for it to have any chance of success. After all, not all representations of $G_{\mathrm{SM}}$ factor through $G_{\mathrm{SM}} / \mathbb{Z}_{6}$, but all those coming from representations of SU(5) must do so. A representation of $G_{\mathrm{SM}}$ will factor through $G_{\mathrm{SM}} / \mathbb{Z}_{6}$ only if the $\mathbb{Z}_{6}$ subgroup acts trivially.

In short: the $\mathrm{SU}(5)$ GUT is doomed unless $\mathbb{Z}_{6}$ acts trivially on every fermion (and antifermion, but that amounts to the same thing). For this to be true, some nontrivial relations between hypercharge, isospin and color must hold.

For example, consider the left-handed electron

$$
e_{L}^{-} \in \mathbb{C}_{-1} \otimes \mathbb{C}^{2} \otimes \mathbb{C} \text {. }
$$

For any sixth root of unity $\alpha$, we need

$$
\left(\alpha, \alpha^{-3}, \alpha^{2}\right) \in \mathrm{U}(1) \times \mathrm{SU}(2) \times \mathrm{SU}(3)
$$

to act trivially on this particle. Let us see how it acts. Note that:

- $\alpha \in \mathrm{U}(1)$ acts on $\mathbb{C}_{-1}$ as multiplication by $\alpha^{-3}$;

- $\alpha^{-3} \in \mathrm{SU}(2)$ acts on $\mathbb{C}^{2}$ as multiplication by $\alpha^{-3}$;

- $\alpha^{2} \in \mathrm{SU}(3)$ acts trivially on $\mathbb{C}$. 
So, we have

$$
\left(\alpha, \alpha^{-3}, \alpha^{2}\right) \cdot e_{L}^{-}=\alpha^{-3} \alpha^{-3} e_{L}^{-}=e_{L}^{-} .
$$

The action is indeed trivial, precisely because $\alpha$ is a sixth root of unity.

Or, consider the right-handed $d$ quark:

$$
d_{R} \in \mathbb{C}_{-\frac{2}{3}} \otimes \mathbb{C} \otimes \mathbb{C}^{3} .
$$

How does $\left(\alpha, \alpha^{-3}, \alpha^{2}\right)$ act on this? We note:

- $\alpha \in \mathrm{U}(1)$ acts on $\mathbb{C}_{-\frac{2}{3}}$ as multiplication by $\alpha^{-2}$;

- $\alpha^{-3} \in \mathrm{SU}(2)$ acts trivially on the trivial representation $\mathbb{C}$;

- $\alpha^{2} \in \mathrm{SU}(3)$ acts on $\mathbb{C}^{3}$ as multiplication by $\alpha^{2}$.

So, we have

$$
\left(\alpha, \alpha^{-3}, \alpha^{2}\right) \cdot d_{R}=\alpha^{-2} \alpha^{2} d_{R}=d_{R} .
$$

Again, the action is trivial.

For SU(5) to work, though, $\mathbb{Z}_{6}$ has to act trivially on every fermion. There are 16 cases to check, and it is an awful lot to demand that hypercharge, the most erratic part of the Standard Model representation, satisfies 16 relations.

Or is it? In general, for a fermion with hypercharge $Y$, there are four distinct possibilities:

\begin{tabular}{llclc}
\hline \multicolumn{5}{c}{ Hypercharge relations } \\
\hline Case & Representation & & Relation \\
\hline Nontrivial SU(2), nontrivial SU(3) & $\Rightarrow$ & $\mathbb{C}_{Y} \otimes \mathbb{C}^{2} \otimes \mathbb{C}^{3}$ & $\Rightarrow$ & $\alpha^{3 Y-3+2}=1$ \\
Nontrivial SU(2), trivial SU(3) & $\Rightarrow$ & $\mathbb{C}_{Y} \otimes \mathbb{C}^{2} \otimes \mathbb{C}$ & $\Rightarrow$ & $\alpha^{3 Y-3}=1$ \\
Trivial SU(2), nontrivial SU(3) & $\Rightarrow$ & $\mathbb{C}_{Y} \otimes \mathbb{C} \otimes \mathbb{C}^{3}$ & $\Rightarrow$ & $\alpha^{3 Y+2}=1$ \\
Trivial SU(2), trivial SU(3) & $\Rightarrow$ & $\mathbb{C}_{Y} \otimes \mathbb{C} \otimes \mathbb{C}$ & $\Rightarrow$ & $\alpha^{3 Y}=1$ \\
\hline
\end{tabular}

Better yet, say it like a physicist!

\begin{tabular}{llccc}
\hline \multicolumn{4}{c}{ Hypercharge relations } \\
\hline Case & Representation & & Relation \\
\hline Left-handed quark & $\Rightarrow$ & $\mathbb{C}_{Y} \otimes \mathbb{C}^{2} \otimes \mathbb{C}^{3}$ & $\Rightarrow$ & $\alpha^{3 Y-3+2}=1$ \\
Left-handed lepton & $\Rightarrow$ & $\mathbb{C}_{Y} \otimes \mathbb{C}^{2} \otimes \mathbb{C}$ & $\Rightarrow$ & $\alpha^{3 Y-3}=1$ \\
Right-handed quark & $\Rightarrow$ & $\mathbb{C}_{Y} \otimes \mathbb{C} \otimes \mathbb{C}^{3}$ & $\Rightarrow$ & $\alpha^{3 Y+2}=1$ \\
Right-handed lepton & $\Rightarrow$ & $\mathbb{C}_{Y} \otimes \mathbb{C} \otimes \mathbb{C}$ & $\Rightarrow$ & $\alpha^{3 Y}=1$ \\
\hline
\end{tabular}

But $\alpha$ is sixth root of unity, so all this really says is that those exponents are multiples of six:

\begin{tabular}{lcc}
\hline \multicolumn{3}{c}{ Hypercharge relations } \\
\hline Case & & Relation \\
\hline Left-handed quark & $\Rightarrow$ & $3 Y-3+2 \in 6 \mathbb{Z}$ \\
Left-handed lepton & $\Rightarrow$ & $3 Y-3 \in 6 \mathbb{Z}$ \\
Right-handed quark & $\Rightarrow$ & $3 Y+2 \in 6 \mathbb{Z}$ \\
Right-handed lepton & $\Rightarrow$ & $3 Y \in 6 \mathbb{Z}$ \\
\hline
\end{tabular}


Dividing by 3 and doing a little work, it is easy to see that these are just:

TABLE 3. Hypercharge relations

\begin{tabular}{ll}
\hline \multicolumn{2}{c}{ Hypercharge relations } \\
\hline Case & Hypercharge \\
\hline Left-handed quark & $Y=$ even integer $+\frac{1}{3}$ \\
Left-handed lepton & $Y=$ odd integer \\
Right-handed quark & $Y=$ odd integer $+\frac{1}{3}$ \\
Right-handed lepton & $Y=$ even integer \\
\hline
\end{tabular}

Now it is easy to check that this indeed holds for every fermion in the standard model. SU(5) passes the test, not despite the bizarre pattern followed by hypercharges, but because of it!

By this analysis, we have shown that $\mathbb{Z}_{6}$ acts trivially on the Standard Model representation, so it is contained in the kernel of this representation. It is better than just a containment though: $\mathbb{Z}_{6}$ is the entire kernel. Because of this, we could say that $G_{\mathrm{SM}} / \mathbb{Z}_{6}$ is the "true" gauge group of the Standard Model. Also, because we now know that

$$
G_{\mathrm{SM}} / \mathbb{Z}_{6} \cong \mathrm{S}(\mathrm{U}(2) \times \mathrm{U}(3)) \hookrightarrow \mathrm{SU}(5),
$$

it is almost as though this $\mathbb{Z}_{6}$ kernel, lurking inside $G_{\mathrm{SM}}$ this whole time, was a cryptic hint to try the $\mathrm{SU}(5)$ theory.

Of course, we still need to find a representation of SU(5) that extends the Standard Model representation. Luckily, there is a very beautiful choice that works: the exterior algebra $\Lambda \mathbb{C}^{5}$. Since $\mathrm{SU}(5)$ acts on $\mathbb{C}^{5}$, it has a representation on $\Lambda \mathbb{C}^{5}$. Our next goal is to check that pulling back this representation from $\mathrm{SU}(5)$ to $G_{\mathrm{SM}}$ using $\phi$, we obtain the Standard Model representation $F \oplus F^{*}$.

As we do this, we will see another fruit of the SU(5) theory ripen. The triviality of $\mathbb{Z}_{6}$ already imposed some structure on hypercharges, as outlined above in Table 3 , As we fit the fermions into $\Lambda \mathbb{C}^{5}$, we will see this is no accident: the hypercharges have to be exactly what they are for the $\mathrm{SU}(5)$ theory to work.

To get started, our strategy will be to use the fact that, being representations of compact Lie groups, both the fermions $F \oplus F^{*}$ and the exterior algebra $\Lambda \mathbb{C}^{5}$ are completely reducible, so they can be written as a direct sum of irreps. We will then match up these irreps one at a time.

The fermions are already written as a direct sum of irreps, so we need to work on $\Lambda \mathbb{C}^{5}$. Now, any element $g \in \mathrm{SU}(5)$ acts as an automorphism of the exterior algebra $\Lambda \mathbb{C}^{5}$ :

$$
g(v \wedge w)=g v \wedge g w
$$

where $v, w \in \Lambda \mathbb{C}^{5}$. Since we know how $g$ acts on the vectors in $\mathbb{C}^{5}$, and these generate $\Lambda \mathbb{C}^{5}$, this rule is enough to tell us how $g$ acts on all of $\mathbb{C}^{5}$. This action respects grades in $\Lambda \mathbb{C}^{5}$, so each exterior power in

$$
\Lambda \mathbb{C}^{5} \cong \Lambda^{0} \mathbb{C}^{5} \oplus \Lambda^{1} \mathbb{C}^{5} \oplus \Lambda^{2} \mathbb{C}^{5} \oplus \Lambda^{3} \mathbb{C}^{5} \oplus \Lambda^{4} \mathbb{C}^{5} \oplus \Lambda^{5} \mathbb{C}^{5}
$$

is a subrepresentation. In fact, these are all irreducible, so this is how $\Lambda \mathbb{C}^{5}$ breaks up into irreps of $\mathrm{SU}(5)$. Upon restriction to $G_{\mathrm{SM}}$, some of these summands break apart further into irreps of $G_{\mathrm{SM}}$. 
Let us see how this works, starting with the easiest cases. $\Lambda^{0} \mathbb{C}^{5}$ and $\Lambda^{5} \mathbb{C}^{5}$ are both trivial irreps of $G_{\mathrm{SM}}$. There are two trivial irreps in the Standard Model representation, namely $\left\langle\nu_{R}\right\rangle$ and its dual $\left\langle\bar{\nu}_{L}\right\rangle$, where we use angle brackets to stand for the Hilbert space spanned by a vector or collection of vectors. So, we could select $\Lambda^{0} \mathbb{C}^{5}=\left\langle\bar{\nu}_{L}\right\rangle$ and $\Lambda^{5} \mathbb{C}^{5}=\left\langle\nu_{R}\right\rangle$, or vice versa. At this juncture, we have no reason to prefer one choice to the other.

Next let us chew on the next piece: the first exterior power, $\Lambda^{1} \mathbb{C}^{5}$. We have

$$
\Lambda^{1} \mathbb{C}^{5} \cong \mathbb{C}^{5}
$$

as vector spaces, and as representations of $G_{\mathrm{SM}}$. But what is $\mathbb{C}^{5}$ as a representation of $G_{\mathrm{SM}}$ ? The Standard Model gauge group acts on $\mathbb{C}^{5}$ via the map

$$
\phi:(\alpha, g, h) \longmapsto\left(\begin{array}{cc}
\alpha^{3} g & 0 \\
0 & \alpha^{-2} h
\end{array}\right) .
$$

Clearly, this action preserves the splitting into the "isospin part" and the "color part" of $\mathbb{C}^{5}$ :

$$
\mathbb{C}^{5} \cong \mathbb{C}^{2} \oplus \mathbb{C}^{3}
$$

So, let us examine these two subrepresentations in turn:

- The $\mathbb{C}^{2}$ part transforms in the hypercharge 1 representation of $U(1)$ : that is, $\alpha$ acts as multiplication by $\alpha^{3}$. It transforms according to the fundamental representation of $\mathrm{SU}(2)$, and the trivial representation of $\mathrm{SU}(3)$. This seems to describe a left-handed lepton with hypercharge 1.

- The $\mathbb{C}^{3}$ part transforms in the hypercharge $-\frac{2}{3}$ rep of $\mathrm{U}(1)$ : that is, $\alpha$ acts as multiplication by $\alpha^{-2}$. It transforms trivially under $\mathrm{SU}(2)$, and according to the fundamental representation of SU(3). Table 1 shows that these are the features of a right-handed down quark.

In short, as a representation of $G_{\mathrm{SM}}$, we have

$$
\mathbb{C}^{5} \cong \mathbb{C}_{1} \otimes \mathbb{C}^{2} \otimes \mathbb{C} \oplus \mathbb{C}_{-\frac{2}{3}} \otimes \mathbb{C} \otimes \mathbb{C}^{3},
$$

and we have already guessed which particles these correspond to. The first summand looks like a left-handed lepton with hypercharge 1, while the second is a right-handed quark with hypercharge $-\frac{2}{3}$.

Now this is problematic, because another glance at Table 1 reveals that there is no left-handed lepton with hypercharge 1 . The only particles with hypercharge 1 are the right-handed antileptons, which span the representation

$$
\left\langle\begin{array}{c}
e_{R}^{+} \\
\bar{\nu}_{R}
\end{array}\right\rangle \cong \mathbb{C}_{1} \otimes \mathbb{C}^{2 *} \otimes \mathbb{C} .
$$

But wait! $\mathrm{SU}(2)$ is unique among the $\mathrm{SU}(n)$ 's in that its fundamental representation $\mathbb{C}^{2}$ is self-dual:

$$
\mathbb{C}^{2} \cong \mathbb{C}^{2 *}
$$

This saves the day. As a representation of $G_{\mathrm{SM}}, \mathbb{C}^{5}$ becomes

$$
\mathbb{C}^{5} \cong \mathbb{C}_{1} \otimes \mathbb{C}^{2 *} \otimes \mathbb{C} \oplus \mathbb{C}_{-\frac{2}{3}} \otimes \mathbb{C} \otimes \mathbb{C}^{3},
$$

so it describes the right-handed antileptons with hypercharge 1 and the righthanded quarks with hypercharge $-\frac{2}{3}$. In other words:

$$
\Lambda^{1} \mathbb{C}^{5} \cong \mathbb{C}^{5} \cong\left\langle\begin{array}{c}
e_{R}^{+} \\
\bar{\nu}_{R}
\end{array}\right\rangle \oplus\left\langle d_{R}\right\rangle,
$$


where we have omitted the color label on $d_{R}$ to save space. Take heed of this: $\left\langle d_{R}\right\rangle$ is short for the vector space $\left\langle d_{R}^{r}, d_{R}^{g}, d_{R}^{b}\right\rangle$, and it is three-dimensional.

Now we can use our knowledge of the first exterior power to compute the second exterior power, by applying the formula

$$
\Lambda^{2}(V \oplus W) \cong \Lambda^{2} V \oplus(V \otimes W) \oplus \Lambda^{2} W .
$$

So, let us calculate! As representations of $G_{\mathrm{SM}}$ we have

$$
\begin{aligned}
\Lambda^{2} \mathbb{C}^{5} \cong & \Lambda^{2}\left(\mathbb{C}_{1} \otimes \mathbb{C}^{2} \otimes \mathbb{C} \oplus \mathbb{C}_{-\frac{2}{3}} \otimes \mathbb{C}^{2} \otimes \mathbb{C}^{3}\right) \\
\cong & \Lambda^{2}\left(\mathbb{C}_{1} \otimes \mathbb{C}^{2} \otimes \mathbb{C}\right) \oplus\left(\mathbb{C}_{1} \otimes \mathbb{C}^{2} \otimes \mathbb{C}\right) \otimes\left(\mathbb{C}_{-\frac{2}{3}} \otimes \mathbb{C} \otimes \mathbb{C}^{3}\right) \\
& \oplus \Lambda^{2}\left(\mathbb{C}_{-\frac{2}{3}} \otimes \mathbb{C} \otimes \mathbb{C}^{3}\right)
\end{aligned}
$$

Consider the first summand, $\Lambda^{2}\left(\mathbb{C}_{1} \otimes \mathbb{C}^{2} \otimes \mathbb{C}\right)$. As a representation of $\mathrm{SU}(2)$ this space is just $\Lambda^{2} \mathbb{C}^{2}$, which is the one-dimensional trivial representation, $\mathbb{C}$. As a representation of $\mathrm{SU}(3)$ it is also trivial. But as a representation of $\mathrm{U}(1)$, it is nontrivial. Inside it we are juxtaposing two particles with hypercharge 1. Hypercharges add, just like charges, so the composite particle, which consists of one particle and the other, has hypercharge 2 . So, as a representation of the Standard Model gauge group we have

$$
\Lambda^{2}\left(\mathbb{C}_{1} \otimes \mathbb{C}^{2} \otimes \mathbb{C}\right) \cong \mathbb{C}_{2} \otimes \mathbb{C} \otimes \mathbb{C}
$$

Glancing at Table 1 we see that this matches the left-handed positron, $e_{L}^{+}$. Note that the hypercharges are becoming useful now, since they uniquely identify all the fermion and antifermion representations, except for neutrinos.

Next consider the second summand:

$$
\left(\mathbb{C}_{1} \otimes \mathbb{C}^{2} \otimes \mathbb{C}\right) \otimes\left(\mathbb{C}_{-\frac{2}{3}} \otimes \mathbb{C} \otimes \mathbb{C}^{3}\right) .
$$

Again, we can add hypercharges, so this representation of $G_{\mathrm{SM}}$ is isomorphic to

$$
\mathbb{C}_{\frac{1}{3}} \otimes \mathbb{C}^{2} \otimes \mathbb{C}^{3}
$$

This is the space for left-handed quarks of hypercharge $\frac{1}{3}$, which from Table 1 is:

$$
\left\langle\begin{array}{l}
u_{L} \\
d_{L}
\end{array}\right\rangle,
$$

where once again we have suppressed the label for colors.

Finally, the third summand in $\Lambda^{2} \mathbb{C}^{5}$ is

$$
\Lambda^{2}\left(\mathbb{C}_{-\frac{2}{3}} \otimes \mathbb{C} \otimes \mathbb{C}^{3}\right)
$$

This has isospin $-\frac{4}{3}$, so by Table 1 it had better correspond to the left-handed antiup antiquark, which lives in the representation

$$
\mathbb{C}_{-\frac{4}{3}} \otimes \mathbb{C} \otimes \mathbb{C}^{3 *}
$$

Let us check. The representation $\Lambda^{2}\left(\mathbb{C}_{-\frac{2}{3}} \otimes \mathbb{C} \otimes \mathbb{C}^{3}\right)$ is trivial under $\mathrm{SU}(2)$. As a representation of $\mathrm{SU}(3)$ it is the same as $\Lambda^{2} \mathbb{C}^{3}$. But because $\mathrm{SU}(3)$ preserves the volume form on $\mathbb{C}^{3}$, taking Hodge duals gives an isomorphism

$$
\Lambda^{p} \mathbb{C}^{3} \cong\left(\Lambda^{3-p} \mathbb{C}^{3}\right)^{*}
$$

so we have

$$
\Lambda^{2} \mathbb{C}^{3} \cong\left(\Lambda^{1} \mathbb{C}^{3}\right)^{*} \cong \mathbb{C}^{3 *}
$$


which is just what we need to show

$$
\Lambda^{2}\left(\mathbb{C}_{-\frac{2}{3}} \otimes \mathbb{C}^{3}\right) \cong \mathbb{C}_{-\frac{4}{3}} \otimes \mathbb{C} \otimes \mathbb{C}^{3 *} \cong\left\langle\bar{u}_{L}\right\rangle
$$

In summary, the following pieces of the Standard Model representation sit inside $\Lambda^{2} \mathbb{C}^{5}$ :

$$
\Lambda^{2} \mathbb{C}^{5} \cong\left\langle e_{L}^{+}\right\rangle \oplus\left\langle\begin{array}{c}
u_{L} \\
d_{L}
\end{array}\right\rangle \oplus\left\langle\bar{u}_{L}\right\rangle .
$$

We are almost done. Because $\mathrm{SU}(5)$ preserves the canonical volume form on $\mathbb{C}^{5}$, taking Hodge duals gives an isomorphism between

$$
\Lambda^{p} \mathbb{C}^{5} \cong\left(\Lambda^{5-p} \mathbb{C}^{5}\right)^{*}
$$

as representations of $\mathrm{SU}(5)$. Thus given our results so far:

$$
\begin{aligned}
\Lambda^{0} \mathbb{C}^{5} \cong\left\langle\bar{\nu}_{L}\right\rangle \\
\Lambda^{1} \mathbb{C}^{5} \cong\left\langle\begin{array}{c}
e_{R}^{+} \\
\bar{\nu}_{R}
\end{array}\right\rangle \oplus\left\langle d_{R}\right\rangle, \\
\Lambda^{2} \mathbb{C}^{5} \cong\left\langle e_{L}^{+}\right\rangle \oplus\left\langle\begin{array}{c}
u_{L} \\
d_{L}
\end{array}\right\rangle \oplus\left\langle\bar{u}_{L}\right\rangle,
\end{aligned}
$$

we automatically get the antiparticles of these upon taking Hodge duals,

$$
\begin{aligned}
& \Lambda^{3} \mathbb{C}^{5} \cong\left\langle e_{R}^{-}\right\rangle \oplus\left\langle\begin{array}{c}
\bar{d}_{R} \\
\bar{u}_{R}
\end{array}\right\rangle \oplus\left\langle u_{R}\right\rangle, \\
& \Lambda^{4} \mathbb{C}^{5} \cong\left\langle\begin{array}{c}
\nu_{L} \\
e_{L}^{-}
\end{array}\right\rangle \oplus\left\langle\bar{d}_{L}\right\rangle, \\
& \Lambda^{5} \mathbb{C}^{5} \cong\left\langle\nu_{R}\right\rangle .
\end{aligned}
$$

So $\Lambda \mathbb{C}^{5} \cong F \oplus F^{*}$, as desired.

How does all this look in terms of the promised binary code? Remember, a 5-bit code is short for a wedge product of basis vectors $u, d, r, g, b \in \mathbb{C}^{5}$. For example, 01101 corresponds to $d \wedge r \wedge b$. Now that we have found an isomorphism $\Lambda \mathbb{C}^{5} \cong$ $F \oplus F^{*}$, each of these wedge products corresponds to a fermion or antifermion. How does this correspondence go, exactly?

First consider the grade-one part $\Lambda^{1} \mathbb{C}^{5} \cong \mathbb{C}^{2} \oplus \mathbb{C}^{3}$. This has basis vectors called $u, d, r, g$, and $b$. We have seen that the subspace $\mathbb{C}^{2}$, spanned by $u$ and $d$, corresponds to

$$
\left\langle\begin{array}{c}
e_{R}^{+} \\
\bar{\nu}_{R}
\end{array}\right\rangle .
$$

The top particle here has isospin up, while the bottom one has isospin down, so we must have $e_{R}^{+}=u$ and $\bar{\nu}_{R}=d$. Likewise, the subspace $\mathbb{C}^{3}$ spanned by $r, g$ and $b$ corresponds to

$$
\left\langle d_{R}\right\rangle=\left\langle d_{R}^{r}, d_{R}^{g}, d_{R}^{b}\right\rangle .
$$

Thus we must have $d_{R}^{c}=c$, where $c$ runs over the colors $r, g, b$.

Next consider the grade-two part:

$$
\Lambda^{2} \mathbb{C}^{5} \cong\left\langle e_{L}^{+}\right\rangle \oplus\left\langle\begin{array}{l}
u_{L} \\
d_{L}
\end{array}\right\rangle \oplus\left\langle\bar{u}_{L}\right\rangle .
$$

Here $e_{L}^{+}$lives in the one-dimensional $\Lambda^{2} \mathbb{C}^{2}$ representation of $\mathrm{SU}(2)$, which is spanned by the vector $u \wedge d$. Thus, $e_{L}^{+}=u \wedge d$. The left-handed quarks live in the $\mathbb{C}^{2} \otimes \mathbb{C}^{3}$ representation of $\mathrm{SU}(2) \times \mathrm{SU}(3)$, which is spanned by vectors that consist of one 
isospin and one color. We must have $u_{L}^{c}=u \wedge c$ and $d_{L}^{c}=d \wedge c$, where again $c$ runs over all the colors $r, g, b$. Now for the tricky part: the $\bar{u}_{L}$ quarks live in the $\Lambda^{2} \mathbb{C}^{3}$ representation of $\mathrm{SU}(3)$, but this is isomorphic to the fundamental representation of $\mathrm{SU}(3)$ on $\mathbb{C}^{3 *}$, which is spanned by antired, antired and antiblue:

$$
\bar{r}=g \wedge b, \quad \bar{g}=b \wedge r, \quad \bar{b}=r \wedge g .
$$

These vectors form the basis of $\Lambda^{2} \mathbb{C}^{3}$ that is dual to $r, g$, and $b$ under Hodge duality in $\Lambda \mathbb{C}^{3}$. So we must have

$$
u_{L}^{\bar{c}}=\bar{c},
$$

where $\bar{c}$ can be any anticolor. Take heed of the fact that $\bar{c}$ is grade 2 , even though it may look like grade 1 .

To work out the other grades, note that Hodge duality corresponds to switching 0's and 1's in our binary code. For instance, the dual of 01101 is 10010: or written in terms of basis vectors, the dual of $d \wedge r \wedge b$ is $u \wedge g$. Thus given the binary codes for the first few exterior powers:

\begin{tabular}{lll}
\hline$\Lambda^{0} \mathbb{C}^{5}$ & $\Lambda^{1} \mathbb{C}^{5}$ & $\Lambda^{2} \mathbb{C}^{5}$ \\
\hline $\bar{\nu}_{L}=1$ & $e_{R}^{+}=u$ & $e_{L}^{+}=u \wedge d$ \\
& $\bar{\nu}_{R}=d$ & $u_{L}^{c}=u \wedge c$ \\
& $d_{R}^{c}=c$ & $d_{L}^{c}=d \wedge c$ \\
& & $\bar{u}_{L}^{\bar{c}}=\bar{c}$ \\
\hline
\end{tabular}

taking Hodge duals gives the binary codes for the rest:

\begin{tabular}{lll}
\hline$\Lambda^{3} \mathbb{C}^{5}$ & $\Lambda^{4} \mathbb{C}^{4}$ & $\Lambda^{5} \mathbb{C}^{5}$ \\
\hline$e_{R}^{-}=r \wedge g \wedge b$ & $e_{L}^{-}=d \wedge r \wedge g \wedge b$ & $\nu_{R}=u \wedge d \wedge r \wedge g \wedge b$ \\
$\bar{u}_{R}^{\bar{c}}=d \wedge \bar{c}$ & $\nu_{L}=u \wedge r \wedge g \wedge b$ & \\
$\bar{d}_{R}^{\bar{c}}=u \wedge \bar{c}$ & $\bar{d}_{L}^{\bar{c}}=u \wedge d \wedge \bar{c}$ & \\
$u_{R}^{c}=u \wedge d \wedge c$ & & \\
\hline
\end{tabular}

Putting these together, we get the binary code for every particle and antiparticle in the first generation of fermions. To save space, let us omit the wedge product symbols:

TABLE 4. Binary code for first-generation fermions, where $c=$ $r, g, b$ and $\bar{c}=g b, b r, r g$

\begin{tabular}{cccccc}
\hline \multicolumn{5}{c}{ The Binary Code for SU(5) } \\
\hline$\Lambda^{0} \mathbb{C}^{5}$ & $\Lambda^{1} \mathbb{C}^{5}$ & $\Lambda^{2} \mathbb{C}^{5}$ & $\Lambda^{3} \mathbb{C}^{5}$ & $\Lambda^{4} \mathbb{C}^{4}$ & $\Lambda^{5} \mathbb{C}^{5}$ \\
\hline $\bar{\nu}_{L}=1$ & $e_{R}^{+}=u$ & $e_{L}^{+}=u d$ & $e_{R}^{-}=r g b$ & $e_{L}^{-}=d r g b$ & $\nu_{R}=u d r g b$ \\
& $\bar{\nu}_{R}=d$ & $u_{L}^{c}=u c$ & $\bar{u}_{R}^{c}=d \bar{c}$ & $\nu_{L}=u r g b$ & \\
& $d_{R}^{c}=c$ & $d_{L}^{c}=d c$ & $\bar{d}_{R}^{\bar{c}}=u \bar{c}$ & $\bar{d}_{L}^{\bar{c}}=u d \bar{c}$ & \\
& & $\bar{u}_{L}^{\bar{c}}=\bar{c}$ & $u_{R}^{c}=u d c$ & & \\
\hline
\end{tabular}

Now we can see a good, though not decisive, reason to choose $\Lambda^{0} \mathbb{C}^{5} \cong \bar{\nu}_{L}$. With this choice, and not the other, we get left-handed particles in the even grades, and 
right-handed particles in the odd grades. We choose to have this pattern now, but later on we need it.

Table 4 defines a linear isomorphism $f: F \oplus F^{*} \rightarrow \Lambda \mathbb{C}^{5}$ in terms of the basis vectors, so the equations in this table are a bit of an exaggeration. When we write, say, $e_{R}^{+}=u$, we really mean $f\left(e_{R}^{+}\right)=u$. This map $f$ is an isomorphism between representations of $G_{\mathrm{SM}}$. It tells us how these representations are the "same".

More precisely, we mean that these representations are the same when we identify $\mathrm{S}(\mathrm{U}(2) \times \mathrm{U}(3))$ with $G_{\mathrm{SM}} / \mathbb{Z}_{6}$ using the isomorphism induced by $\phi$. In general, we can think of a unitary representation as a Lie group homomorphism

$$
G \rightarrow \mathrm{U}(V)
$$

where $V$ is a finite-dimensional Hilbert space and $\mathrm{U}(V)$ is the Lie group of unitary operators on $V$. In this section we have been comparing two unitary representations: an ugly, complicated representation of $G_{\mathrm{SM}}$ :

$$
G_{\mathrm{SM}} \rightarrow \mathrm{U}\left(F \oplus F^{*}\right)
$$

and a nice, beautiful representation of $\mathrm{SU}(5)$ :

$$
\mathrm{SU}(5) \rightarrow \mathrm{U}\left(\Lambda \mathbb{C}^{5}\right)
$$

We built a homomorphism

$$
\phi: G_{\mathrm{SM}} \rightarrow \mathrm{SU}(5)
$$

so it is natural to wonder if is there a fourth homomorphism

$$
\mathrm{U}\left(F \oplus F^{*}\right) \rightarrow \mathrm{U}\left(\Lambda \mathbb{C}^{5}\right)
$$

such that this square:

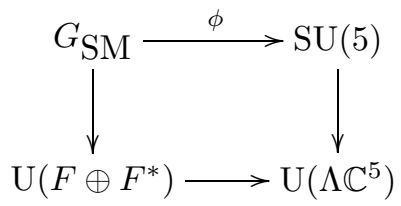

commutes.

Indeed, we just showed this! We have seen that there exists a unitary operator from the Standard Model representation to $\Lambda \mathbb{C}^{5}$, say

$$
f: F \oplus F^{*} \stackrel{\sim}{\rightarrow} \Lambda \mathbb{C}^{5},
$$

such that the induced isomorphism of the unitary groups,

$$
\mathrm{U}(f): \mathrm{U}\left(F \oplus F^{*}\right) \stackrel{\sim}{\rightarrow} \mathrm{U}\left(\Lambda \mathbb{C}^{5}\right),
$$

makes the above square commute. So, let us summarize this result as a theorem:

Theorem 1. The following square commutes:

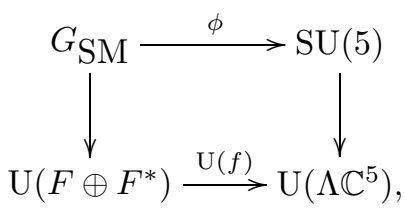

where the left vertical arrow is the Standard Model representation and the right one is the natural representation of $\mathrm{SU}(5)$ on the exterior algebra of $\mathbb{C}^{5}$. 
3.2. The Spin(10) theory. We now turn our attention to another grand unified theory. Physicists call it the "SO(10) theory", but we shall call it the $\operatorname{Spin}(10)$ theory, because the Lie group involved is really Spin(10), the double cover of $\mathrm{SO}(10)$. This theory appeared in a 1974 paper by Georgi [10, shortly after his paper with Glashow on the SU(5) theory. However, Georgi has said that he conceived of the Spin(10) theory first. See Zee [40, Chapter VII.7, for a concise and readable account.

The SU(5) GUT has helped us explain the pattern of hypercharges in the Standard Model, and thanks to the use of the exterior algebra, $\Lambda \mathbb{C}^{5}$, we can interpret it in terms of a binary code. This binary code explains another curious fact about the Standard Model. Specifically, why is the number of fermions a power of 2? There are 16 fermions, and 16 antifermions, which makes the Standard Model representation have dimension

$$
\operatorname{dim}\left(F \oplus F^{*}\right)=2^{5}=32 .
$$

With the binary code interpretation, it could not be any other way.

In actuality, however, the existence of a right-handed neutrino (or its antiparticle, the left-handed antineutrino) has been controversial. Because it transforms trivially in the Standard Model, it does not interact with anything except perhaps the Higgs.

The right-handed neutrino certainly improves the aesthetics of the SU(5) theory. When we include this particle (and its antiparticle), we obtain the representation

$$
\Lambda^{0} \mathbb{C}^{5} \oplus \Lambda^{1} \mathbb{C}^{5} \oplus \Lambda^{2} \mathbb{C}^{5} \oplus \Lambda^{3} \mathbb{C}^{5} \oplus \Lambda^{4} \mathbb{C}^{5} \oplus \Lambda^{5} \mathbb{C}^{5},
$$

which is all of $\Lambda \mathbb{C}^{5}$, whereas without this particle we would just have

$$
\Lambda^{1} \mathbb{C}^{5} \oplus \Lambda^{2} \mathbb{C}^{5} \oplus \Lambda^{3} \mathbb{C}^{5} \oplus \Lambda^{4} \mathbb{C}^{5},
$$

which is much less appealing; it wants to be $\Lambda \mathbb{C}^{5}$, but it comes up short.

More importantly, there is increasing indirect evidence from experimental particle physics that right-handed neutrinos $d o$ exist. For details, see Pati [27]. If this is true, the number of fermions really could be 16 , and we have a ready-made explanation for that number in the binary code.

However, this creates a new mystery. The SU(5) works nicely with the representation $\Lambda \mathbb{C}^{5}$, but $\mathrm{SU}(5)$ does not require this. It works just fine with the smaller representation

$$
\Lambda^{1} \mathbb{C}^{5} \oplus \Lambda^{2} \mathbb{C}^{5} \oplus \Lambda^{3} \mathbb{C}^{5} \oplus \Lambda^{4} \mathbb{C}^{5} .
$$

It would be nicer to have a theory that required us to use all of $\Lambda \mathbb{C}^{5}$. Better yet, if our new GUT were an extension of SU(5), the beautiful explanation of hypercharges would live on in our new theory. With luck, we might even get away with using the same underlying vector space, $\Lambda \mathbb{C}^{5}$. Could it be that the $\mathrm{SU}(5)$ GUT is only the beginning of the story? Could unification go on, with a grand unified theory that extends SU(5) just as SU(5) extended the Standard Model?

Let us look for a group that extends $\mathrm{SU}(5)$ and has an irrep whose dimension is some power of 2 . The dimension is a big clue. What representations have dimensions that are powers of 2? Spinors.

What are spinors? They are certain representations of $\operatorname{Spin}(n)$, the double cover of the rotation group in $n$ dimensions, which do not factor through the quotient $\mathrm{SO}(n)$. Their dimensions are always a power of two. We build them by exhibiting $\operatorname{Spin}(n)$ as a subgroup of a Clifford algebra. Recall that the Clifford algebra 
Cliff $_{n}$ is the associative algebra freely generated by $\mathbb{R}^{n}$ with relations

$$
v w+w v=-2\langle v, w\rangle .
$$

If we take products of pairs of unit vectors in $\mathbb{R}^{n}$ inside this algebra, these generate the group $\operatorname{Spin}(n)$ : multiplication in this group coincides with multiplication in the Clifford algebra. Using this fact, we can get representations of $\operatorname{Spin}(2 n)$ from modules of Cliff ${ }_{n}$.

We can use this method to get a representation of $\operatorname{Spin}(10)$ on $\Lambda \mathbb{C}^{5}$ that extends the representation of $\mathrm{SU}(5)$ on this space. In fact, quite generally, $\mathrm{Cliff}_{2 n}$ acts on $\Lambda \mathbb{C}^{n}$. Then, because

$$
\operatorname{Spin}(2 n) \hookrightarrow \operatorname{Cliff}_{2 n},
$$

$\Lambda \mathbb{C}^{n}$ becomes a representation of $\operatorname{Spin}(2 n)$, called the Dirac spinor representation.

To see this, we use operators on $\Lambda \mathbb{C}^{n}$ called "creation and annihilation operators". Let $e_{1}, \ldots, e_{n}$ be the standard basis for $\mathbb{C}^{n}$. Each of these gives a creation operator:

$$
\begin{aligned}
a_{j}^{*}: \Lambda \mathbb{C}^{n} & \rightarrow \Lambda \mathbb{C}^{n} \\
\psi & \mapsto e_{j} \wedge \psi .
\end{aligned}
$$

We use the notation $a_{j}^{*}$ because $\Lambda \mathbb{C}^{n}$ is a Hilbert space, so $a_{j}^{*}$ is the adjoint of some other operator

$$
a_{j}: \Lambda \mathbb{C}^{n} \rightarrow \Lambda \mathbb{C}^{n},
$$

which is called an annihilation operator.

In physics, we can think of the basis vectors $e_{j}$ as particles. For example, in the binary code approach to the SU(5) theory we imagine five particles from which the observed particles in the Standard Model are composed: up, down, red, green and blue. Taking the wedge product with $e_{j}$ "creates a particle" of type $j$, while the adjoint "annihilates a particle" of type $j$.

It may seem odd that creation is the adjoint of annihilation, rather than its inverse. One reason for this is that the creation operator, $a_{j}^{*}$, has no inverse. In some sense, its adjoint $a_{j}$ is the best substitute.

This adjoint does do what want, which is to delete any particle of type $j$. Explicitly, it deletes the "first" occurrence of $e_{j}$ from any basis element, bringing out any minus signs we need to make this respect the antisymmetry of the wedge product:

$$
a_{j}\left(e_{i_{1}} \wedge \cdots \wedge e_{i_{p}}\right)=(-1)^{k+1} e_{i_{1}} \wedge \cdots \wedge e_{i_{k-1}} \wedge e_{i_{k+1}} \cdots \wedge e_{i_{p}} \text { if } j=i_{k} .
$$

If no particle of type $j$ appears, we get zero.

Now, whenever we have an inner product space such as $\mathbb{C}^{n}$, we get an inner product on $\Lambda \mathbb{C}^{n}$. The fastest, if not most elegant, route to this inner product is to remember that, given an orthonormal basis $e_{1}, \ldots, e_{n}$ for $\mathbb{C}^{n}$, the induced basis, consisting of elements of the form $e_{i_{1}} \wedge \cdots \wedge e_{i_{p}}$, should be orthonormal in $\Lambda \mathbb{C}^{n}$. But choosing an orthonormal basis defines an inner product, and in this case it defines an inner product on the whole exterior algebra, one that reduces to the usual one for the grade one elements, $\Lambda^{1} \mathbb{C}^{n} \cong \mathbb{C}^{n}$.

It is with respect to this inner product on $\Lambda \mathbb{C}^{n}$ that $a_{j}$ and $a_{j}^{*}$ are adjoint. That is, they satisfy

$$
\left\langle v, a_{j} w\right\rangle=\left\langle a_{j}^{*} v, w\right\rangle
$$

for any elements $v, w \in \Lambda \mathbb{C}^{n}$. Showing this from the definitions we have given is a straightforward calculation, which we leave to the reader. 
These operators satisfy the following relations:

$$
\begin{aligned}
& \left\{a_{j}, a_{k}\right\}=0, \\
& \left\{a_{j}^{*}, a_{k}^{*}\right\}=0, \\
& \left\{a_{j}, a_{k}^{*}\right\}=\delta_{j k},
\end{aligned}
$$

where curly brackets denote the anticommutator of two linear operators, namely $\{a, b\}=a b+b a$.

As an algebra, Cliff ${ }_{2 n}$ is generated by the standard basis vectors of $\mathbb{R}^{2 n}$. Let us call the elements of Cliff $_{2 n}$ corresponding to these basis vectors $\gamma_{1}, \ldots, \gamma_{2 n}$. From the definition of the Clifford algebra, it is easy to check that

$$
\left\{\gamma_{k}, \gamma_{\ell}\right\}=-2 \delta_{k \ell}
$$

In other words, the elements $\gamma_{k}$ are anticommuting square roots of -1 . So, we can turn $\Lambda \mathbb{C}^{n}$ into a Cliff $2 n$-module by finding $2 n$ linear operators on $\Lambda \mathbb{C}^{n}$ that anticommute and square to -1 . We build these from the raw material provided by $a_{j}$ and $a_{j}^{*}$. Indeed, it is easy to see that

$$
\begin{aligned}
& \phi_{j}=i\left(a_{j}+a_{j}^{*}\right), \\
& \pi_{j}=a_{j}-a_{j}^{*}
\end{aligned}
$$

do the trick. Now we can map $\gamma_{1}, \ldots, \gamma_{2 n}$ to these operators, in any order, and

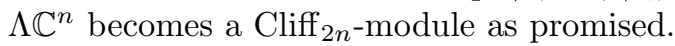

Now for $n>1$ we may define $\operatorname{Spin}(2 n)$ to be the universal cover of $\operatorname{SO}(2 n)$, with group structure making the covering map

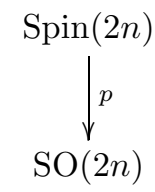

into a homomorphism. This universal cover is a double cover, because the fundamental group of $\mathrm{SO}(2 n)$ is $\mathbb{Z}_{2}$ for $n$ in this range.

This construction of $\operatorname{Spin}(2 n)$ is fairly abstract. Luckily, we can realize $\operatorname{Spin}(2 n)$ as the multiplicative group in Cliff $_{2 n}$ generated by products of pairs of unit vectors. This gives us the inclusion

$$
\operatorname{Spin}(2 n) \hookrightarrow \operatorname{Cliff}_{2 n}
$$

that we need to make $\Lambda \mathbb{C}^{n}$ into a representation of $\operatorname{Spin}(2 n)$. From this, one can show that the Lie algebra $\mathfrak{s o}(2 n)$ is generated by the commutators of the $\gamma_{j}$. Because we know how to map each $\gamma_{j}$ to an operator on $\Lambda \mathbb{C}^{n}$, this gives us an explicit formula for the action of $\mathfrak{s o}(2 n)$ on $\Lambda \mathbb{C}^{n}$. Each $\gamma_{j}$ changes the parity of the grades, and their commutators do this twice, restoring grade parity. Thus, $\mathfrak{s o}(2 n)$ preserves the parity of the grading on $\Lambda \mathbb{C}^{n}$, and $\operatorname{Spin}(2 n)$ does the same. This breaks $\Lambda \mathbb{C}^{n}$ into two subrepresentations:

$$
\Lambda \mathbb{C}^{n}=\Lambda^{\mathrm{ev}} \mathbb{C}^{n} \oplus \Lambda^{\text {odd }} \mathbb{C}^{n},
$$

where $\Lambda^{\mathrm{ev}} \mathbb{C}^{n}$ is the direct sum of the even-graded parts:

$$
\Lambda^{\mathrm{ev}} \mathbb{C}^{n}=\Lambda^{0} \mathbb{C}^{n} \oplus \Lambda^{2} \mathbb{C}^{n} \oplus \cdots
$$

while $\Lambda^{\text {odd }} \mathbb{C}^{n}$ is the sum of the odd-graded parts:

$$
\Lambda^{\text {odd }} \mathbb{C}^{n}=\Lambda^{1} \mathbb{C}^{n} \oplus \Lambda^{3} \mathbb{C}^{n} \oplus \cdots
$$


In fact, both these representations of $\operatorname{Spin}(2 n)$ are irreducible, and $\operatorname{Spin}(2 n)$ acts faithfully on their direct sum $\Lambda \mathbb{C}^{n}$. Elements of these two irreps of $\operatorname{Spin}(2 n)$ are called left- and right-handed Weyl spinors, respectively, while elements of $\Lambda \mathbb{C}^{n}$ are called Dirac spinors.

All this works for any $n$, but we are especially interested in the case $n=5$. The big question is: does the Dirac spinor representation of Spin(10) extend the obvious representation of $\mathrm{SU}(5)$ on $\Lambda \mathbb{C}^{5}$ ? Or, more generally, does the Dirac spinor representation of $\operatorname{Spin}(2 n)$ extend the representation of $\mathrm{SU}(n)$ on $\Lambda \mathbb{C}^{n}$ ?

Remember, we can think of a unitary representation as a group homomorphism

$$
G \rightarrow \mathrm{U}(V)
$$

where $V$ is the Hilbert space on which $G$ acts as unitary operators. Here we are concerned with two representations. One of them is the familiar representation of $\mathrm{SU}(n)$ on $\Lambda \mathbb{C}^{n}$ :

$$
\rho: \mathrm{SU}(n) \rightarrow \mathrm{U}\left(\Lambda \mathbb{C}^{n}\right),
$$

which acts as the fundamental representation on $\Lambda^{1} \mathbb{C}^{n} \cong \mathbb{C}^{n}$ and respects wedge products. The other is the representation of $\operatorname{Spin}(2 n)$ on the Dirac spinors, which happen to form the same vector space $\Lambda \mathbb{C}^{n}$ :

$$
\rho^{\prime}: \operatorname{Spin}(2 n) \rightarrow \mathrm{U}\left(\Lambda \mathbb{C}^{n}\right) .
$$

Our big question is answered affirmatively by this theorem, which can be found in a classic paper by Atiyah, Bott and Shapiro [2]:

Theorem 2. There exists a Lie group homomorphism $\psi$ that makes this triangle commute:

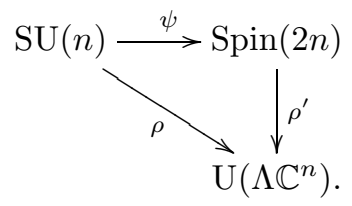

Proof. The complex vector space $\mathbb{C}^{n}$ has an underlying real vector space of dimension $2 n$, and the real part of the usual inner product on $\mathbb{C}^{n}$ gives an inner product on this underlying real vector space, so we have an inclusion $\mathrm{U}(n) \hookrightarrow \mathrm{O}(2 n)$. The connected component of the identity in $\mathrm{O}(2 n)$ is $\mathrm{SO}(2 n)$, and $\mathrm{U}(n)$ is connected, so this gives an inclusion $\mathrm{U}(n) \hookrightarrow \mathrm{SO}(2 n)$ and thus $\mathrm{SU}(n) \hookrightarrow \mathrm{SO}(2 n)$. Passing to Lie algebras, we obtain an inclusion $\mathfrak{s u}(n) \hookrightarrow \mathfrak{s o}(2 n)$. A homomorphism of Lie algebras gives a homomorphism of the corresponding simply connected Lie groups, so this in turn gives the desired map $\psi: \mathrm{SU}(n) \rightarrow \operatorname{Spin}(2 n)$.

Next we must check that $\psi$ makes the above triangle commute. Since all the groups involved are connected, it suffices to check that this diagram

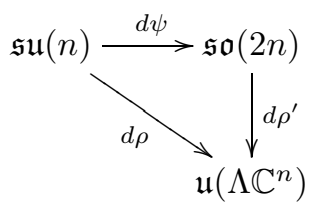

commutes. Since the Dirac representation $d \rho^{\prime}$ is defined in terms of creation and annihilation operators, we should try to express $d \rho$ this way. To do so, we will need 
a good basis for $\mathfrak{s u}(n)$. Remember,

$$
\mathfrak{s u}(n)=\{n \times n \text { traceless skew-adjoint matrices over } \mathbb{C}\} .
$$

If $E_{j k}$ denotes the matrix with 1 in the $j k$ th entry and 0 everywhere else, then the traceless skew-adjoint matrices have this basis:

$$
\begin{array}{ll}
E_{j k}-E_{k j} & j>k, \\
i\left(E_{j k}+E_{k j}\right) & j>k, \\
i\left(E_{j j}-E_{j+1, j+1}\right) & j=1, \ldots, n-1 .
\end{array}
$$

For example, $\mathfrak{s u}(2)$ has the basis

$$
\left(\begin{array}{rr}
0 & -1 \\
1 & 0
\end{array}\right), \quad\left(\begin{array}{rr}
0 & i \\
i & 0
\end{array}\right), \quad\left(\begin{array}{rr}
i & 0 \\
0 & -i
\end{array}\right),
$$

and our basis for $\mathfrak{s u}(n)$ simply generalizes this.

Now, it is easy to guess a formula for $d \rho$ in terms of creation and annihilation operators. After all, the elementary matrix $E_{j k}$ satisfies

$$
E_{j k}\left(e_{\ell}\right)=\left\{\begin{array}{cl}
e_{j} & \text { if } \ell=k \\
0 & \text { if } \ell \neq k
\end{array}\right.
$$

and $a_{j}^{*} a_{k}$ acts the same way on $\Lambda^{1} \mathbb{C}^{n} \subseteq \Lambda \mathbb{C}^{n}$. So, we certainly have

$$
\begin{aligned}
d \rho\left(E_{j k}-E_{k j}\right) & =a_{j}^{*} a_{k}-a_{k}^{*} a_{j} \\
d \rho\left(i\left(E_{j k}+E_{k j}\right)\right) & =i\left(a_{j}^{*} a_{k}+a_{k}^{*} a_{j}\right) \\
d \rho\left(i\left(E_{j j}-E_{j+1, j+1}\right)\right) & =i\left(a_{j}^{*} a_{j}-a_{j+1}^{*} a_{j+1}\right)
\end{aligned}
$$

on the subspace $\Lambda^{1} \mathbb{C}^{n}$. But do these operators agree on the rest of $\Lambda \mathbb{C}^{n}$ ? Remember, $\rho$ preserves wedge products:

$$
\rho(x)(v \wedge w)=\rho(x) v \wedge \rho(x) w
$$

for all $x \in \mathrm{SU}(n)$. Differentiating this condition, we see that $\mathfrak{s u}(n)$ must act as derivations:

$$
d \rho(X)(v \wedge w)=d \rho(X) v \wedge w+v \wedge d \rho(X) w
$$

for all $X \in \mathfrak{s u}(n)$. Derivations of $\Lambda \mathbb{C}^{n}$ are determined by their action on $\Lambda^{1} \mathbb{C}^{n}$. So, $d \rho$ will be given on all of $\Lambda \mathbb{C}^{n}$ by the above formulas if we can show that

$$
a_{j}^{*} a_{k}-a_{k}^{*} a_{j}, \quad i\left(a_{j}^{*} a_{k}+a_{k}^{*} a_{j}\right), \quad \text { and } \quad i\left(a_{j}^{*} a_{j}-a_{j+1}^{*} a_{j+1}\right)
$$

are derivations.

Now, the annihilation operators are a lot like derivations: they are antiderivations. That is, if $v \in \Lambda^{p} \mathbb{C}^{n}$ and $w \in \Lambda^{q} \mathbb{C}^{n}$, then

$$
a_{j}(v \wedge w)=a_{j} v \wedge w+(-1)^{p} v \wedge a_{j} w .
$$

However, the creation operators are nothing like derivations. They satisfy

$$
a_{j}^{*}(v \wedge w)=a_{j}^{*} v \wedge w=(-1)^{p} v \wedge a_{j}^{*} w,
$$

because $a_{j}^{*}$ acts by wedging with $e_{j}$, and moving this through $v$ introduces $p$ minus signs. Luckily, this relation combines with the previous one to make the composites $a_{j}^{*} a_{k}$ into derivations for every $j$ and $k$. We leave this for the reader to check. 
So, $d \rho$ can really be expressed in terms of annihilation and creation operators as above. Checking that

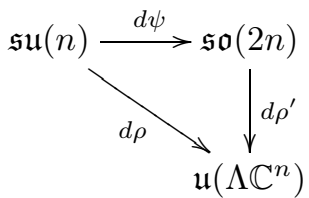

commutes is now a straightforward but somewhat tedious job, which we leave to the dedicated reader.

This theorem had a counterpart for the SU(5) GUT, namely, Theorem 1. There we saw a homomorphism $\phi$ that showed us how to extend the Standard Model group $G_{\mathrm{SM}}$ to $\mathrm{SU}(5)$, and made this square commute:

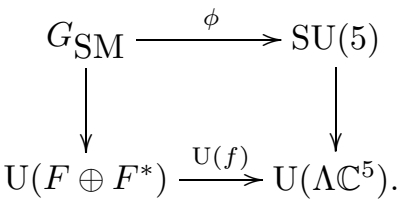

Now $\psi$ says how to extend $\mathrm{SU}(5)$ further to $\mathrm{Spin}(10)$, and makes this square commute:

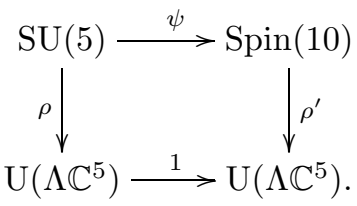

We can put these squares together to get this commutative diagram:

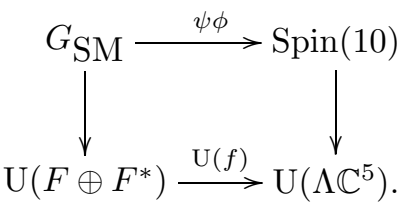

This diagram simply says that $\operatorname{Spin}(10)$ is a GUT: it extends the Standard Model group $G_{\mathrm{SM}}$ in a way that is compatible with the Standard Model representation, $F \oplus F^{*}$. In Section 3.1, all the hard work lay in showing that the representations $F \oplus F^{*}$ and $\Lambda \mathbb{C}^{5}$ of $G_{\mathrm{SM}}$ were the same. Here, we do not have to do that. We just showed that $\operatorname{Spin}(10)$ extends $\mathrm{SU}(5)$. Since $\mathrm{SU}(5)$ already extended $G_{\mathrm{SM}}$, $\mathrm{Spin}(10)$ extends that, too.

3.3. The Pati-Salam model. Next we turn to a unified theory that is not so "grand": its gauge group is not a simple Lie group, as it was for the SU(5) and Spin(10) theories. This theory is called the Pati-Salam model, after its inventors [28]; it has gauge group $\mathrm{SU}(2) \times \mathrm{SU}(2) \times \mathrm{SU}(4)$, which is merely semisimple.

We might imagine the $\mathrm{SU}(5)$ theory as an answer to this question:

Why are the hypercharges in the Standard Model what they are?

The answer it provides is something like this:

Because $\mathrm{SU}(5)$ is the actual gauge group of the world, acting on the representation $\Lambda \mathbb{C}^{5}$. 
But there are other intriguing patterns in the Standard Model that SU(5) does not explain, and these lead us in different directions.

First, there is a strange similarity between quarks and leptons. Each generation of fermions in the Standard Model has two quarks and two leptons. For example, in the first generation we have the quarks $u$ and $d$, and the leptons $\nu$ and $e^{-}$. The quarks come in three "colors": this is a picturesque way of saying that they transform in the fundamental representation of $\mathrm{SU}(3)$ on $\mathbb{C}^{3}$. The leptons, on the other hand, are "white": they transform in the trivial representation of $\mathrm{SU}(3)$ on $\mathbb{C}$.

\begin{tabular}{lc}
\hline \multicolumn{2}{l}{ Representations of $\mathbf{S U}(\mathbf{3})$} \\
\hline Particle & Representation \\
\hline Quark & $\mathbb{C}^{3}$ \\
Lepton & $\mathbb{C}$
\end{tabular}

Could the lepton secretly be a fourth color of quark? Maybe it could in a theory where the $\mathrm{SU}(3)$ color symmetry of the Standard Model is extended to $\mathrm{SU}(4)$. Of course this larger symmetry would need to be broken to explain the very real difference between leptons and quarks.

Second, there is a strange difference between left- and right-handed fermions. The left-handed ones participate in the weak force governed by SU(2), while the right-handed ones do not. Mathematically speaking, the left-handed ones live in a nontrivial representation of $\mathrm{SU}(2)$, while the right-handed ones live in a trivial one. The nontrivial one is $\mathbb{C}^{2}$, while the trivial one is $\mathbb{C} \oplus \mathbb{C}$ :

\begin{tabular}{lc}
\hline \multicolumn{2}{c}{ Representations of SU(2) } \\
\hline Particle & Representation \\
\hline Left-handed fermion & $\mathbb{C}^{2}$ \\
Right-handed fermion & $\mathbb{C} \oplus \mathbb{C}$ \\
\hline
\end{tabular}

But there is a suspicious similarity between $\mathbb{C}^{2}$ and $\mathbb{C} \oplus \mathbb{C}$. Could there be another copy of $\mathrm{SU}(2)$ that acts on the right-handed particles? Again, this "right-handed" $\mathrm{SU}(2)$ would need to be broken, to explain why we do not see a "right-handed" version of the weak force that acts on right-handed particles.

Following Pati and Salam, let us try to sculpt a theory that makes these ideas precise. In the last two sections, we saw some of the ingredients we need to make a grand unified theory: we need to extend the symmetry group $G_{\mathrm{SM}}$ to a larger group $G$ using an inclusion

$$
G_{\mathrm{SM}} \hookrightarrow G
$$

(up to some discrete kernel), and we need a representation $V$ of $G$ which reduces to the Standard Model representation when restricted to $G_{\mathrm{SM}}$ :

$$
F \oplus F^{*} \cong V .
$$

We can put all these ingredients together into a diagram

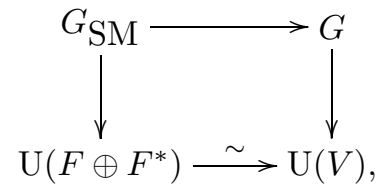

which commutes only when our $G$ theory works out. 
We now use the same methods to chip away at our current challenge. We asked if leptons correspond to a fourth color. We already know that every quark comes in three colors, $r, g$, and $b$, which form a basis for the vector space $\mathbb{C}^{3}$. This is the fundamental representation of $\mathrm{SU}(3)$, the color symmetry group of the Standard Model. If leptons correspond to a fourth color, say "white", then we should use the colors $r, g, b$ and $w$, as a basis for the vector space $\mathbb{C}^{4}$. This is the fundamental representation of $\mathrm{SU}(4)$, so let us take that group to describe color symmetries in our new GUT.

Now SU(3) has an obvious inclusion into SU(4), using block diagonal matrices:

$$
g \mapsto\left(\begin{array}{cc}
g & 0 \\
0 & 1
\end{array}\right) .
$$

When restricted to this subgroup, the fundamental representation $\mathbb{C}^{4}$ breaks into a direct sum of irreps:

$$
\mathbb{C}^{4} \cong \mathbb{C}^{3} \oplus \mathbb{C}
$$

These are precisely the irreps of $\mathrm{SU}(3)$ that describe quarks and leptons. For antiquarks and antileptons we can use

$$
\mathbb{C}^{4 *} \cong \mathbb{C}^{3 *} \oplus \mathbb{C} .
$$

It looks like we are on the right track.

We can do even better if we start with the splitting

$$
\mathbb{C}^{4} \cong \mathbb{C}^{3} \oplus \mathbb{C}
$$

Remember that when we studied $\mathrm{SU}(5)$, the splitting

$$
\mathbb{C}^{5} \cong \mathbb{C}^{2} \oplus \mathbb{C}^{3}
$$

had the remarkable effect of introducing U(1), and thus hypercharge, into SU(5) theory. This was because the subgroup of $\mathrm{SU}(5)$ that preserves this splitting is larger than $\mathrm{SU}(2) \times \mathrm{SU}(3)$, roughly by a factor of $\mathrm{U}(1)$ :

$$
(\mathrm{U}(1) \times \mathrm{SU}(2) \times \mathrm{SU}(3)) / \mathbb{Z}_{6} \cong \mathrm{S}(\mathrm{U}(2) \times \mathrm{U}(3)) .
$$

It was this factor of $\mathrm{U}(1)$ that made $\mathrm{SU}(5)$ theory so fruitful.

So, if we choose a splitting $\mathbb{C}^{4} \cong \mathbb{C}^{3} \oplus \mathbb{C}$, we should again look at the subgroup that preserves this splitting, Namely,

$$
\mathrm{S}(\mathrm{U}(3) \times \mathrm{U}(1)) \subseteq \mathrm{SU}(4) .
$$

Just as in the $\mathrm{SU}(5)$ case, this group is bigger than $\mathrm{SU}(3) \times \mathrm{SU}(1)$, roughly by a factor of $\mathrm{U}(1)$, and again, this factor of $\mathrm{U}(1)$ is related to hypercharge!

This works very much as it did for $\mathrm{SU}(5)$. We want a map

$$
\mathrm{U}(1) \times \mathrm{SU}(3) \rightarrow \mathrm{SU}(4)
$$

and we already have one that works for the $\mathrm{SU}(3)$ part:

$$
\begin{aligned}
& \mathrm{SU}(3) \rightarrow \quad \mathrm{SU}(4) \\
& h \mapsto\left(\begin{array}{cc}
h & 0 \\
0 & 1
\end{array}\right) .
\end{aligned}
$$

So, we just need to include a factor of $\mathrm{U}(1)$ that commutes with everything in the $\mathrm{SU}(3)$ subgroup. Elements of $\mathrm{SU}(4)$ that do this are of the form

$$
\left(\begin{array}{ll}
\alpha & 0 \\
0 & \beta
\end{array}\right)
$$


where $\alpha$ stands for the $3 \times 3$ identity matrix times the complex number $\alpha \in \mathrm{U}(1)$, and similarly for $\beta$ in the $1 \times 1$ block. For the above matrix to lie in $\mathrm{SU}(4)$, it must have determinant 1 , so $\alpha^{3} \beta=1$. Thus we must include $\mathrm{U}(1)$ using matrices of this form:

$$
\left(\begin{array}{cc}
\alpha & 0 \\
0 & \alpha^{-3}
\end{array}\right)
$$

This gives our map:

$$
\begin{array}{clc}
\mathrm{U}(1) \times \mathrm{SU}(3) & \rightarrow & \mathrm{SU}(4) \\
(\alpha, h) & \mapsto & \left(\begin{array}{cc}
\alpha h & 0 \\
0 & \alpha^{-3}
\end{array}\right) .
\end{array}
$$

If we let $\mathrm{U}(1) \times \mathrm{SU}(3)$ act on $\mathbb{C}^{4} \cong \mathbb{C}^{3} \oplus \mathbb{C}$ via this map, the "quark part" $\mathbb{C}^{3}$ transforms as though it has hypercharge $\frac{1}{3}$ : that is, it gets multiplied by a factor of $\alpha$. Meanwhile, the "lepton part" $\mathbb{C}$ transforms as though it has hypercharge -1 , getting multiplied by a factor of $\alpha^{-3}$. So, as a representation of $\mathrm{U}(1) \times \mathrm{SU}(3)$, we have

$$
\mathbb{C}^{4} \cong \mathbb{C}_{\frac{1}{3}} \otimes \mathbb{C}^{3} \oplus \quad \mathbb{C}_{-1} \otimes \mathbb{C}
$$

A peek at Table 1 reveals something nice. This is exactly how the left-handed quarks and leptons in the Standard Model transform under $\mathrm{U}(1) \times \mathrm{SU}(3)$ !

The right-handed leptons do not work this way. That is a problem we need to address. But this brings us to our second question, which was about the strange difference between left- and right-handed particles.

Remember that in the Standard Model, the left-handed particles live in the fundamental representation of $\mathrm{SU}(2)$ on $\mathbb{C}^{2}$, while the right-handed ones live in the trivial representation on $\mathbb{C} \oplus \mathbb{C}$. Physicists write this by grouping left-handed particles into "doublets", while leaving the right-handed particles as "singlets":

$$
\left(\begin{array}{c}
\nu_{L} \\
e_{L}^{-}
\end{array}\right) \quad e_{R}^{-} .
$$

But there is a suspicious similarity between $\mathbb{C}^{2}$ and $\mathbb{C} \oplus \mathbb{C}$. Could there be another copy of $\mathrm{SU}(2)$ that acts on the right-handed particles? Physically speaking, this would mean that the left- and right-handed particles both form doublets:

$$
\left(\begin{array}{c}
\nu_{L} \\
e_{L}^{-}
\end{array}\right) \quad\left(\begin{array}{c}
\nu_{R} \\
e_{R}^{-}
\end{array}\right)
$$

but under the actions of different SU(2)'s. Mathematically, this would amount to extending the representations of the "left-handed" $\mathrm{SU}(2)$ :

$$
\mathbb{C}^{2} \quad \mathbb{C} \oplus \mathbb{C}
$$

to representations of $\mathrm{SU}(2) \times \mathrm{SU}(2)$ :

$$
\mathbb{C}^{2} \otimes \mathbb{C} \quad \mathbb{C} \otimes \mathbb{C}^{2},
$$

where the first copy of $\mathrm{SU}(2)$ acts on the first factor in these tensor products, while the second copy acts on the second factor. The first copy of SU(2) is the "left-handed" one familiar from the Standard Model. The second copy is a new "right-handed" one. 
If we restrict these representations to the "left-handed" $\mathrm{SU}(2)$ subgroup, we obtain:

$$
\begin{aligned}
& \mathbb{C}^{2} \otimes \mathbb{C} \cong \mathbb{C}^{2} \\
& \mathbb{C} \otimes \mathbb{C}^{2} \cong \mathbb{C} \oplus \mathbb{C} .
\end{aligned}
$$

These are exactly the representations of SU(2) that appear in the Standard Model. It looks like we are on the right track!

Now let us try to combine these ideas into a theory with symmetry group $\mathrm{SU}(2) \times$ $\mathrm{SU}(2) \times \mathrm{SU}(4)$. We have seen that letting $\mathrm{SU}(4)$ act on $\mathbb{C}^{4}$ is a good way to unify our treatment of color for all the left-handed fermions. Similarly, the dual representation on $\mathbb{C}^{4 *}$ is good for their antiparticles. So, we will tackle color by letting $\mathrm{SU}(4)$ act on the direct sum $\mathbb{C}^{4} \oplus \mathbb{C}^{4 *}$. This space is eight dimensional. We have also seen that letting $\mathrm{SU}(2) \times \mathrm{SU}(2)$ act on $\mathbb{C}^{2} \otimes \mathbb{C} \oplus \mathbb{C} \otimes \mathbb{C}^{2}$ is a good way to unify our treatment of isospin for left- and right-handed fermions. This space is four dimensional.

Since $8 \times 4=32$, and the Standard Model representation is 32-dimensional, let us take the tensor product

$$
V=\left(\left(\mathbb{C}^{2} \otimes \mathbb{C}\right) \oplus\left(\mathbb{C} \otimes \mathbb{C}^{2}\right)\right) \otimes\left(\mathbb{C}^{4} \oplus \mathbb{C}^{4 *}\right) .
$$

This becomes a representation of $\mathrm{SU}(2) \times \mathrm{SU}(2) \times \mathrm{SU}(4)$, which we call the PatiSalam representation. To obtain a theory that extends the Standard Model, we also need a way to map $G_{\mathrm{SM}}$ to $\mathrm{SU}(2) \times \mathrm{SU}(2) \times \mathrm{SU}(4)$, such that pulling back $V$ to a representation of $G_{\mathrm{SM}}$ gives the Standard model representation.

How can we map $G_{\mathrm{SM}}$ to $\mathrm{SU}(2) \times \mathrm{SU}(2) \times \mathrm{SU}(4)$ ? There are several possibilities. Our work so far suggests this option:

$$
\begin{aligned}
\mathrm{U}(1) \times \mathrm{SU}(2) \times \mathrm{SU}(3) & \rightarrow \mathrm{SU}(2) \times \mathrm{SU}(2) \times \mathrm{SU}(4), \\
(\alpha, g, h) & \mapsto\left(g, 1,\left(\begin{array}{cc}
\alpha h & 0 \\
0 & \alpha^{-3}
\end{array}\right)\right) .
\end{aligned}
$$

Let us see what this gives. The Pati-Salam representation of $\mathrm{SU}(2) \times \mathrm{SU}(2) \times$ $\mathrm{SU}(4)$ is a direct sum of four irreducibles:

$$
V \cong \mathbb{C}^{2} \otimes \mathbb{C} \otimes \mathbb{C}^{4} \quad \oplus \quad \mathbb{C} \otimes \mathbb{C}^{2} \otimes \mathbb{C}^{4} \quad \oplus \quad \mathbb{C}^{2} \otimes \mathbb{C} \otimes \mathbb{C}^{4 *} \quad \oplus \quad \mathbb{C} \otimes \mathbb{C}^{2} \otimes \mathbb{C}^{4 *}
$$

We hope that the first two will describe left- and right-handed fermions, so let us give them names that suggest this:

$$
\begin{aligned}
& F_{L}=\mathbb{C}^{2} \otimes \mathbb{C} \otimes \mathbb{C}^{4}, \\
& F_{R}=\mathbb{C} \otimes \mathbb{C}^{2} \otimes \mathbb{C}^{4} .
\end{aligned}
$$

The other two are the duals of the first two, since the two-dimensional irrep of $\mathrm{SU}(2)$ is its own dual:

$$
\begin{aligned}
& F_{L}^{*}=\mathbb{C}^{2} \otimes \mathbb{C} \otimes \mathbb{C}^{4 *}, \\
& F_{R}^{*}=\mathbb{C} \otimes \mathbb{C}^{2} \otimes \mathbb{C}^{4 *} .
\end{aligned}
$$


Given our chosen map from $G_{\mathrm{SM}}$ to $\mathrm{SU}(2) \times \mathrm{SU}(2) \times \mathrm{SU}(4)$, we can work out which representations of the $G_{\mathrm{SM}}$ these four spaces give. For example, consider $F_{L}$. We have already seen that under our chosen map,

$$
\mathbb{C}^{4} \cong \mathbb{C}_{\frac{1}{3}} \otimes \mathbb{C}^{3} \oplus \mathbb{C}_{-1} \otimes \mathbb{C}
$$

as representations of $\mathrm{U}(1) \times \mathrm{SU}(3)$, while

$$
\mathbb{C}^{2} \otimes \mathbb{C} \cong \mathbb{C}^{2}
$$

as representations of the left-handed $\mathrm{SU}(2)$. So, as representations of $G_{\mathrm{SM}}$ we have

$$
F_{L} \cong \mathbb{C}_{\frac{1}{3}} \otimes \mathbb{C}^{2} \otimes \mathbb{C}^{3} \oplus \mathbb{C}_{-1} \otimes \mathbb{C}^{2} \otimes \mathbb{C} .
$$

Table 1 shows that these indeed match the left-handed fermions.

If we go ahead and do the other four cases, we see that everything works except for the hypercharges of the right-handed particles and their antiparticles. Here we just show results for the particles:

\begin{tabular}{ccc}
\hline \multicolumn{3}{c}{ The Pati-Salam Model - First Try } \\
\hline Particle & Hypercharge: predicted & Hypercharge: actual \\
\hline$\left(\begin{array}{c}\nu_{L} \\
e_{L}^{-}\end{array}\right.$ & -1 & -1 \\
$u_{L}$ \\
$d_{L}$
\end{tabular}

The problem is that the right-handed particles are getting the same hypercharges as their left-handed brethren. To fix this problem, we need a more clever map from $G_{\mathrm{SM}}$ to $\mathrm{SU}(2) \times \mathrm{SU}(2) \times \mathrm{SU}(4)$. This map must behave differently on the $\mathrm{U}(1)$ factor of $G_{\mathrm{SM}}$, so the hypercharges come out differently, and it must take advantage of the right-handed copy of $\mathrm{SU}(2)$, which acts nontrivially only on the right-handed particles. For example, we can try this map:

$$
\begin{array}{clc}
\mathrm{U}(1) \times \mathrm{SU}(2) \times \mathrm{SU}(3) & \rightarrow & \mathrm{SU}(2) \times \mathrm{SU}(2) \times \mathrm{SU}(4) \\
(\alpha, g, h) & \mapsto\left(g,\left(\begin{array}{cc}
\alpha^{k} & 0 \\
0 & \alpha^{-k}
\end{array}\right),\left(\begin{array}{cc}
\alpha h & 0 \\
0 & \alpha^{-3}
\end{array}\right)\right)
\end{array}
$$

for any integer $k$. This will not affect the above table except for the hypercharges of right-handed particles. It will add $k / 3$ to the hypercharges of the "up" particles in right-handed doublets $\left(\nu_{R}\right.$ and $\left.u_{R}\right)$, and subtract $k / 3$ from the "down" ones $\left(e_{R}^{-}\right.$ and $\left.d_{R}\right)$. So, we obtain these results: 


\begin{tabular}{ccc}
\hline \multicolumn{3}{c}{ The Pati-Salam Model - Second Try } \\
\hline Particle & Hypercharge: predicted & Hypercharge: actual \\
\hline$\left(\begin{array}{c}\nu_{L} \\
e_{L}^{-}\end{array}\right.$ & -1 & -1 \\
$u_{L}$ \\
$d_{L}$
\end{tabular}

Miraculously, all the hypercharges match if we choose $k=3$. So, let us use this map:

$$
\begin{aligned}
\beta: \mathrm{U}(1) \times \mathrm{SU}(2) \times \mathrm{SU}(3) & \rightarrow \quad \mathrm{SU}(2) \times \mathrm{SU}(2) \times \mathrm{SU}(4) \\
(\alpha, g, h) & \mapsto\left(g,\left(\begin{array}{cc}
\alpha^{3} & 0 \\
0 & \alpha^{-3}
\end{array}\right),\left(\begin{array}{cc}
\alpha h & 0 \\
0 & \alpha^{-3}
\end{array}\right)\right) .
\end{aligned}
$$

When we take the Pati-Salam representation of $\mathrm{SU}(2) \times \mathrm{SU}(2) \times \mathrm{SU}(4)$ and pull it back along this map $\beta$, we obtain the Standard Model representation. As in Section 3.1, we use complete reducibility to see this, but we can be more concrete. We saw in Table 4 how we can specify the intertwining map precisely by using a specific basis, which for $\Lambda \mathbb{C}^{5}$ results in the binary code.

Similarly, we can create a kind of "Pati-Salam code" to specify an isomorphism of Hilbert spaces

$$
\ell: F \oplus F^{*} \rightarrow\left(\left(\mathbb{C}^{2} \otimes \mathbb{C}\right) \oplus\left(\mathbb{C} \otimes \mathbb{C}^{2}\right)\right) \otimes\left(\mathbb{C}^{4} \oplus \mathbb{C}^{4 *}\right),
$$

and doing this provides a nice summary of the ideas behind the Pati-Salam model. We take the space $\mathbb{C}^{2} \otimes \mathbb{C}$ to be spanned by $u_{L}$ and $d_{L}$, the left-isospin up and left-isospin down states. Similarly, the space $\mathbb{C} \otimes \mathbb{C}^{2}$ has basis $u_{R}$ and $d_{R}$, called right-isospin up and right-isospin down. Take care not to confuse these with the similarly named quarks. These have no color, and only correspond to isospin.

The color comes from $\mathbb{C}^{4}$ of course, which we already decreed to be spanned by $r, g, b$ and $w$. For antiparticles, we also require anticolors, which we take to be the dual basis $\bar{r}, \bar{g}, \bar{b}$ and $\bar{w}$, spanning $\mathbb{C}^{4 *}$.

It is now easy, with our knowledge of how the Pati-Salam model is to work, to construct this code. Naturally, the left-handed quark doublet corresponds to the left-isospin up and down states, which come in all three colors $c=r, g, b$ :

$$
u_{L}^{c}=u_{L} \otimes c, \quad d_{L}^{c}=d_{L} \otimes c .
$$

The corresponding doublet of left-handed leptons is just the "white" version of this:

$$
\nu_{L}=u_{L} \otimes w, \quad e_{L}^{-}=d_{L} \otimes w .
$$


The right-handed fermions are the same, but with $R$ 's instead of $L$ 's. Thus we get the Pati-Salam code for the fermions:

\begin{tabular}{cc}
\hline$F_{L}$ & $F_{R}$ \\
\hline$\nu_{L}=u_{L} \otimes w$ & $\nu_{R}=u_{R} \otimes w$ \\
$e_{L}^{-}=d_{L} \otimes w$ & $e_{R}^{-}=d_{R} \otimes w$ \\
$u_{L}^{c}=u_{L} \otimes c$ & $u_{R}^{c}=u_{R} \otimes c$ \\
$d_{L}^{c}=d_{L} \otimes c$ & $d_{R}^{c}=d_{R} \otimes c$ \\
\hline
\end{tabular}

The result is very similar for the antifermions in $F_{L}^{*}$ and $F_{R}^{*}$, but watch out: taking antiparticles swaps up and down, and also swaps left and right, so the particles in $F_{L}^{*}$ are right-handed, despite the subscript $L$, while those in $F_{R}^{*}$ are left-handed. This is because it is the right-handed antiparticles that feel the weak force, which in terms of representation theory means they are nontrivial under the left $\mathrm{SU}(2)$. So, the Pati-Salam code for the antifermions is this:

\begin{tabular}{cc}
\hline$F_{L}^{*}$ & $F_{R}^{*}$ \\
\hline$e_{R}^{+}=u_{L} \otimes \bar{w}$ & $e_{L}^{+}=u_{R} \otimes \bar{w}$ \\
$\bar{\nu}_{R}=d_{L} \otimes \bar{w}$ & $\bar{\nu}_{L}=d_{R} \otimes \bar{w}$ \\
$\bar{d}_{R}^{\bar{c}}=u_{L} \otimes \bar{c}$ & $\bar{d}_{L}^{c}=u_{R} \otimes \bar{c}$ \\
$\bar{u}_{R}^{\bar{c}}=d_{L} \otimes \bar{c}$ & $\bar{u}_{L}^{c}=d_{R} \otimes \bar{c}$ \\
\hline
\end{tabular}

Putting these together we get the full Pati-Salam code:

TABLE 5. Pati-Salam code for first-generation fermions, where $c=$ $r, g, b$ and $\bar{c}=\bar{r}, \bar{b}, \bar{g}$.

\begin{tabular}{cccc}
\hline \multicolumn{5}{c}{ The Pati-Salam Code } \\
\hline$F_{L}$ & $F_{R}$ & $F_{L}^{*}$ & $F_{R}^{*}$ \\
\hline$\nu_{L}=u_{L} \otimes w$ & $\nu_{R}=u_{R} \otimes w$ & $e_{R}^{+}=u_{L} \otimes \bar{w}$ & $e_{L}^{+}=u_{R} \otimes \bar{w}$ \\
$e_{L}^{-}=d_{L} \otimes w$ & $e_{R}^{-}=d_{R} \otimes w$ & $\bar{\nu}_{R}=d_{L} \otimes \bar{w}$ & $\bar{\nu}_{L}=d_{R} \otimes \bar{w}$ \\
$u_{L}^{c}=u_{L} \otimes c$ & $u_{R}^{c}=u_{R} \otimes c$ & $\bar{d}_{R}^{\bar{c}}=u_{L} \otimes \bar{c}$ & $\bar{d}_{L}^{c}=u_{R} \otimes \bar{c}$ \\
$d_{L}^{c}=d_{L} \otimes c$ & $d_{R}^{c}=d_{R} \otimes c$ & $\bar{u}_{R}^{\bar{c}}=d_{L} \otimes \bar{c}$ & $\bar{u}_{L}^{\bar{c}}=d_{R} \otimes \bar{c}$ \\
\hline
\end{tabular}

This table defines an isomorphism of Hilbert spaces

$$
\ell: F \oplus F^{*} \rightarrow\left(\left(\mathbb{C}^{2} \otimes \mathbb{C}\right) \oplus\left(\mathbb{C} \otimes \mathbb{C}^{2}\right)\right) \otimes\left(\mathbb{C}^{4} \oplus \mathbb{C}^{4 *}\right),
$$

so where it says, for example, $\nu_{L}=u_{L} \otimes w$, that is just short for $\ell\left(\nu_{L}\right)=u_{L} \otimes w$. This map $\ell$ is also an isomorphism between representations of $G_{\mathrm{SM}}$. It tells us how these representations are the "same", just as the map $h$ did for $F \oplus F^{*}$ and $\Lambda \mathbb{C}^{5}$ at the end of Section 3.1 . 
As with SU(5) and Spin(10), we can summarize all the results of this section in a commutative square:

Theorem 3. The following square commutes:

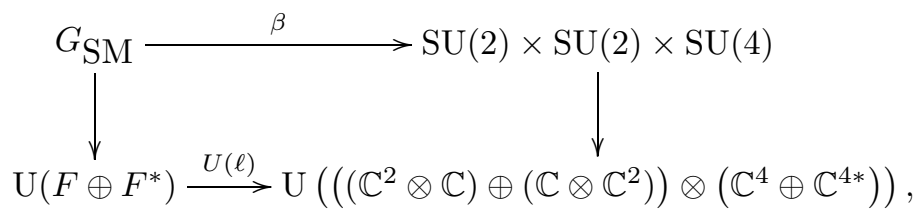

where the left vertical arrow is the Standard Model representation and the right one is the Pati-Salam representation.

The Pati-Salam representation and especially the homomorphism $\beta$ look less natural than the representation of $\mathrm{SU}(5)$ on $\Lambda \mathbb{C}^{5}$ and the homomorphism $\phi: G_{\mathrm{SM}} \rightarrow$ $\mathrm{SU}(5)$. But appearances can be deceiving: in the next section we shall see a more elegant way to describe them.

3.4. The route to $\mathbf{S p i n}(\mathbf{1 0})$ via Pati-Salam. In the last section, we showed how the Pati-Salam model answers two questions about the Standard Model:

Why are quarks and leptons so similar? Why are left and right so different?

We were able to describe leptons as a fourth color of quark, "white", and treat right-handed and left-handed particles on a more equal footing. Neither of these ideas worked on its own, but together, they made a full-fledged extension of the Standard Model, much like SU(5) and Spin(10), but based on seemingly different principles.

Yet thinking of leptons as "white" should be strangely familiar, not just from the Pati-Salam perspective, but from the binary code that underlies both the SU(5) and the Spin(10) theories. There, leptons were indeed white: they all have color $r \wedge g \wedge b \in \Lambda \mathbb{C}^{5}$.

Alas, while $\mathrm{SU}(5)$ hints that leptons might be a fourth color, it does not deliver on this. The quark colors

$$
r, g, b \in \Lambda^{1} \mathbb{C}^{5}
$$

lie in a different irrep of $\mathrm{SU}(5)$ than does $r \wedge g \wedge b \in \Lambda^{3} \mathbb{C}^{5}$. So, leptons in the $\mathrm{SU}(5)$ theory are white, but unlike the Pati-Salam model, this theory does not unify leptons with quarks.

Yet $\mathrm{SU}(5)$ theory is not the only game in town when it comes to the binary code. We also have $\operatorname{Spin}(10)$, which acts on the same vector space as $\mathrm{SU}(5)$. As a representation of $\operatorname{Spin}(10), \Lambda \mathbb{C}^{5}$ breaks up into just two irreps: the even grades, $\Lambda^{\mathrm{ev}} \mathbb{C}^{5}$, which contain the left-handed particles and antiparticles:

$$
\Lambda^{\mathrm{ev}} \mathbb{C}^{5} \cong\left\langle\bar{\nu}_{L}\right\rangle \oplus\left\langle e_{L}^{+}\right\rangle \oplus\left\langle\begin{array}{c}
u_{L} \\
d_{L}
\end{array}\right\rangle \oplus\left\langle\bar{u}_{L}\right\rangle \oplus\left\langle\begin{array}{c}
\nu_{L} \\
e_{L}^{-}
\end{array}\right\rangle \oplus\left\langle\bar{d}_{L}\right\rangle
$$

and the odd grades $\Lambda^{\text {odd }} \mathbb{C}^{5}$, which contain the right-handed particles and antiparticles:

$$
\Lambda^{\text {odd }} \mathbb{C}^{5} \cong\left\langle\nu_{R}\right\rangle \oplus\left\langle e_{R}^{-}\right\rangle \oplus\left\langle\begin{array}{c}
\bar{d}_{R} \\
\bar{u}_{R}
\end{array}\right\rangle \oplus\left\langle u_{R}\right\rangle \oplus\left\langle\begin{array}{c}
e_{R}^{+} \\
\bar{\nu}_{R}
\end{array}\right\rangle \oplus\left\langle d_{R}\right\rangle .
$$

Unlike SU(5), the Spin(10) GUT really does unify $r \wedge g \wedge b$ with the colors $r, g$ and $b$, because they both live in the irrep $\Lambda^{\text {odd }} \mathbb{C}^{5}$. 
In short, it seems that the Spin(10) GUT, which we built as an extension of the SU(5) GUT, somehow managed to pick up this feature of the Pati-Salam model. How does $\mathrm{Spin}(10)$ relate to Pati-Salam's gauge group $\mathrm{SU}(2) \times \mathrm{SU}(2) \times \mathrm{SU}(4)$, exactly? In general, we only know there is a map $\mathrm{SU}(n) \rightarrow \operatorname{Spin}(2 n)$, but in low dimensions, there is much more, because some groups coincide:

$$
\begin{aligned}
& \operatorname{Spin}(3) \cong \mathrm{SU}(2), \\
& \operatorname{Spin}(4) \cong \mathrm{SU}(2) \times \mathrm{SU}(2), \\
& \mathrm{Spin}(5) \cong \mathrm{Sp}(2), \\
& \operatorname{Spin}(6) \cong \mathrm{SU}(4) .
\end{aligned}
$$

What really stands out is this:

$$
\mathrm{SU}(2) \times \mathrm{SU}(2) \times \mathrm{SU}(4) \cong \mathrm{Spin}(4) \times \operatorname{Spin}(6) .
$$

This brings out an obvious relationship between the Pati-Salam model and the $\operatorname{Spin}(10)$ theory, because the inclusion $\mathrm{SO}(4) \times \mathrm{SO}(6) \hookrightarrow \mathrm{SO}(10)$ lifts to the universal covers, so we get a homomorphism

$$
\eta: \operatorname{Spin}(4) \times \operatorname{Spin}(6) \rightarrow \operatorname{Spin}(10) .
$$

A word of caution is needed here. While $\eta$ is the lift of an inclusion, it is not an inclusion itself: it is two-to-one. This is because the universal cover $\operatorname{Spin}(4) \times \operatorname{Spin}(6)$ of $\mathrm{SO}(4) \times \mathrm{SO}(6)$ is a four-fold cover, being a double cover on each factor.

So we can try to extend the symmetries $\mathrm{SU}(2) \times \mathrm{SU}(2) \times \mathrm{SU}(4)$ to $\mathrm{Spin}(10)$, though this can only work if the kernel of $\eta$ acts trivially on the Pati-Salam representation. What is this representation like? There is an obvious representation of $\operatorname{Spin}(4) \times \operatorname{Spin}(6)$ that extends to a $\operatorname{Spin}(10)$ representation. Both $\operatorname{Spin}(4)$ and Spin(6) have Dirac spinor representations, so their product $\operatorname{Spin}(4) \times \operatorname{Spin}(6)$ has a representation on $\Lambda \mathbb{C}^{2} \otimes \Lambda \mathbb{C}^{3}$. In fact, the obvious map

$$
g: \Lambda \mathbb{C}^{2} \otimes \Lambda \mathbb{C}^{3} \rightarrow \Lambda \mathbb{C}^{5}
$$

given by

$$
v \otimes w \mapsto v \wedge w
$$

is an isomorphism compatible with the actions of $\operatorname{Spin}(4) \times \operatorname{Spin}(6)$ on these two spaces. More concisely, this square:

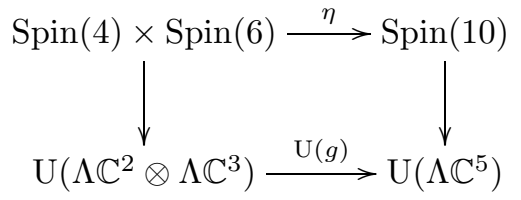

commutes.

We will prove this in a moment. First though, we must check that this representation of $\operatorname{Spin}(4) \times \operatorname{Spin}(6)$ is secretly just another name for the Pati-Salam representation of $\mathrm{SU}(2) \times \mathrm{SU}(2) \times \mathrm{SU}(4)$ on the space we discussed in Section 3.3 .

$$
\left(\left(\mathbb{C}^{2} \otimes \mathbb{C}\right) \oplus\left(\mathbb{C} \otimes \mathbb{C}^{2}\right)\right) \otimes\left(\mathbb{C}^{4} \oplus \mathbb{C}^{4 *}\right) .
$$

Checking this involves choosing an isomorphism between $\mathrm{SU}(2) \times \mathrm{SU}(2) \times \mathrm{SU}(4)$ and $\operatorname{Spin}(4) \times \operatorname{Spin}(6)$. Luckily, we can choose one that works: 
Theorem 4. There exists an isomorphism of Lie groups

$$
\alpha: \mathrm{SU}(2) \times \mathrm{SU}(2) \times \mathrm{SU}(4) \rightarrow \operatorname{Spin}(4) \times \operatorname{Spin}(6)
$$

and a unitary operator

$$
k:\left(\left(\mathbb{C}^{2} \otimes \mathbb{C}\right) \oplus\left(\mathbb{C} \otimes \mathbb{C}^{2}\right)\right) \otimes\left(\mathbb{C}^{4} \oplus \mathbb{C}^{4 *}\right) \rightarrow \Lambda \mathbb{C}^{2} \otimes \Lambda \mathbb{C}^{3}
$$

that make this square commute:

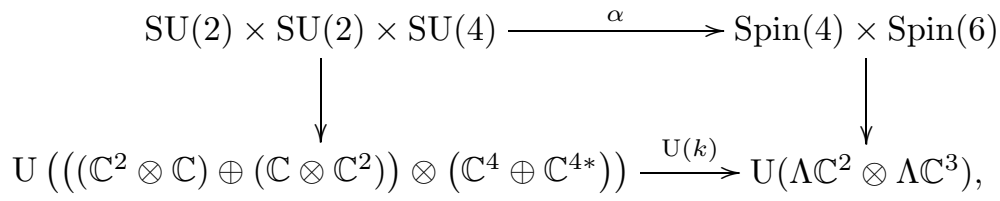

where the left vertical arrow is the Pati-Salam representation and the right one is a tensor product of Dirac spinor representations.

Proof. We can prove this in pieces, by separately finding a unitary operator

$$
\left(\mathbb{C}^{2} \otimes \mathbb{C}\right) \oplus\left(\mathbb{C} \otimes \mathbb{C}^{2}\right) \cong \Lambda \mathbb{C}^{2}
$$

that makes this square commute:

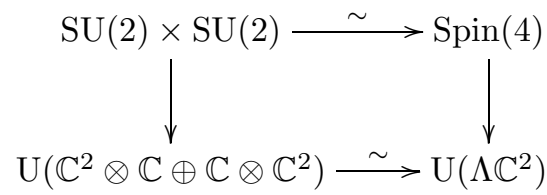

and a unitary operator

$$
\mathbb{C}^{4} \oplus \mathbb{C}^{4 *} \cong \Lambda \mathbb{C}^{3}
$$

that make this square:

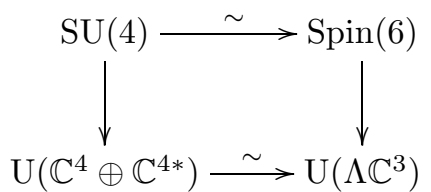

commute.

First, the Spin(6) piece. It suffices to show that the Dirac spinor representation of $\operatorname{Spin}(6) \cong \mathrm{SU}(4)$ on $\Lambda \mathbb{C}^{3}$ is isomorphic to $\mathbb{C}^{4} \oplus \mathbb{C}^{4 *}$ as a representation of $\mathrm{SU}(4)$. We start with the action of $\operatorname{Spin}(6)$ on $\Lambda \mathbb{C}^{3}$. This breaks up into irreps:

$$
\Lambda \mathbb{C}^{3} \cong \Lambda^{\mathrm{ev}} \mathbb{C}^{3} \oplus \Lambda^{\text {odd }} \mathbb{C}^{3}
$$

called the left-handed and right-handed Weyl spinors, and these are dual to each other because $6=2 \bmod 4$, by a theorem that can be found in Adams' lectures [1. Call these representations

$$
\rho_{\text {ev }}: \operatorname{Spin}(6) \rightarrow \mathrm{U}\left(\Lambda^{\mathrm{ev}} \mathbb{C}^{3}\right), \quad \rho_{\text {odd }}: \operatorname{Spin}(6) \rightarrow \mathrm{U}\left(\Lambda^{\text {odd }} \mathbb{C}^{3}\right) .
$$

Since these representations are dual, it suffices just to consider one of them, say $\rho_{\text {odd }}$.

Passing to Lie algebras, we have a homomorphism

$$
d \rho_{\text {odd }}: \mathfrak{s o}(6) \rightarrow \mathfrak{u}\left(\Lambda^{\text {odd }} \mathbb{C}^{3}\right) \cong \mathfrak{u}(4) \cong \mathfrak{u}(1) \oplus \mathfrak{s u}(4) .
$$


Homomorphic images of semisimple Lie algebras are semisimple, so the image of $\mathfrak{s o}(6)$ must lie entirely in $\mathfrak{s u}(4)$. In fact $\mathfrak{s o}(6)$ is simple, so this nontrivial map must be an injection

$$
d \rho_{\text {odd }}: \mathfrak{s o}(6) \rightarrow \mathfrak{s u}(4),
$$

and because the dimension is 15 on both sides, this map is also onto. Thus $d \rho_{\text {odd }}$ is an isomorphism of Lie algebras, so $\rho_{\text {odd }}$ is an isomorphism of the simply connected Lie groups $\operatorname{Spin}(6)$ and $\mathrm{SU}(4)$ :

$$
\rho_{\text {odd }}: \operatorname{Spin}(6) \rightarrow \mathrm{SU}\left(\Lambda^{\text {odd }} \mathbb{C}^{3}\right) \cong \mathrm{SU}(4) .
$$

Furthermore, under this isomorphism,

$$
\Lambda^{\text {odd }} \mathbb{C}^{3} \cong \mathbb{C}^{4}
$$

as a representation of $\mathrm{SU}\left(\Lambda^{\text {odd }} \mathbb{C}^{3}\right) \cong \mathrm{SU}(4)$. Taking duals, we obtain an isomorphism

$$
\Lambda^{\mathrm{ev}} \mathbb{C}^{3} \cong \mathbb{C}^{4 *}
$$

Putting these together, we get an isomorphism $\mathbb{C}^{4} \oplus \mathbb{C}^{4 *} \cong \Lambda \mathbb{C}^{3}$ that makes this square commute:

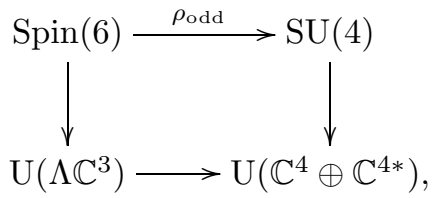

which completes the proof for Spin(6).

Next we consider the Spin(4) piece. It suffices to show that the spinor representation $\Lambda \mathbb{C}^{2}$ of $\operatorname{Spin}(4) \cong \mathrm{SU}(2) \times \mathrm{SU}(2)$ is isomorphic to $\mathbb{C}^{2} \otimes \mathbb{C} \oplus \mathbb{C} \otimes \mathbb{C}^{2}$ as a representation of $\mathrm{SU}(2) \times \mathrm{SU}(2)$. We start with the action of $\operatorname{Spin}(4)$ on $\Lambda \mathbb{C}^{2}$. This again breaks up into irreps:

$$
\Lambda \mathbb{C}^{2} \cong \Lambda^{\text {odd }} \mathbb{C}^{2} \oplus \Lambda^{\mathrm{ev}} \mathbb{C}^{2}
$$

Again we call these representations

$$
\rho_{\text {ev }}: \operatorname{Spin}(4) \rightarrow \mathrm{U}\left(\Lambda^{\mathrm{ev}} \mathbb{C}^{2}\right), \quad \rho_{\text {odd }}: \operatorname{Spin}(4) \rightarrow \mathrm{U}\left(\Lambda^{\text {odd }} \mathbb{C}^{2}\right) .
$$

First consider $\rho_{\mathrm{ev}}$. Passing to Lie algebras, this gives a homomorphism

$$
d \rho_{\mathrm{ev}}: \mathfrak{s o}(4) \rightarrow \mathfrak{u}\left(\Lambda^{\mathrm{ev}} \mathbb{C}^{2}\right) \cong \mathfrak{u}(2) \cong \mathfrak{u}(1) \oplus \mathfrak{s} \mathfrak{u}(2) .
$$

Homomorphic images of semisimple Lie algebras are semisimple, so the image of $\mathfrak{s o}(4)$ must lie entirely in $\mathfrak{s u}(2)$. Similarly, $d \rho_{\text {odd }}$ also takes $\mathfrak{s o}(4)$ to $\mathfrak{s u}(2)$ :

$$
d \rho_{\text {odd }}: \mathfrak{s o}(4) \rightarrow \mathfrak{s u}(2)
$$

and we can combine these maps to get

$$
d \rho_{\text {odd }} \oplus d \rho_{\text {ev }}: \mathfrak{s o}(4) \rightarrow \mathfrak{s u}(2) \oplus \mathfrak{s u}(2),
$$

which is just the derivative of $\operatorname{Spin}(4)$ 's representation on $\Lambda \mathbb{C}^{2}$. Since this representation is faithful, the map $d \rho_{\text {odd }} \oplus d \rho_{\mathrm{ev}}$ of Lie algebras is injective. But the dimensions of $\mathfrak{s o}(4)$ and $\mathfrak{s u}(2) \oplus \mathfrak{s u}(2)$ agree, so $d \rho_{\text {odd }} \oplus d \rho_{\text {ev }}$ is also onto. Thus it is an isomorphism of Lie algebras. This implies that $\rho_{\text {odd }} \oplus \rho_{\mathrm{ev}}$ is an isomorphism of the simply connected Lie groups $\operatorname{Spin}(4)$ and $\mathrm{SU}(2) \times \mathrm{SU}(2)$,

$$
\rho_{\text {odd }} \oplus \rho_{\text {ev }}: \operatorname{Spin}(4) \rightarrow \mathrm{SU}\left(\Lambda^{\text {odd }} \mathbb{C}^{2}\right) \times \mathrm{SU}\left(\Lambda^{\mathrm{ev}} \mathbb{C}^{2}\right) \cong \mathrm{SU}(2) \times \mathrm{SU}(2),
$$


under which $\mathrm{SU}(2) \times \mathrm{SU}(2)$ acts on $\Lambda^{\text {odd }} \mathbb{C}^{2} \oplus \Lambda^{\mathrm{ev}} \mathbb{C}^{2}$. The left factor of $\mathrm{SU}(2)$ acts irreducibly on $\Lambda^{\text {odd }} \mathbb{C}^{2}$, which the second factor is trivial on. Thus $\Lambda^{\text {odd }} \mathbb{C}^{2} \cong \mathbb{C}^{2} \otimes \mathbb{C}$ as a representation of $\mathrm{SU}(2) \times \mathrm{SU}(2)$. Similarly, $\Lambda^{\mathrm{ev}} \mathbb{C}^{2} \cong \mathbb{C} \otimes \mathbb{C}^{2}$ as a representation of this group. Putting these together, we get an isomorphism $\mathbb{C}^{2} \otimes \mathbb{C} \oplus \mathbb{C} \otimes \mathbb{C}^{2} \cong \Lambda \mathbb{C}^{2}$ that makes this square commute:

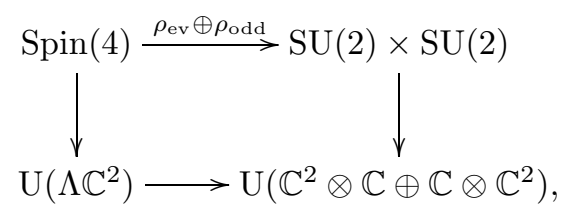

which completes the proof for $\operatorname{Spin}(4)$.

In the proof of the preceding theorem, we merely showed that there exists an isomorphism

$$
k:\left(\left(\mathbb{C}^{2} \otimes \mathbb{C}\right) \oplus\left(\mathbb{C} \otimes \mathbb{C}^{2}\right)\right) \otimes\left(\mathbb{C}^{4} \oplus \mathbb{C}^{4 *}\right) \rightarrow \Lambda \mathbb{C}^{2} \otimes \Lambda \mathbb{C}^{3}
$$

making the square commute. We did not say exactly what $k$ was. In the proof, we built it after quietly choosing three unitary operators, giving these isomorphisms:

$$
\mathbb{C}^{2} \otimes \mathbb{C} \cong \Lambda^{\text {odd }} \mathbb{C}^{2}, \quad \mathbb{C} \otimes \mathbb{C}^{2} \cong \Lambda^{\mathrm{ev}} \mathbb{C}^{2}, \quad \mathbb{C}^{4} \cong \Lambda^{\text {odd }} \mathbb{C}^{3} .
$$

Since the remaining map $\Lambda^{\mathrm{ev}} \mathbb{C}^{3} \cong \mathbb{C}^{4 *}$ is determined by duality, these three operators determine $k$, and they also determine the Lie group isomorphism $\alpha$ via the construction in our proof.

There is, however, a specific choice for these unitary operators that we prefer, because this choice makes the particles in the Pati-Salam representation $\Lambda \mathbb{C}^{2} \otimes \Lambda \mathbb{C}^{3}$ look almost exactly like those in the $\mathrm{SU}(5)$ representation $\Lambda \mathbb{C}^{5}$.

First, since $\mathbb{C}^{2}=\Lambda^{1} \mathbb{C}^{2}=\Lambda^{\text {odd }} \mathbb{C}^{2}$ is spanned by $u$ and $d$, the (left-handed) isospin states of the Standard Model, we really ought to identify the left-isospin states $u_{L}$ and $d_{L}$ of the Pati-Salam model with these. So, we should use this unitary operator:

$$
\begin{aligned}
\mathbb{C}^{2} \otimes \mathbb{C} & \stackrel{\sim}{\longrightarrow} \Lambda^{\text {odd }} \mathbb{C}^{2} \\
u_{L} & \mapsto u \\
d_{L} & \mapsto d .
\end{aligned}
$$

Next, we should use this unitary operator for right-isospin states:

$$
\begin{aligned}
\mathbb{C} \otimes \mathbb{C}^{2} & \stackrel{\sim}{\longrightarrow} \Lambda^{\mathrm{ev}} \mathbb{C}^{2} \\
u_{R} & \mapsto u \wedge d \\
d_{R} & \mapsto 1 .
\end{aligned}
$$

Why? Because the right-isospin up particle is the right-handed neutrino $\nu_{R}$, which corresponds to $u \wedge d \wedge r \wedge g \wedge b$ in the $\mathrm{SU}(5)$ theory, but $u_{R} \otimes w$ in the Pati-Salam model. This suggests that $u \wedge d$ and $u_{R}$ should be identified.

Finally, because $\mathbb{C}^{3}$ is spanned by the colors $r, g$ and $b$, while $\mathbb{C}^{4}$ is spanned by the colors $r, g, b$ and $w$, we really ought to use this unitary operator:

$$
\begin{array}{rll}
\mathbb{C}^{4} & \stackrel{\longrightarrow}{ } \Lambda^{\text {odd }} \mathbb{C}^{3} \\
r & \mapsto & r \\
g & \mapsto & g \\
b & \mapsto & b \\
w & \mapsto & r \wedge g \wedge b .
\end{array}
$$


Dualizing this, we get the unitary operator

$$
\begin{array}{rll}
\mathbb{C}^{4 *} & \stackrel{\sim}{\longrightarrow} \Lambda^{\mathrm{ev}} \mathbb{C}^{3} \\
\bar{r} & \mapsto & g \wedge b \\
\bar{g} & \mapsto & b \wedge r \\
\bar{b} & \mapsto & r \wedge g \\
\bar{w} & \mapsto & 1 .
\end{array}
$$

These choices determine the unitary operator

$$
k:\left(\left(\mathbb{C}^{2} \otimes \mathbb{C}\right) \oplus\left(\mathbb{C} \otimes \mathbb{C}^{2}\right)\right) \otimes\left(\mathbb{C}^{4} \oplus \mathbb{C}^{4 *}\right) \rightarrow \Lambda \mathbb{C}^{2} \otimes \Lambda \mathbb{C}^{3} .
$$

With this specific choice of $k$, we can combine the commutative squares built in Theorems 3 and 4 .

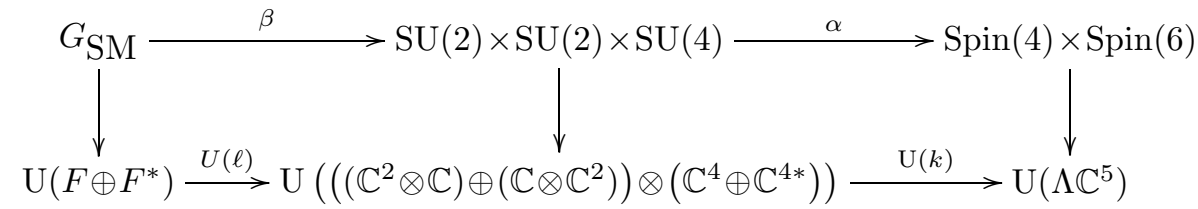

to obtain the following result:

Theorem 5. Taking $\theta=\alpha \beta$ and $h=k \ell$, the following square commutes:

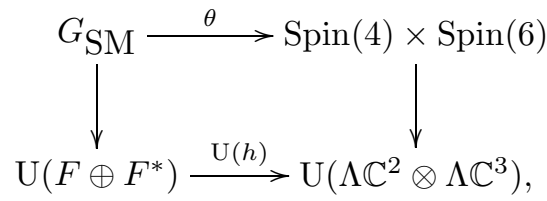

where the left vertical arrow is the Standard Model representation and the right one is a tensor product of Dirac spinor representations.

The map $h$, which tells us how to identify $F \oplus F^{*}$ and $\Lambda \mathbb{C}^{2} \otimes \Lambda \mathbb{C}^{3}$, is given by applying $k$ to the "Pati-Salam code" in Table 5. This gives a binary code for the Pati-Salam model:

TABLE 6. Pati-Salam binary code for first-generation fermions, where $c=r, g, b$ and $\bar{c}=g b, b r, r g$.

\begin{tabular}{cccc}
\hline \multicolumn{4}{c}{ The Binary Code for Pati-Salam } \\
\hline$\Lambda^{\text {odd }} \mathbb{C}^{2} \otimes \Lambda^{\text {odd }} \mathbb{C}^{3}$ & $\Lambda^{\text {ev }} \mathbb{C}^{2} \otimes \Lambda^{\text {odd }} \mathbb{C}^{3}$ & $\Lambda^{\text {odd }} \mathbb{C}^{2} \otimes \Lambda^{\text {ev }} \mathbb{C}^{3}$ & $\Lambda^{\text {ev }} \mathbb{C}^{2} \otimes \Lambda^{\text {ev }} \mathbb{C}^{3}$ \\
\hline$\nu_{L}=u \otimes r g b$ & $\nu_{R}=u d \otimes r g b$ & $e_{R}^{+}=u \otimes 1$ & $e_{L}^{+}=u d \otimes 1$ \\
$e_{L}^{-}=d \otimes r g b$ & $e_{R}^{-}=1 \otimes r g b$ & $\bar{\nu}_{R}=d \otimes 1$ & $\bar{\nu}_{L}=1 \otimes 1$ \\
$u_{L}^{c}=u \otimes c$ & $u_{R}^{c}=u d \otimes c$ & $\bar{d}_{R}^{\bar{c}}=u \otimes \bar{c}$ & $\bar{d}_{L}^{\bar{c}}=u d \otimes \bar{c}$ \\
$d_{L}^{c}=d \otimes c$ & $d_{R}^{c}=1 \otimes c$ & $\bar{u}_{R}^{\bar{c}}=d \otimes \bar{c}$ & $\bar{u}_{L}^{\bar{c}}=1 \otimes \bar{c}$ \\
\hline
\end{tabular}

We have omitted wedge product symbols to save space. Note that if we apply the obvious isomorphism

$$
g: \Lambda \mathbb{C}^{2} \otimes \Lambda \mathbb{C}^{3} \rightarrow \Lambda \mathbb{C}^{5}
$$

given by

$$
v \otimes w \mapsto v \wedge w
$$


then the above table does more than merely resemble Table 4 , which gives the binary code for the SU(5) theory. The two tables become identical!

This fact is quite intriguing. We will explore its meaning in the next section. But first, let us start by relating the Pati-Salam model to the Spin(10) theory:

Theorem 6. The following square commutes:

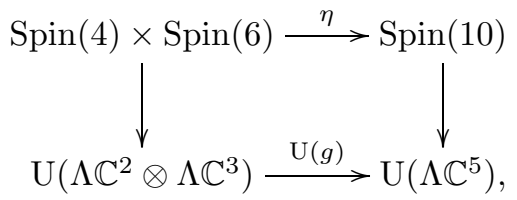

where the right vertical arrow is the Dirac spinor representation, the left one is the tensor product of Dirac spinor representations, and

$$
\eta: \operatorname{Spin}(4) \times \operatorname{Spin}(6) \rightarrow \operatorname{Spin}(10)
$$

is the homomorphism lifting the inclusion of $\mathrm{SO}(4) \times \mathrm{SO}(6)$ in $\mathrm{SO}(10)$.

Proof. At the Lie algebra level, we have the inclusion

$$
\mathfrak{s o}(4) \oplus \mathfrak{s o}(6) \hookrightarrow \mathfrak{s o}(10)
$$

by block diagonals, which is also just the differential of the inclusion $\mathrm{SO}(4) \times$ $\mathrm{SO}(6) \hookrightarrow \mathrm{SO}(10)$ at the Lie group level. Given how the spinor representations are defined in terms of creation and annihilation operators, it is easy to see that

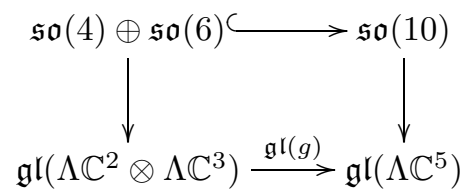

commutes, because $g$ is an intertwining operator between representations of $\mathfrak{s o}(4) \oplus$ $\mathfrak{s o}(6)$, that is, because the $\mathfrak{s o}(4)$ part only acts on $\Lambda \mathbb{C}^{2}$, while the $\mathfrak{s o}(6)$ part only acts on $\Lambda \mathbb{C}^{3}$.

But these Lie algebras act by skew-adjoint operators, so really

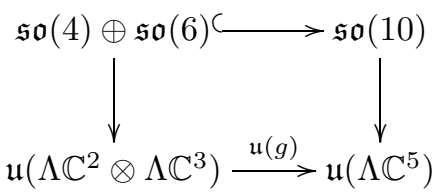

commutes. Since the $\mathfrak{s o}(n)$ 's and their direct sums are semisimple, so are their images. Therefore, their images live in the semisimple part of the unitary Lie algebras, which is just another way of saying the special unitary Lie algebras. We get that

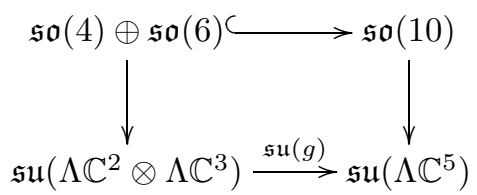


commutes, and this gives a commutative square in the world of simply connected Lie groups:

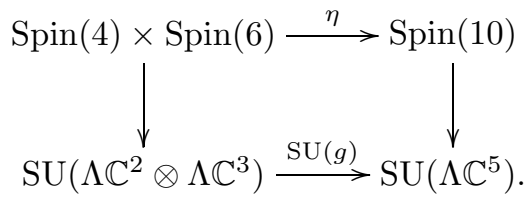

This completes the proof.

This result shows us how to reach the Spin(10) theory, not through the SU(5) theory, but through the Pati-Salam model. For physics texts that treat this issue, see for example Zee [40] and Ross [31].

3.5. The question of compatibility. We now have two routes to the $\operatorname{Spin}(10)$ theory. In Section 3.2 we saw how to reach it via the $\mathrm{SU}(5)$ theory:

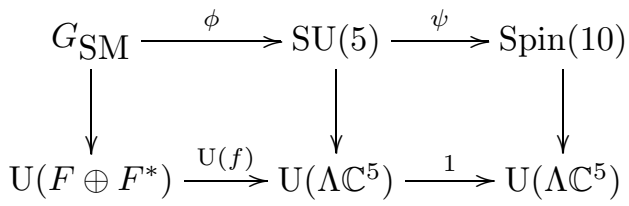

More $\widetilde{\text { Unification }}$

Our work in that section and in Section 3.1 showed that this diagram commutes, which is a way of saying that the Spin(10) theory extends the Standard Model.

In Section 3.4 we saw another route to the $\operatorname{Spin}(10)$ theory, which goes through $\operatorname{Spin}(4) \times \operatorname{Spin}(6):$

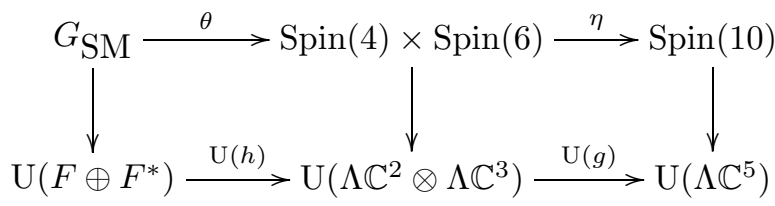

More Unification

Our work in that section and Section 3.3 showed that this diagram commutes as well. So, we have another way to extend the Standard Model and get the Spin(10) theory.

Drawing these two routes to $\operatorname{Spin}(10)$ together gives us a cube:

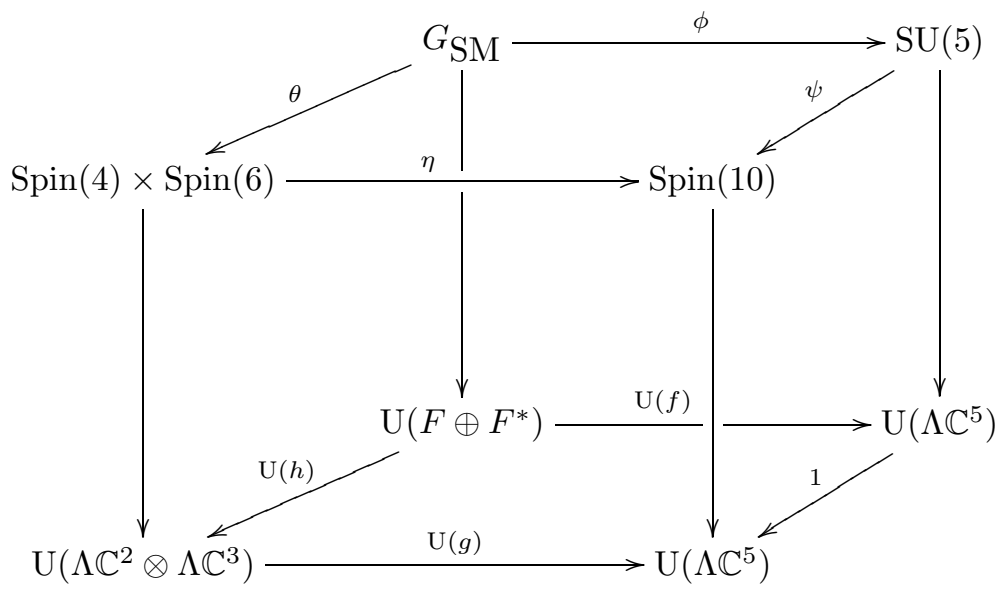


Are these two routes to Spin(10) theory the same? That is, does the cube commute?

Theorem 7. The cube commutes.

Proof. We have already seen in Sections 3.13 .4 that the vertical faces commute. So, we are left with two questions involving the horizontal faces. First: does the top face of the cube

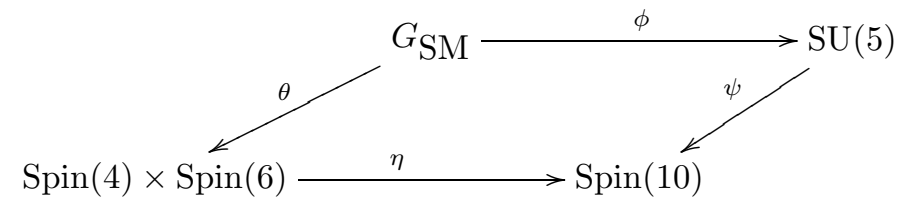

commute? In other words: does a symmetry in $G_{\mathrm{SM}}$ go to the same place in Spin(10) no matter how we take it there? Second: does the bottom face of the cube commute? In other words: does this triangle:

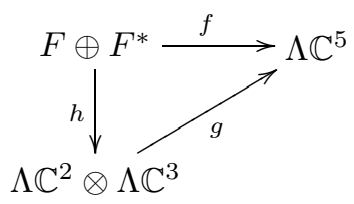

commute?

In fact they both do, and we can use our affirmative answer to the second question to settle the first. As we remarked in Section 3.4 applying the map $g$ to the PatiSalam binary code given in Table 6, we get the $\mathrm{SU}(5)$ binary code given in Table 4 . Thus, the linear maps $f$ and $g h$ agree on a basis, so this triangle commutes:

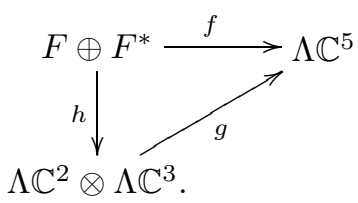

This in turn implies that the bottom face of the cube commutes, from which we see that the two maps from $G_{\mathrm{SM}}$ to $\mathrm{U}\left(\Lambda \mathbb{C}^{5}\right)$ going around the bottom face are equal:

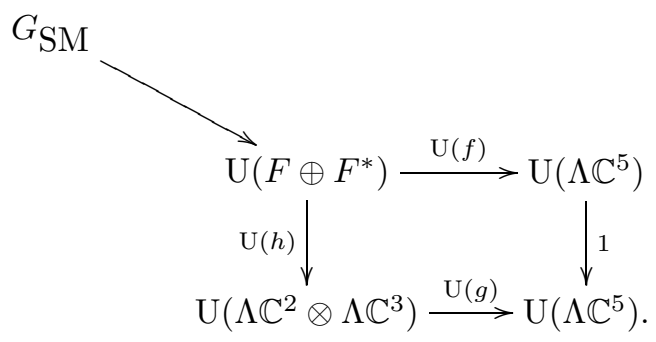

The work of Section 3.1 through Section 3.4 showed that the vertical faces of the cube commute. We can thus conclude from diagrammatic reasoning that the two 
maps from $G_{\mathrm{SM}}$ to $\mathrm{U}\left(\Lambda \mathbb{C}^{5}\right)$ going around the top face are equal:

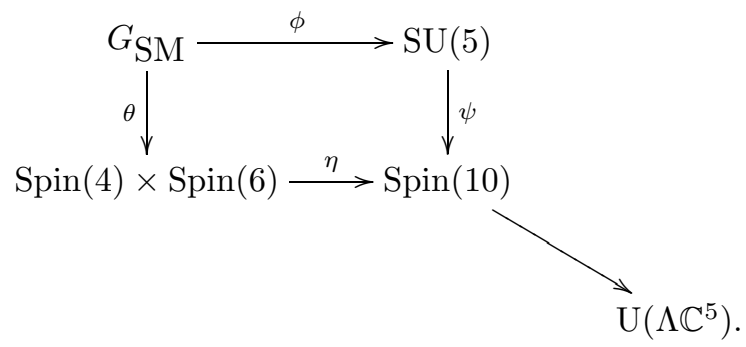

Since the Dirac spinor representation is faithful, the map $\operatorname{Spin}(10) \rightarrow \mathrm{U}\left(\Lambda \mathbb{C}^{5}\right)$ is injective. This means we can drop it from the above diagram, and the remaining square commutes. But this is exactly the top face of the cube. So, the proof is done.

\section{Conclusion}

We have studied three different grand unified theories: the $\mathrm{SU}(5), \operatorname{Spin}(4) \times$ $\operatorname{Spin}(6)$ and $\operatorname{Spin}(10)$ theories. The $\mathrm{SU}(5)$ and $\operatorname{Spin}(4) \times \operatorname{Spin}(6)$ theories were based on different visions about how to extend the Standard Model. However, we saw that both of these theories can be extended to the $\operatorname{Spin}(10)$ theory, which therefore unites these visions.

The $\mathrm{SU}(5)$ theory is all about treating isospin and color on an equal footing: it combines the two isospins of $\mathbb{C}^{2}$ with the three colors of $\mathbb{C}^{3}$, and posits an SU(5) symmetry acting on the resulting $\mathbb{C}^{5}$. The particles and antiparticles in a single generation of fermions are described by vectors in $\Lambda \mathbb{C}^{5}$. So, we can describe each of these particles and antiparticles by a binary code indicating the presence or absence of up, down, red, green and blue.

In doing so, the $\mathrm{SU}(5)$ theory introduces unexpected relationships between matter and antimatter. The irreducible representations of $\mathrm{SU}(5)$

$$
\Lambda^{0} \mathbb{C}^{5} \oplus \Lambda^{1} \mathbb{C}^{5} \oplus \Lambda^{2} \mathbb{C}^{5} \oplus \Lambda^{3} \mathbb{C}^{5} \oplus \Lambda^{4} \mathbb{C}^{5} \oplus \Lambda^{5} \mathbb{C}^{5}
$$

unify some particles we normally consider to be "matter" with some we normally consider "antimatter", as in

$$
\Lambda^{1} \mathbb{C}^{5} \cong\left\langle\begin{array}{c}
e_{R}^{+} \\
\bar{\nu}_{R}
\end{array}\right\rangle \oplus\left\langle d_{R}\right\rangle .
$$

In the Standard Model representation, we can think of the matter-antimatter distinction as a $\mathbb{Z}_{2}$-grading, because the Standard Model representation $F \oplus F^{*}$ splits into $F$ and $F^{*}$. By failing to respect this grading, the $\mathrm{SU}(5)$ symmetry group fails to preserve the usual distinction between matter and antimatter.

But the Standard Model has another $\mathbb{Z}_{2}$-grading that $\mathrm{SU}(5)$ does respect. This is the distinction between left- and right-handedness. Remember, the left-handed particles and antiparticles live in the even grades:

$$
\Lambda^{\mathrm{ev}} \mathbb{C}^{5} \cong\left\langle\bar{\nu}_{L}\right\rangle \oplus\left\langle e_{L}^{+}\right\rangle \oplus\left\langle\begin{array}{c}
u_{L} \\
d_{L}
\end{array}\right\rangle \oplus\left\langle\bar{u}_{L}\right\rangle \oplus\left\langle\begin{array}{c}
\nu_{L} \\
e_{L}^{-}
\end{array}\right\rangle \oplus\left\langle\bar{d}_{L}\right\rangle
$$

while the right-handed ones live in the odd grades:

$$
\Lambda^{\text {odd }} \mathbb{C}^{5} \cong\left\langle\nu_{R}\right\rangle \oplus\left\langle e_{R}^{-}\right\rangle \oplus\left\langle\begin{array}{c}
\bar{d}_{R} \\
\bar{u}_{R}
\end{array}\right\rangle \oplus\left\langle u_{R}\right\rangle \oplus\left\langle\begin{array}{c}
e_{R}^{+} \\
\bar{\nu}_{R}
\end{array}\right\rangle \oplus\left\langle d_{R}\right\rangle .
$$


The action of $\mathrm{SU}(5)$ automatically preserves this $\mathbb{Z}_{2}$-grading, because it comes from the $\mathbb{Z}$-grading on $\Lambda \mathbb{C}^{5}$, which $\mathrm{SU}(5)$ already respects.

This characteristic of the SU(5) theory lives on in its extension to Spin(10). There, the distinction between left and right is the only distinction among particles and antiparticles that $\operatorname{Spin}(10)$ knows about, because $\Lambda^{\mathrm{ev}} \mathbb{C}^{5}$ and $\Lambda^{\text {odd }} \mathbb{C}^{5}$ are irreducible. This says that the Spin(10) theory unifies all left-handed particles and antiparticles, and all right-handed particles and antiparticles.

In contrast, the $\operatorname{Spin}(4) \times \operatorname{Spin}(6)$ theory was all about adding a fourth "color", $w$, to represent leptons, and restoring a kind of symmetry between left and right by introducing a right-handed $\mathrm{SU}(2)$ that treats right-handed particles like the left-handed $\mathrm{SU}(2)$ treats left-handed particles.

Unlike the $\mathrm{SU}(5)$ theory, the $\operatorname{Spin}(4) \times \operatorname{Spin}(6)$ theory respects both $\mathbb{Z}_{2}$-gradings in the Standard Model: the matter-antimatter grading, and the right-left grading. The reason is that $\operatorname{Spin}(4) \times \operatorname{Spin}(6)$ respects the $\mathbb{Z}_{2} \times \mathbb{Z}_{2}$-grading on $\Lambda \mathbb{C}^{2} \otimes \Lambda \mathbb{C}^{3}$, and we have:

$$
\begin{aligned}
& F_{L} \cong \Lambda^{\text {odd }} \mathbb{C}^{2} \otimes \Lambda^{\text {odd }} \mathbb{C}^{3} \\
& F_{R} \cong \Lambda^{\text {ev }} \mathbb{C}^{2} \otimes \Lambda^{\text {odd }} \mathbb{C}^{3}, \\
& F_{L}^{*} \cong \Lambda^{\text {odd }} \mathbb{C}^{2} \otimes \Lambda^{\text {ev }} \mathbb{C}^{3} \\
& F_{R} \cong \Lambda^{\text {ev }} \mathbb{C}^{2} \otimes \Lambda^{\text {ev }} \mathbb{C}^{3}
\end{aligned}
$$

Moreover, the matter-antimatter grading and the right-left grading are all that $\operatorname{Spin}(4) \times \operatorname{Spin}(6)$ respects, since each of the four spaces listed is an irrep of this group.

When we extend $\operatorname{Spin}(4) \times \operatorname{Spin}(6)$ to the $\operatorname{Spin}(10)$ theory, we identify $\Lambda \mathbb{C}^{2} \otimes \Lambda \mathbb{C}^{3}$ with $\Lambda \mathbb{C}^{5}$. Then the $\mathbb{Z}_{2} \times \mathbb{Z}_{2}$-grading on $\Lambda \mathbb{C}^{2} \otimes \Lambda \mathbb{C}^{3}$ gives the $\mathbb{Z}_{2}$-grading on $\Lambda \mathbb{C}^{5}$ using addition in $\mathbb{Z}_{2}$. This sounds rather technical, but it is as simple as " "even + odd = odd":

$$
\Lambda^{\text {odd }} \mathbb{C}^{5} \cong\left(\Lambda^{\mathrm{ev}} \mathbb{C}^{2} \otimes \Lambda^{\text {odd }} \mathbb{C}^{3}\right) \oplus\left(\Lambda^{\text {odd }} \mathbb{C}^{2} \otimes \Lambda^{\mathrm{ev}} \mathbb{C}^{3}\right) \cong F_{R} \oplus F_{L}^{*}
$$

and "odd + odd = even", "even + even = even":

$$
\Lambda^{\mathrm{ev}} \mathbb{C}^{5} \cong\left(\Lambda^{\text {odd }} \mathbb{C}^{2} \otimes \Lambda^{\text {odd }} \mathbb{C}^{3}\right) \oplus\left(\Lambda^{\mathrm{ev}} \mathbb{C}^{2} \otimes \Lambda^{\mathrm{ev}} \mathbb{C}^{3}\right) \cong F_{L} \oplus F_{R}^{*}
$$

Recall that $F_{R} \oplus F_{L}^{*}$ consists of all the fermions and antifermions that are righthanded, while $F_{L} \oplus F_{R}^{*}$ consists of the left-handed ones.

Furthermore, the two routes to the Spin(10) theory that we have described, one going through $\mathrm{SU}(5)$ and the other through $\operatorname{Spin}(4) \times \operatorname{Spin}(6)$, are compatible. In 
other words, this cube commutes:

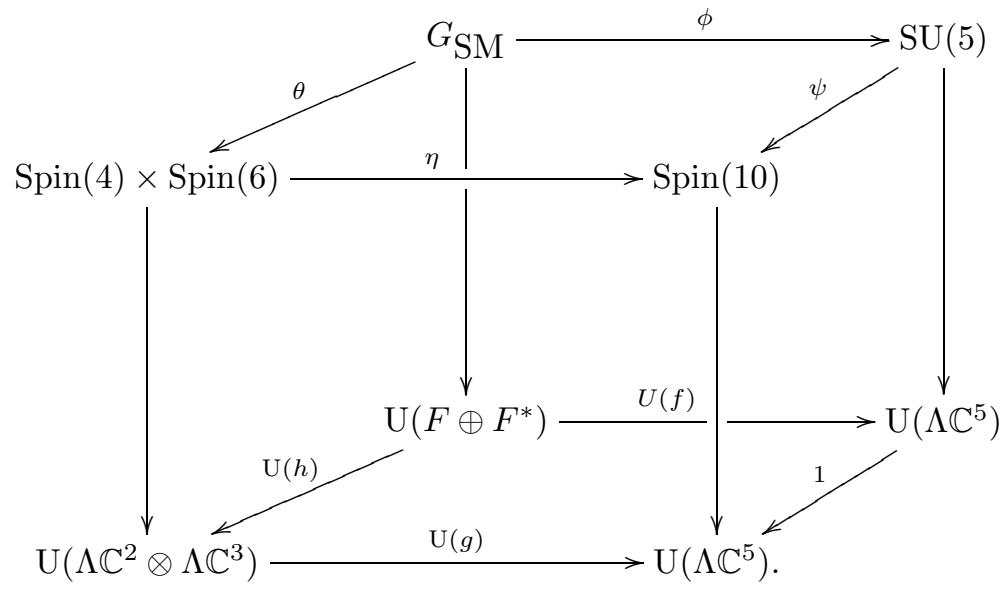

So, all four theories fit together in an elegant algebraic pattern. What this means for physics, if anything, remains unknown. Yet we cannot resist feeling that it means something, and we cannot resist venturing a guess: the Standard Model is exactly the theory that reconciles the visions built into the $\mathrm{SU}(5)$ and $\operatorname{Spin}(4) \times \operatorname{Spin}(6)$ theories.

What this might mean is not yet precise, but since all these theories involve symmetries and representations, the "reconciliation" must take place at both those levels, and we can see this in a precise way. First, at the level of symmetries, our Lie groups are related by the commutative square of homomorphisms:

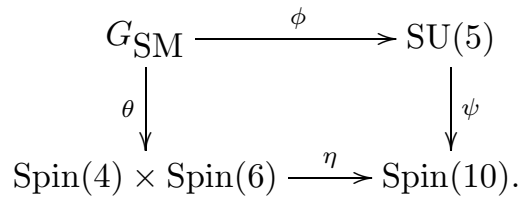

Because this commutes, the image of $G_{\mathrm{SM}}$ lies in the intersection of the images of $\operatorname{Spin}(4) \times \operatorname{Spin}(6)$ and $\mathrm{SU}(5)$ inside $\operatorname{Spin}(10)$. But we claim it is precisely that intersection!

To see this, first recall that the image of a group under a homomorphism is just the quotient group formed by modding out the kernel of that homomorphism. If we do this for each of our homomorphisms above, we get a commutative square of inclusions:

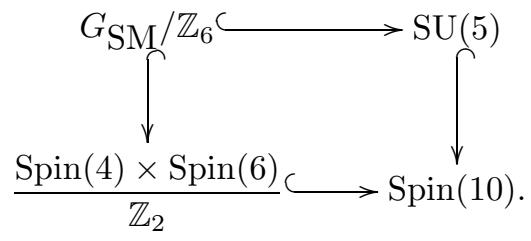

This implies that

$$
G_{\mathrm{SM}} / \mathbb{Z}_{6} \subseteq \mathrm{SU}(5) \cap\left(\frac{\operatorname{Spin}(4) \times \operatorname{Spin}(6)}{\mathbb{Z}_{2}}\right)
$$


as subgroups of Spin(10). To make good on our claim, we must show that these subgroups are equal:

$$
G_{\mathrm{SM}} / \mathbb{Z}_{6}=\mathrm{SU}(5) \cap\left(\frac{\operatorname{Spin}(4) \times \operatorname{Spin}(6)}{\mathbb{Z}_{2}}\right) .
$$

In other words, our commutative square of inclusions is a "pullback square".

As a step towards showing this, first consider what happens when we pass from the spin groups to the rotation groups. We can accomplish this by modding out by an additional $\mathbb{Z}_{2}$ above. We get another commutative square of inclusions:

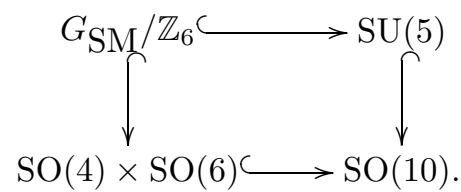

Here the reader may wonder why we could quotient $(\operatorname{Spin}(4) \times \operatorname{Spin}(6)) / \mathbb{Z}_{2}$ and $\operatorname{Spin}(10)$ by $\mathbb{Z}_{2}$ without having to do the same for their respective subgroups, $G_{\mathrm{SM}} / \mathbb{Z}_{6}$ and SU(5). It is because $\mathbb{Z}_{2}$ intersects both of those subgroups trivially. We can see this for $\mathrm{SU}(5)$ because we know the inclusion $\mathrm{SU}(5) \hookrightarrow \operatorname{Spin}(10)$ is just the lift of the inclusion $\mathrm{SU}(5) \hookrightarrow \mathrm{SO}(10)$ to universal covers, so it makes this diagram commute:

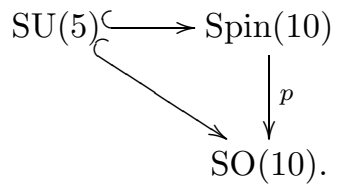

But this means that $\mathrm{SU}(5)$ intersects $\mathbb{Z}_{2}=\operatorname{ker} p$ in only the identity. The subgroup $G_{\mathrm{SM}} / \mathbb{Z}_{6}$ therefore intersects $\mathbb{Z}_{2}$ trivially as well.

Now, let us show:

Theorem 8. $G_{\mathrm{SM}} / \mathbb{Z}_{6}=\mathrm{SU}(5) \cap(\mathrm{SO}(4) \times \mathrm{SO}(6)) \subseteq \mathrm{SO}(10)$.

Proof. We can prove this in the same manner that we showed, in Section 3.1, that

$$
G_{\mathrm{SM}} / \mathbb{Z}_{6} \cong \mathrm{S}(\mathrm{U}(2) \times \mathrm{U}(3)) \subseteq \mathrm{SU}(5)
$$

is precisely the subgroup of $\mathrm{SU}(5)$ that preserves the $2+3$ splitting of $\mathbb{C}^{5} \cong \mathbb{C}^{2} \oplus \mathbb{C}^{3}$.

To begin with, the group $\mathrm{SO}(10)$ is the group of orientation-preserving symmetries of the ten-dimensional real inner product space $\mathbb{R}^{10}$. But $\mathbb{R}^{10}$ is suspiciously like $\mathbb{C}^{5}$, a five-dimensional complex inner product space. Indeed, if we forget the complex structure on $\mathbb{C}^{5}$, we get an isomorphism $\mathbb{C}^{5} \cong \mathbb{R}^{10}$, a real inner product space with symmetries $\mathrm{SO}(10)$. We can consider the subgroup of $\mathrm{SO}(10)$ that preserves the original complex structure. This is $\mathrm{U}(5) \subseteq \mathrm{SO}(10)$. If we further pick a volume form on $\mathbb{C}^{5}$, i.e., a nonzero element of $\Lambda^{5} \mathbb{C}^{5}$, and look at the symmetries fixing that volume form, we get a copy of $\mathrm{SU}(5) \subseteq \mathrm{SO}(10)$.

Then we can pick a $2+3$ splitting on $\mathbb{C}^{5} \cong \mathbb{C}^{2} \oplus \mathbb{C}^{3}$. The subgroup of $\mathrm{SU}(5)$ that also preserves this is

$$
\mathrm{S}(\mathrm{U}(2) \times \mathrm{U}(3)) \hookrightarrow \mathrm{SU}(5) \hookrightarrow \mathrm{SO}(10) .
$$


These inclusions form the top and right sides of our square:

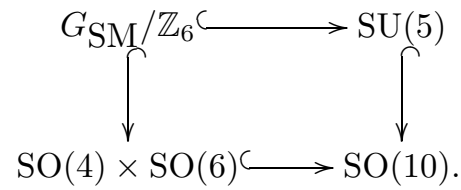

We can also reverse the order of these processes. Imposing a $2+3$ splitting on $\mathbb{C}^{5}$ yields a $4+6$ splitting on the underlying real vector space, $\mathbb{R}^{10} \cong \mathbb{R}^{4} \oplus \mathbb{R}^{6}$. The subgroup of $\mathrm{SO}(10)$ that preserves this splitting is $\mathrm{S}(\mathrm{O}(4) \times \mathrm{O}(6))$ : the block diagonal matrices with $4 \times 4$ and $6 \times 6$ orthogonal blocks and overall determinant 1. The connected component of this subgroup is $\mathrm{SO}(4) \times \mathrm{SO}(6)$.

The direct summands in $\mathbb{R}^{4} \oplus \mathbb{R}^{6}$ came from forgetting the complex structure on $\mathbb{C}^{2} \oplus \mathbb{C}^{3}$. The subgroup of $\mathrm{S}(\mathrm{O}(4) \times \mathrm{O}(6))$ preserving the original complex structure is $\mathrm{U}(2) \times \mathrm{U}(3)$, and the subgroup of this that also fixes a volume form on $\mathbb{C}^{5}$ is $\mathrm{S}(\mathrm{U}(2) \times \mathrm{U}(3))$. This group is connected, so it must lie entirely in the connected component of the identity, and we get the inclusions:

$$
\mathrm{S}(\mathrm{U}(2) \times \mathrm{U}(3)) \hookrightarrow \mathrm{SO}(4) \times \mathrm{SO}(6) \hookrightarrow \mathrm{SO}(10) .
$$

These maps form the left and bottom sides of our square.

It follows that $G_{\mathrm{SM}} / \mathbb{Z}_{6}$ is precisely the subgroup of $\mathrm{SO}(10)$ that preserves a complex structure on $\mathbb{R}^{10}$, a chosen volume form on the resulting complex vector space, and a $2+3$ splitting on this space. But this $2+3$ splitting is the same as a compatible $4+6$ splitting of $\mathbb{R}^{10}$, one in which each summand is a complex vector subspace as well as a real subspace. This means that

$$
G_{\mathrm{SM}} / \mathbb{Z}_{6}=\mathrm{SU}(5) \cap \mathrm{S}(\mathrm{O}(4) \times \mathrm{O}(6)) \subseteq \mathrm{SO}(10),
$$

and since $G_{\mathrm{SM}} / \mathbb{Z}_{6}$ is connected,

$$
G_{\mathrm{SM}} / \mathbb{Z}_{6}=\mathrm{SU}(5) \cap(\mathrm{SO}(4) \times \mathrm{SO}(6)) \subseteq \mathrm{SO}(10),
$$

as desired.

From this, a little diagram chase proves our earlier claim:

Theorem 9. $G_{\mathrm{SM}} / \mathbb{Z}_{6}=\mathrm{SU}(5) \cap(\operatorname{Spin}(4) \times \operatorname{Spin}(6)) / \mathbb{Z}_{2} \subseteq \operatorname{Spin}(10)$.

Proof. By now we have built the following commutative diagram:

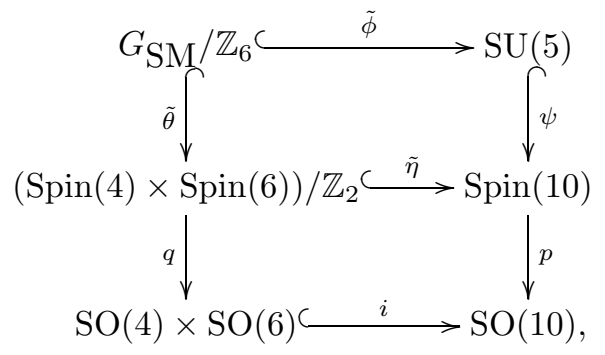


where both of the bottom vertical arrows are two-to-one, but the composite vertical maps $q \tilde{\theta}$ and $p \psi$ are one-to-one. Our previous theorem says that the big square with $q \tilde{\theta}$ and $p \psi$ as vertical sides is a pullback. Now we must show that the upper square is also a pullback. So, suppose we are given $g \in(\operatorname{Spin}(4) \times \operatorname{Spin}(6)) / \mathbb{Z}_{2}$ and $g^{\prime} \in \mathrm{SU}(5)$ with

$$
\tilde{\eta}(g)=\psi\left(g^{\prime}\right)
$$

We need to show that there exists $x \in G_{\mathrm{SM}} / \mathbb{Z}_{6}$ such that

$$
\tilde{\theta}(x)=g, \quad \tilde{\phi}(x)=g^{\prime} .
$$

Now, we know that

$$
i q(g)=p \tilde{\eta}(g)=p \psi\left(g^{\prime}\right),
$$

so since the big square is a pullback, there exists $x \in G_{\mathrm{SM}} / \mathbb{Z}_{6}$ with

$$
q \tilde{\theta}(x)=q(g), \quad \tilde{\phi}(x)=g^{\prime} .
$$

The second equation is half of what we need to show. So, we only need to check that the first equation implies $\tilde{\theta}(x)=g$.

The kernel of $q$ consists of two elements, which we will simply call \pm 1 . Since $q \tilde{\theta}(x)=q(g)$, we know that

$$
\pm \tilde{\theta}(x)=g
$$

Since $\tilde{\eta}(g)=\psi\left(g^{\prime}\right)$, we thus have

$$
\tilde{\eta}( \pm \tilde{\theta}(x))=\psi\left(g^{\prime}\right)=\psi \tilde{\phi}(x) .
$$

The one-to-one map $\tilde{\eta}$ sends the kernel of $q$ to the kernel of $p$, which consists of two elements that we may again call \pm 1 . So, $\pm \tilde{\eta} \tilde{\theta}(x)=\psi \tilde{\phi}(x)$. On the other hand, since the top square commutes we know that $\tilde{\eta} \tilde{\theta}(x)=\psi \tilde{\phi}(x)$. Thus the element \pm 1 must actually be 1 , so $g=\tilde{\theta}(x)$ as desired.

In short, the Standard Model has precisely the symmetries shared by both the $\mathrm{SU}(5)$ theory and the $\operatorname{Spin}(4) \times \operatorname{Spin}(6)$ theory. Now let us see what this means for the Standard Model representation.

We can "break the symmetry" of the Spin(10) theory in two different ways. In the first way, we start by picking the subgroup of $\operatorname{Spin}(10)$ that preserves the $\mathbb{Z}$ grading and volume form in $\Lambda \mathbb{C}^{5}$. This is $\mathrm{SU}(5)$. Then we pick the subgroup of $\mathrm{SU}(5)$ that respects the splitting of $\mathbb{C}^{5}$ into $\mathbb{C}^{2} \oplus \mathbb{C}^{3}$. This subgroup is the Standard Model gauge group, modulo a discrete subgroup, and its representation on $\Lambda \mathbb{C}^{5}$ is the Standard Model representation.

We can draw this symmetry breaking process in the following diagram:

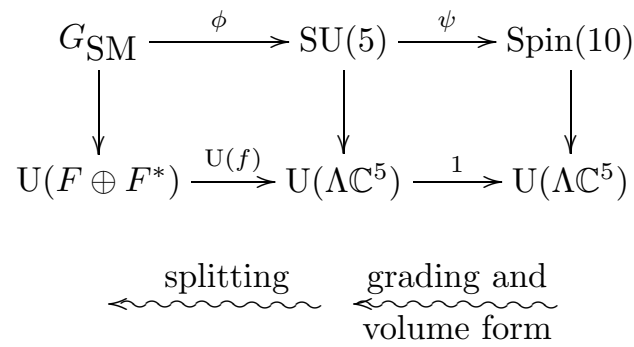

The SU(5) theory shows up as a "halfway house" here. 
We can also break the symmetry of $\operatorname{Spin}(10)$ in a way that uses the $\operatorname{Spin}(4) \times$ Spin(6) theory as a halfway house. We do essentially the same two steps as before, but in the reverse order! This time we start by picking the subgroup of $\operatorname{Spin}(10)$ that respects the splitting of $\mathbb{R}^{10}$ as $\mathbb{R}^{4} \oplus \mathbb{R}^{6}$. This subgroup is $\operatorname{Spin}(4) \times \operatorname{Spin}(6)$ modulo a discrete subgroup. The two factors in this subgroup act separately on the factors of $\Lambda \mathbb{C}^{5} \cong \Lambda \mathbb{C}^{2} \otimes \Lambda \mathbb{C}^{3}$. Then we pick the subgroup of $\operatorname{Spin}(4) \times \operatorname{Spin}(6)$ that respects the $\mathbb{Z}$-grading and volume form on $\Lambda \mathbb{C}^{5}$. This subgroup is the Standard Model gauge group, modulo a discrete subgroup, and its representation on $\Lambda \mathbb{C}^{2} \otimes \Lambda \mathbb{C}^{3}$ is the Standard Model representation.

We can draw this alternate symmetry breaking process in the following diagram:

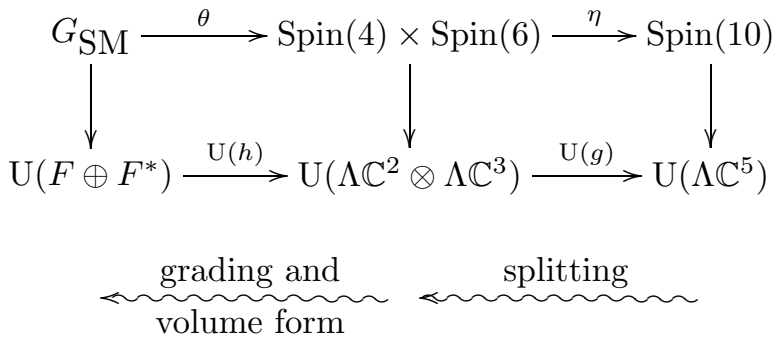

Will these tantalizing patterns help us understand physics beyond the Standard Model? Only time will tell.

\section{ACKNOWLEDGMENTS}

We thank the denizens of the $n$-Category Café for catching mistakes and suggesting stylistic improvements. This research was supported by a grant from the Foundational Questions Institute.

\section{ABOut THE AUTHORS}

John Baez is a mathematician at the University of California, Riverside, and John Huerta is a graduate student there, working on algebra inspired by particle physics and string theory.

\section{REFERENCES}

[1] J. F. Adams, Lectures on Exceptional Lie Groups, Zafer Mahmoud and Mamoru Mimura, eds., University of Chicago Press, Chicago, 1996. MR1428422 (98b:22001)

[2] M. F. Atiyah, R. Bott and A. Shapiro, Clifford modules, Topology 3 (1964), 3-38. MR0167985 $(29: 5250)$

[3] John C. Baez and Javier P. Muniain, Gauge Fields, Knots and Gravity, World Scientific, Singapore, 1994. MR1313910 (95k:83001)

[4] Stefano Bertolini, Luca Di Luzio and Michal Malinsky, Intermediate mass scales in the nonsupersymmetric $\mathrm{SO}(10)$ grand unification: a reappraisal, available at arXiv:0903.4049

[5] Lowell Brown, Quantum Field Theory, Cambridge Univ. Press, Cambridge, 1994. MR.1231339 (94h:81002)

[6] Claude Chevalley, The Algebraic Theory of Spinors and Clifford Algebras, Springer, Berlin, 1996. MR1636473 (99f:01028)

[7] Benedict Cassen and Edward U. Condon, On Nuclear Forces, Phys. Rev. 50 (1936), 846, reprinted in D. M. Brink, Nuclear Forces, Pergamon, Oxford, 1965, pp. 193-201.

[8] Robert P. Crease and Charles C. Mann, The Second Creation: Makers of the Revolution in Twentieth-Century Physics, Rutgers University Press, New Brunswick, New Jersey, 1996. MR:1677561 (2000f:81002) 
[9] Andrzej Derdzinski, Geometry of the Standard Model of Elementary Particles, Springer, Berlin, 1992. MR.1219312 (95f:81101)

[10] Howard Georgi, The state of the art - gauge theories, in Particles and Fields - 1974, ed. Carl E. Carlson, AIP Conference Proceedings 23, 1975, pp. 575-582.

[11] Howard Georgi, Lie Algebras in Particle Physics: From Isospin to Unified Theories, Westview Press, Boulder, Colorado, 1999. MR644800 (83e:81089)

[12] Howard Georgi and Sheldon Glashow, Unity of all elementary-particle forces, Phys. Rev. Lett. 32(8) Feb 1974, 438-441.

[13] David Griffiths, Introduction to Elementary Particles, Wiley, New York 1987.

[14] Brian Hall, Lie Groups, Lie Algebras, and Representations, Springer, Berlin, 2003. MR1997306 (2004i:22001)

[15] Werner Heisenberg, Zeitschr. f. Phys. 77 (1932), 1; English translation in D. M. Brink, Nuclear Forces, Pergamon, Oxford, 1965, pp. 144-154.

[16] Laurie Brown, Max Dresden, Lillian Hoddeson and Michael Riordan, eds., The Rise of the Standard Model, Cambridge Univ. Press, Cambridge, 1997. MR1601138 (98h:81002)

[17] Kerson Huang, Quarks, Leptons 83 Gauge Fields, World Scientific, Singapore, 1992. MR704019 (84k:81138)

[18] Chris Isham, Modern Differential Geometry for Physicists, World Scientific, Singapore, 1999. MR.1698234 (2000e:53001)

[19] T. D. Lee, Particle Physics and Introduction to Field Theory, Harwood, 1981. MR633154 (83d:81001)

[20] Harry J. Lipkin, Lie Groups for Pedestrians, Dover, Mineola, New York, 2002. MR1918338

[21] R. N. Mohapatra, Unification and Supersymmetry: The Frontiers of Quark-Lepton Physics, Springer, 1992. MR854671 (87j:81276)

[22] Gregory L. Naber, Topology, Geometry and Gauge Fields: Foundations, Springer, Berlin, 1997. MR1444352 (99b:58001)

[23] Gregory L. Naber, Topology, Geometry and Gauge Fields: Interactions, Springer, Berlin, 2000. MR1744816 (2001g:53058)

[24] Mikio Nakahara, Geometry, Topology, and Physics, Academic Press, 1983.

[25] Abraham Pais, Inward Bound: Of Matter and Forces in the Physical World, Oxford University Press, 1988.

[26] Jogesh C. Pati, Proton decay: A must for theory, a challenge for experiment, available at arXiv:hep-ph/0005095

[27] Jogesh C. Pati, Probing grand unification through neutrino oscillations, leptogenesis, and proton decay, Int. J. Mod. Phys. A 18 (2003), 4135-4156. Also available at arXiv:hep-ph/0305221

[28] Jogesh C. Pati and Abdus Salam, Lepton number as the fourth "color", Phys. Rev. D 10 (1974), 275-289.

[29] Michael E. Peskin, Beyond the Standard Model, available at arXiv:hep-ph/970549

[30] Michael E. Peskin and Dan V. Schroeder, An Introduction to Quantum Field Theory, Westview Press, 1995. MR1402248 (97j:81001)

[31] Graham G. Ross, Grand Unified Theories, Benjamin/Cummings, $1985 . \quad$ MR.783830 (86c:81097)

[32] Lewis H. Ryder, Quantum Field Theory, Cambridge Univ. Press, Cambridge, 1996. MR:1404519 (97h:81001)

[33] Emilio Segrè, From X-Rays to Quarks: Modern Physicists and Their Discoveries, W.H. Freeman, San Francisco, 1980.

[34] Shlomo Sternberg, Group Theory and Physics, Cambridge Univ. Press, Cambridge, 1995. MR.1287387 (95i:20001)

[35] Mark Srednicki, Quantum Field Theory, Cambridge Univ. Press, 2007. Also available at http://www.physics.ucsb.edu/ mark/qft.html

[36] Anthony Sudbery, Quantum Mechanics and the Particles of Nature: An Outline for Mathematicians, Cambridge Univ. Press, Cambridge, 1986. MR.2245407

[37] Robin Ticciati, Quantum Field Theory for Mathematicians, Cambridge Univ. Press, 1999. MR 1699269 (2000h:81002)

[38] Michael Tinkham, Group Theory and Quantum Mechanics, Dover, Mineola, New York, 2003. 
[39] Edward Witten, Grand unification with and without supersymmetry, in Introduction to supersymmetry in particle and nuclear physics, O. Castanos, A. Frank, L. Urrutia, eds., Plenum Press, 1984, pp. 53-76. MR758626

[40] Anthony Zee, Quantum Field Theory in a Nutshell, Princeton Univ. Press, Princeton, 2003. MR1978227 (2004i:81003)

Department of Mathematics, University of California, Riverside, California 92521

E-mail address: baez@math.ucr.edu

Department of Mathematics, University of California, Riverside, California 92521

E-mail address: huerta@math.ucr.edu 